\title{
A Maximum Principle for Smooth Infinite Horizon Optimal Control Problems with State Constraints and with Terminal Constraints at Infinity
}

\author{
Atle Seierstad \\ University of Oslo, Oslo, Norway \\ Email: atle.seierstad@econ.uio.no \\ Received 14 May 2015; accepted 26 September 2015; published 29 September 2015 \\ Copyright (C) 2015 by author and Scientific Research Publishing Inc. \\ This work is licensed under the Creative Commons Attribution International License (CC BY). \\ http://creativecommons.org/licenses/by/4.0/

\begin{abstract}
Necessary conditions for optimality are proved for smooth infinite horizon optimal control problems with unilateral state constraints (pathwise constraints) and with terminal conditions on the states at the infinite horizon. The aim of the paper is to obtain strong necessary conditions including transversality conditions at infinity, which in many cases lead to a set of candidates for optimality containing only a few elements, similar to what is the case in finite horizon problems. However, strong growth conditions are needed for the results to hold.
\end{abstract}

\section{Keywords}

Infinite Horizon, Optimal Control, State Constraints

\section{Introduction}

The aim of this paper is, in a control problem with unilateral state constraints and terminal conditions at infinity, to obtain necessary conditions, with a full set of transversality conditions at infinity, which frequently make it possible to narrow down the set of candidates for optimality to only a few, or sometimes a single one. In infinite horizon problems without unilateral state constraints (pathwise constraints), with or without terminal conditions on the states at the infinite horizon, there exist various types of necessary conditions for optimality, and examples are [1] (without a transversality condition), and a number of results with certain limited types of transversality conditions, for example [2], slightly generalized in [3]. See the latter paper and [4] for several further references (see also [5]). The limited types of transversality conditions mentioned are in problems with several 
states-often insufficient if one wishes to avoid getting an infinite number of candidates. With strong growth conditions there exist necessary conditions, with a full set of transversality conditions at infinity, which in many cases make it possible to narrow down the set of candidates to only a few, or sometimes a single one, see Theorem 16, p. $244^{1}$ in [5]. For nonsmooth problems with a full set of transversality conditions in the infinite horizon case, see [6]. For such problems, see also [7].

The novelty of the results in this paper is hence the establishment of necessary conditions that include a full set of transversality conditions at infinity in an infinite horizon problem with both terminal constraints at the infinite horizon and unilateral state constraints (constraints of the form $g^{j}(t, x) \geq 0$ for all $t$ ). Strong growth conditions are needed for the results to hold.

For Michel-type necessary condition in the case of unilateral state constraints, sees [8].

The growth conditions used below, ((11), (12), (13)) are more demanding than the conditions applied in [9] for the case of no unilateral state constraints and no terminal constraints (problems with a dominant discount). In later work the authors use even more general conditions, see [10] (see also [11], and [12] for problems with a special structure).

The results below are of especial interest in the case where not all states are completely constrained at infinity. In the opposite case, generalizations of Halkin's infinite horizon theorem in [1] to problems with unilateral state constraints where no transversality conditions appear, like Theorem 9, p. 381 in [6], frequently yield enough information for determining one or a few candidates for optimality. When not all states are completely constrained at infinity, transversality conditions related to the terminal conditions are needed, unless one can accept the possibility of an infinite number of candidates for optimality.

In certain cases there is a danger of degeneracy of multipliers. See the early review in [13] and [14]. We have added conditions that secure nondegeneracy of multipliers in some such cases, in particular in the case where unilateral constraints are satisfied as equalities by the initial state (the state at time zero). See [15]-[17] for a presentation of similar conditions in the finite horizon case, as well as for a number of references for this case (see for example [18]-[22]).

\section{The Control Problem, Necessary Conditions, and Examples}

Consider the problem

$$
\max \int_{0}^{\infty} f^{0}(t, x, u) \mathrm{d} t
$$

where $x=\left(x^{1}, \cdots, x^{n}\right)$, subject to

$$
\begin{gathered}
\dot{x}=f(t, x, u), u \in U, x(0)=x_{0} \in \mathbb{R}^{n} \\
g^{j}(t, x) \geq 0 \text { for } t \in\left[a_{j}, b_{j}\right]=: J^{j}, j=1, \cdots, j^{*} \\
x^{i}(\infty)=\bar{x}^{i}, i \in\left\{1, \cdots, n^{\prime}\right\}, x^{i}(\infty) \geq \bar{x}^{i}, i \in\left\{n^{\prime}+1, \cdots, n^{\prime \prime}\right\}, n^{\prime \prime} \leq n
\end{gathered}
$$

where we require that $\lim _{t \rightarrow \infty} x^{i}(t):=x^{i}(\infty)$ exists for $i \leq n^{\prime \prime}$ and where $f=\left(f^{1}, \cdots, f^{n}\right)$. Here, $j^{*}, n^{\prime}$, $n^{\prime \prime}$ and $n$ are given natural numbers, and we allow for the case where there are no equality constraints or no inequality constraints in (4) (in which cases $n^{\prime}=0$ and $n^{\prime \prime}=n^{\prime}$, so $\left\{1, \cdots, n^{\prime}\right\}$ and/or $\left\{n^{\prime}+1, \cdots, n^{\prime \prime}\right\}$ are empty sets). Furthermore, $a_{j}<b_{j}, U \subset \mathbb{R}^{\tilde{k}}, x_{0}, \bar{x}^{i}, f^{i}, g^{j}$, are fixed entities, $u$ the control. It is possible that $b_{j}=\infty$, in which case $\left[a_{j}, b_{j}\right]$ is replaced by $\left[a_{j}, \infty\right)$. We want to maximize the objective in (1) over the set $\mathcal{U}$ of all measurable functions $u($.$) taking values in U$ and being bounded on bounded time intervals, subject to (2)-(4). When the solution $x($.$) corresponding to such a u($.$) satisfies (2)-(4), we call (x(),. u()$,$) ad-$ missible. Below $\left(x^{*}(),. u^{*}().\right)$ will be a given optimal admissible pair, assumed to exist.

We assume that $g^{j}$ is continuous in $(t, x) \in[0, \infty) \times \mathbb{R}^{n}$, that $f^{i}$ is measurable in $t \in[0, \infty)$, continuous in $(x, u) \in \mathbb{R}^{n} \times U$, with derivatives $g_{x}^{j}$ and $f_{x}^{i}$, where $g_{x}^{j}$ is continuous in $x$ and $f_{x}^{i}$ is continuous in $(x, u)$. We also assume, for any bounded sets $J^{\prime} \subset J:=[0, \infty)$ and $U^{\prime} \subset U$, that $\sup _{t \in J^{\prime}}\left|g_{x}^{j}\left(t, x^{*}(t)\right)\right|<\infty$, and that for any $x, \sup _{t \in J^{\prime}, u \in U^{\prime}}\left|\left(f^{0}(t, x, u), f(t, x, u)\right)\right|<\infty$. These assumptions are called the basic smoothness assumptions. At various points some strengthening of these assumptions are added.

\footnotetext{
${ }^{1}$ In that theorem, correct the inequality $b>k$ by replacing it by $b>(n-m) k$.
} 
The following definitions are needed: let $f^{*}(t):=f\left(t, x^{*}(t), u^{*}(t)\right), \quad f_{x}^{*}(t):=f_{x}\left(t, x^{*}(t), u^{*}(t)\right)$, $f^{0 *}(t):=f^{0}\left(t, x^{*}(t), u^{*}(t)\right), \quad f_{x}^{0 *}(t):=f_{x}^{0}\left(t, x^{*}(t), u^{*}(t)\right)$, let $C(t, s)$ be the resolvent of the equation $\dot{q}=f_{x}^{*}(t) q\left(C(s, s)=I, \quad I\right.$ the identity map), $C(t, s)=0$ if $t<s, \quad F(t):=f\left(t, x^{*}(t), U\right)$, $G_{\infty}:=\left\{j: b_{j}=\infty\right\}, \quad G^{\infty}:=\left\{j \in G_{\infty}: \liminf _{t \rightarrow \infty} g^{j}\left(t, x^{*}(t)\right)>0\right\}, \quad G(\infty):=G_{\infty} \backslash G^{\infty}$.

In Theorem 1, in addition to the basic assumptions, assumptions (5)-(15) below are needed. It is assumed that for all $j \in G(\infty)$

$$
\lim _{t \rightarrow \infty} g^{j}\left(t, x^{*}(t)\right)=: \hat{g}^{j}(\infty) \text { and } \lim _{t \rightarrow \infty} g_{x}^{j}\left(t, x^{*}(t)\right)=: \hat{g}_{x}^{j}(\infty) \text { exist }
$$

We shall make use of some constraint qualifications, (6) and (8) below, related to $g^{j} \geq 0$. Define $k^{\infty}:=\left\{k \in\left\{n^{\prime}+1, \cdots, n^{\prime \prime}\right\}: x^{* k}(\infty)=\bar{x}^{k}\right\}, \quad C^{\infty}:=\left\{c \in \mathbb{R}^{n}: c^{k}=0\right.$ for $k \leq n^{\prime}, c^{k} \geq 0$ for $\left.k \in k^{\infty}\right\}$, $J^{*}(j, \beta):=\left\{t \in J^{j}: g^{j}\left(t, x^{*}(t)\right) \leq \beta\right\}, \quad G_{t}:=\left\{j: t \in J^{j}\right\}, \quad G(t):=\left\{j \in G_{t}: g^{j}\left(t, x^{*}(t)\right)=0\right\}$, $G(t+)=\left\{j \in G(t):\right.$ for some $s>t$ arbitrarily near $\left.t, g^{j}\left(s, x^{*}(s)\right)=0\right\}$

$$
\text { For some } c^{*} \in C^{\infty} \text {, for all } j \in G(\infty), \hat{g}_{x}^{j}(\infty) c^{*}>0
$$

((6) holds vacuously if $G(\infty)=\varnothing)$.

$$
g^{j} \text { is } C^{1} \text { for } j=1, \cdots, j^{*}
$$

Either $G(0+)=\varnothing$, or $G(0+) \neq \varnothing$ and for all $j \in G(0+)$, (9) and (10) below hold

$$
\begin{gathered}
t \rightarrow\left(f^{0}\left(t, x^{*}(t), u\right), f\left(t, x^{*}(t), u\right)\right) \text { is, for all } u \text {, right continuous } \\
\text { and for some } v^{*} \text { in } \operatorname{co} F(0) \\
\text { for any } j \in G(0+), g_{x}^{j}\left(0, x^{*}(0)\right) v^{*}+g_{t}^{j}\left(0, x^{*}(0)\right)>0
\end{gathered}
$$

Either $^{2}$

$(\alpha) G(0+)$ contains only one index $j$, or

$(\beta) 0$ is a right Lebesgue point of $\left(f^{0}\left(., x_{0}, u^{*}().\right), f\left(., x_{0}, u^{*}().\right)\right)$, or

$(\gamma)$ For any $u,\left(f^{0}, f\right)$ is continuous in $(t, x)$

$$
\text { and differentiable in }(t, x) \text { at }\left(t, x^{*}(t)\right) \text { for all } t
$$

The following growth conditions are also needed: For some $n^{1} \geq n^{\prime \prime}$

$$
\int_{0}^{\infty}\left|f^{i}\left(t, x^{*}(t), u^{*}(t)\right)\right| \mathrm{d} t<\infty, i=0, \cdots, n^{1} \leq n
$$

and there exist some positive constants $A, B, C, a, b, \kappa$ such that

$$
\begin{gathered}
\text { for } i=0, \cdots, n^{1} \text {, for all }(t, x),\left|f_{x^{j}}^{i}\left(t, x, u^{*}(t)\right)\right| \leq\left\{\begin{array}{l}
A \mathrm{e}^{-a t} \text { for } j \leq n^{1} \\
B \mathrm{e}^{-b t} \text { for } j>n^{1}
\end{array}\right. \\
\text { for } i>n^{1} \text {, for all }(t, x),\left|f_{x^{j}}^{i}\left(t, x, u^{*}(t)\right)\right| \leq \begin{cases}C \mathrm{e}^{\kappa t} & \text { for } j \leq n^{1} \\
\kappa & \text { for } j>n^{1}\end{cases}
\end{gathered}
$$

where $b>\kappa\left(n-n^{1}\right)$. In (10) ( $\gamma$ ), we also need that for some $\tilde{k}>0, \quad\left|f_{t}^{i}\left(t, x, u^{*}(t)\right)\right| \leq A$ for all $(t, x), \quad t \leq \tilde{k}$. Assume finally that, for all $j$

\footnotetext{
${ }^{2} \mathrm{~A}$ right Lebesgue point $s$ of any integrable function $a(t):[0, \infty) \rightarrow \mathbb{R}^{n}$ means a point $s$ such that $\lim _{d \downarrow 0}(1 / d) \int_{s}^{s+d}|a(t)-a(s)| \mathrm{d} t=0$. If $v^{*}$ in (9) even belongs to $F(0)$ and (10) ( $\beta$ ) holds, then the right continuity in (9) can be weakened to 0 being a right Lebesgue point of $t \rightarrow f\left(t, x_{0}, u\right)$ for all $u \in U$.
} 


$$
g^{j} \text { depends only on } x^{i}, i \leq n^{1}
$$

For $j \in G_{\infty}, z \rightarrow g_{x}^{j}\left(t, x^{*}(t)+z\right)$ is continuous, uniformly in $t, \sup _{t \in[0, \infty)}\left|g_{x}^{j}\left(t, x^{*}(t)\right)\right|<\infty$

Define $H\left(t, x, u, p_{0}, p\right)=p_{0} f^{0}+p f=p_{0} f^{0}+\sum_{i=1}^{n} p^{i} f^{i}$. The following necessary conditions for optimality holds.

Theorem 1. (Necessary condition, infinite horizon) Assume (5)-(8), (11)-(15) and the basic smoothness as sumptions. There exist a number $p_{0} \in\{0,1\}$, vectors $\hat{p}_{\infty}=\left(\hat{p}_{\infty}^{1}, \cdots, \hat{p}_{\infty}^{n}\right), \quad p_{\infty}:=\left(p_{\infty}^{1}, \cdots, p_{\infty}^{n}\right)$, bounded vector functions $p(t):=\left(p^{1}(t), \cdots, p^{n}(t)\right)$ and $v(t):=\left(v^{1}(t), \cdots, v^{j^{*}}(t)\right), \quad v^{j}$ nondecreasing and right continuous on $(0, \infty)$, such that if $p(t, T)^{3}$ satisfies, for $t \in[0, T]$, the equation

$$
p(t, T)=p_{\infty}+\hat{p}_{\infty}+\int_{t}^{T} H_{x}\left(r, x^{*}(r), u^{*}(r), p_{0}, p(r, T)\right) \mathrm{d} r+\sum_{j} \int_{[t, T)} g_{x}^{j}\left(r, x^{*}(r)\right) \mathrm{d} v^{j}(r)
$$

then $p(t)=\lim _{T \rightarrow \infty} p(t, T)$ (the limit does exist), $\lim _{t \rightarrow \infty} p(t)=p_{\infty}+\hat{p}_{\infty}, \quad p(t)$ is left continuous on $(0, \infty)$ and satisfies (16) for $T=\infty$ (the integrals exist), and

$$
H\left(t, x^{*}(t), u, p_{0}, p(t)\right) \leq H\left(t, x^{*}(t), u^{*}(t), p_{0}, p(t)\right) \text { for all } u \in U \text {, for a.e. } t
$$

Moreover,

$$
\begin{gathered}
\left(p_{0}, p_{\infty}+\hat{p}_{\infty}, \sum_{j} \int_{(0, \infty)} \mathrm{d} v^{j}(t)\right) \neq 0 \\
v^{j}(t) \text { is constant on }\left[0, a_{j}\right) \text { and }\left(b_{j}, \infty\right) \text { and on intervals on which } g^{j}\left(t, x^{*}(t)\right)>0 \\
p_{\infty}^{i} \geq 0 \text { for } i \in\left\{n^{\prime}+1, \cdots, n^{\prime \prime}\right\} \\
p_{\infty}^{i}=0 \text { for } i>n^{\prime \prime} \text { and for } i \in\left\{i: n^{\prime}<i \leq n^{\prime \prime}, x^{* i}(\infty)>\bar{x}^{i}\right\}
\end{gathered}
$$

Finally, $\hat{p}_{\infty}=\sum_{j \in G(\infty)} \gamma^{j} \hat{g}_{x}^{j}(\infty)$ for some $\gamma^{j} \geq 0$ and if (6) fails, then (18) must be replaced by $\left(p_{0}, p_{\infty}, \sum_{j} \int_{(0, \infty)} \mathrm{d} v^{j}(t), \sum_{j \in G(\infty)} \gamma^{j}\right) \neq 0$.

Remark 1. For $i=j \in\left(n^{1}+1, \cdots, n\right)$, the growth condition in (13) can be weakened to : For some $\kappa_{*}<0$, for all $(t, x), \quad \kappa_{*} \leq f_{x^{i}}^{i}\left(t, x, u^{*}(t)\right) \leq \kappa \quad$ (where still $\kappa$ satisfies $\left.b>\left(n-n^{1}\right) \kappa\right)$.

In the sequel three trivial examples with rather obvious optimal controls will be presented, but to illustrate the use of the necessary conditions, we derive the form of the optimal controls from these conditions.

Example 1. $\max \int_{0}^{\infty} x \mathrm{e}^{-4 t} \mathrm{~d} t, \dot{x}=u x, \quad x(0)=1, \quad x(\infty)$ free, $u \in[1,2], \quad \dot{y}=x \mathrm{e}^{-3 t}, \quad y(0)=0, \quad y \leq 3 / 4$, $y(\infty)$ free.

\section{Solution:}

Evidently $x^{*}(t)>0$ and, because $\dot{y}>0$, by necessity, $y(t)<3 / 4$ for all $t$. For $p(t)=\left(p^{x}(t), p^{y}(t)\right)$, from (16) we get $\dot{p}^{x}=-p_{0} \mathrm{e}^{-4 t}-u p^{x}-p^{y} \mathrm{e}^{-3 t}, \dot{p}^{y}=0$. Then $p^{y}(t)=p^{y}(\infty)=\hat{p}_{\infty}^{y}=-\gamma^{y}=: k \leq 0$. The maximum condition is that

$$
u^{*}(t) \text { maximizes } p_{0} x^{*}(t) \mathrm{e}^{-4 t}+p^{x}(t) u x^{*}(t)+p^{y}(t) x^{*}(t) \mathrm{e}^{-3 t}
$$

Consider first the case that we might have $p_{0}=1, \quad k<0 \quad(\Rightarrow y(\infty)=3 / 4)$. Let $T_{*}$ satisfy $p_{0} \mathrm{e}^{-4 T_{*}}+k \mathrm{e}^{-3 T_{*}}=0$. Now, for $T>T_{*}, T>0, \quad \hat{p}_{\infty}^{x}=p_{\infty}^{x}=0$ means that $\dot{p}^{x}(t, T)>0$ for $t$ close to $T, t<T$, ${ }^{3} p(t, T)$ always exists, and is left continuous on $(0, T]$. 
see the expression for $\dot{p}^{x}$, so $p^{x}(t, T)<0$ for $t$ close to $T$. But this surely continues back to $\max \left\{0, T_{*}\right\}$ (see the expression for $\dot{p}^{x}$ again). So even $p^{x}(t)<0$ for $t \geq \max \left\{0, T_{*}\right\}$ (for such $t$, in fact, $p^{x}(t, T) \downarrow p^{x}(t)$ when $T \rightarrow \infty)$. Let $t^{*}$ be the smallest $t_{*}$ such that $p^{x}(t)<0$ for $t>t_{*}$. Consider first the subcase $t^{*}>0$. Then $p^{x}\left(t^{*}\right)=0$ and by the expression for $\dot{p}^{x}, \dot{p}^{x}\left(t^{*}\right)<0$ and $p^{x}(t)>0, t<t^{*}$. By the maximum condition $u=2$ for $t<t^{*}, u=1$ for $t>t^{*}$. We must have $t^{*}=\ln 2$, in order to obtain $y(\infty)=3 / 4$ : we get $x^{*}(t)=\mathrm{e}^{2 t}, t \leq t^{*}, \quad x^{*}\left(t^{*}\right)=\mathrm{e}^{2 t^{*}}=4, \quad x^{*}(t)=2 \mathrm{e}^{t}$ for $t>t^{*}, \quad y^{*}(t)=\int_{0}^{t^{*}} \mathrm{e}^{-t} \mathrm{~d} t+\int_{t^{*}}^{t} 2 \mathrm{e}^{-2 t} \mathrm{~d} t=1-1 / 2+1 / 4-\mathrm{e}^{-2 t}$ for $t>t^{*}$, so $y^{*}(\infty)=3 / 4$. For $T>t>t^{*}, \quad p^{x}(t, T)=p_{0}(1 / 3) \mathrm{e}^{-4 t}+C \mathrm{e}^{-t}+(k / 2) \mathrm{e}^{-3 t} \quad(C$ an arbitrary constant). Using $p^{x}(T-, T)=p_{\infty}^{x}+\hat{p}_{\infty}^{x}=0$, we get $C=C(T)=-p_{0}(1 / 3) \mathrm{e}^{-3 T}-(k / 2) \mathrm{e}^{-2 T}$, hence $C(\infty)=0$. Thus, $p^{x}(t)=\lim _{T} p^{x}(t, T)=p_{0}(1 / 3) \mathrm{e}^{-4 t}+(k / 2) \mathrm{e}^{-3 t}, t \geq t^{*}$, with $k$ satisfying $p^{x}\left(t^{*}\right)=0$, i.e. $(1 / 3)(1 / 16)+(k / 2)(1 / 8)=0$, so $k=-1 / 3$. (By the way, note here that as $p^{x}(t)$ satisfies the equation for $\dot{p}^{x}$, we would know that $p^{x}(t)=p_{0}(1 / 3) \mathrm{e}^{-4 t}+C \mathrm{e}^{-t}+(k / 2) \mathrm{e}^{-3 t}$ for some constant $C$, but $p^{x}(\infty)=p_{\infty}^{x}+\hat{p}_{\infty}^{x}=0$ would not determine the constant. This shows the usefulness of the formula $\left.p(t)=\lim _{T} p(t, T)\right)$ The subcase $t^{*}=0$ is impossible, then $u \equiv 1$, and $y^{*}(\infty)<3 / 4$. The case $p_{0}=1$, $k=0$ is impossible, then $\dot{p}^{x}(t, T)<0$ for $t$ close to $T$, so $p^{x}(t, T)>0$ for such $t$ and then for all $t$ (see the expression for $\left.\dot{p}^{x}\right)$. In fact, $p^{x}(t, T) \uparrow p^{x}(t)$ when $T \rightarrow \infty$, so $p^{x}(t)>0$ for all $t$, implying $u \equiv 2$, and $y^{*}(\infty)>3 / 4$, a contradiction. Consider finally $p_{0}=0$. Then, by (18), $\hat{p}_{\infty} \neq 0$, so $k<0$, and then $\dot{p}^{x}(t, T)>0$ for $t$ close to $T$, so $p^{x}(t, T)<0$ for such $t$, in fact for all $t$ (see the expression for $\dot{p}^{x}$ ), and $p^{x}(t, T) \downarrow p^{x}(t)$. Hence, $p^{x}(t)<0$ for all $t$, which gives $u \equiv 1$ and $y^{*}(\infty)<3 / 4$, contradicting $k<0$.

\section{Remark 2. (Further non-triviality properties)}

a) Replace (6) by the assumption that either $G(\infty)$ is empty, or (if not), for some $T^{\prime \prime}>0$, some $\varepsilon>0$, for any $s \geq T^{\prime \prime}$, there exists a $v_{s} \in \operatorname{cof}\left(s, x^{*}(s), U\right)$ such that $\hat{g}_{t}^{j}(\infty)+\hat{g}_{x}^{j}(\infty) v_{s} \geq \varepsilon$ for all $j \in G(\infty)$, where $\hat{g}_{t}^{j}(\infty):=\lim _{t \rightarrow \infty} g_{t}^{j}\left(t, x^{*}(t)\right)$ is assumed to exist. Assume also that $s \rightarrow v_{s}$ and $f^{i}\left(s, x^{*}(s), u^{*}(s)\right)$, $i=1, \cdots, n$, are bounded ${ }^{4}$. Then $\left(p_{0}, p_{\infty}, \sum_{j} \int_{(0, \infty)} \mathrm{d} v^{j}(t)\right) \neq 0$.

b) Assume in addition that, for any $s>0$, either $G(s)$ is empty, or (if not), there exists a $v_{s} \in \operatorname{cof}\left(s, x^{*}(s), U\right)$ such that $\hat{g}_{t}^{j}(s)+\hat{g}_{x}^{j}(s) v_{s}>0$ for all $j \in G(s)$ and, in case $j^{*}>1$, that for each $u$, $t \rightarrow f\left(t, x^{*}(t), u\right)$ is continuous, that $f^{i}\left(t, x^{*}(t), u^{*}(t)\right), \quad i=1, \cdots, n^{1}$, is left continuous at each $t>0$ and has a limit when $t \rightarrow \infty$. Then $\left(p_{0}, p_{\infty}\right) \neq 0$.

For finite horizon normality conditions, see [23] and [24].

The main reason for including the next theorem is that it forms a basis for obtaining Theorem 1, but it has some interest of its own.

It contains necessary conditions for the case where (14) and/or (15) fail, in particular where $g^{j}$ also depends on $x^{i}, \quad i>n^{1}$. We then need three conditions, see (25)-(27) below, that automatically hold if (14) and (15) are satisfied.

Theorem 2. In the situation of Theorem 1, with (5), (6), (8), (14), and (15) deleted, assume that the three conditions (25), (26), (27) below are satisfied. Then the following necessary conditions hold: for some $p_{0} \in\{0,1\}$, for some vector $p_{\infty}$ and some bounded nonnegative finitely additive set functions $\mu_{1}, \cdots, \mu_{j^{*}}$, vanishing on

${ }^{4}$ For $\quad i>n^{1} \quad$ it suffices that $\mathrm{e}^{-a s} f^{i}\left(s, x^{*}(s), u^{*}(s)\right) \rightarrow 0$ when $s \rightarrow \infty, \omega=(1 / 2)\left(b-\left(n-n^{1}\right) \kappa\right)$, note that for $i>n^{1}$, $p^{i}(s) \leq C \mathrm{e}^{-\omega s}$ for some $C$. 
sets of Lebesgue measure zero, for a.e. s, for all $u \in U$,

$$
p_{0}\left[v^{0}(s)+\int_{s}^{\infty} f_{x}^{0 *}(t) C(t, s) v(s) \mathrm{d} t\right]+p_{\infty} C(\infty, s) v(s)+\sum_{j} \int_{[s, \infty)} \hat{g}_{x}^{j}(t) C(t, s) v(s) \mathrm{d} \mu_{j}(t) \leq 0
$$

where $v(s):=f\left(s, x^{*}(s), u\right)-f^{*}(s), \quad v^{0}(s)=f^{0}\left(s, x^{*}(s), u\right)-f^{0 *}(s), \quad \hat{g}_{x}^{j}(t):=g_{x}^{j}\left(t, x^{*}(t)\right)(C(\infty, s)$ and the integrals exist). Moreover, $\left(p_{0}, p_{\infty}, \mu_{1}([0, \infty)), \cdots, \mu_{j^{*}}([0, \infty))\right) \neq 0, \quad p_{\infty}$ satisfying (20). Finally, defining $J(j, \beta):=\left\{t \in\left[a_{j}, b_{j}\right]: g^{j}\left(t, x^{*}(t)\right) \geq \beta\right\}$, we have

$$
\mu_{j}\left(\complement\left[a_{j}, b_{j}\right]\right)=0 \text { and } \mu_{j}(J(j, \beta))=0 \text { for all } \beta>0
$$

For $j \notin G(0+)$, for positive $s$ close enough to $0, \mu_{j}([0, s])=0$

If (7) and (8) hold, $\left(p_{0}, p_{\infty}, \lim _{s \rightarrow 0} \sum_{j} \mu_{j}([s, \infty))\right) \neq 0$. Moreover,

$\left(p_{0}, \liminf _{s \rightarrow \infty}|p(s)|, \lim _{s \rightarrow \infty} \sum_{j} \mu_{j}([0, s])\right) \neq 0$, if, for some $c^{*} \in C^{\infty}$ and some positive $\hat{T}, \delta$,

$g_{x}^{j}\left(t, x^{*}(t)\right) C(t, s) c^{*} \geq \delta$ when $t \geq s \geq \hat{T}$ for all $j \in G_{\infty} \backslash G_{*}^{\infty}, \quad G_{*}^{\infty}:=\left\{j \in G_{\infty}: \liminf _{t \rightarrow \infty} g^{j}\left(t, x^{*}(t)\right)>0\right\}$.

Finally, if both the last condition and (7) and (8) hold, then

$$
\left(p_{0}, \liminf _{s \rightarrow \infty}|p(s)|, \lim _{s \rightarrow \infty} \sum_{j} \mu_{j}([1 / s, s])\right) \neq 0 \text {. }
$$

As before when $b_{j}=\infty,\left[a_{j}, b_{j}\right]$ is replaced by $\left[a_{j}, \infty\right)$.

Assume, for some arbitrarily large $T^{\prime}>0$, that the conditions (25)-(27) hold

$$
\text { For all } j, t \rightarrow g_{x}^{j}\left(t, x^{*}(t)\right) C\left(t, T^{\prime}\right) \text { is bounded on }\left[T^{\prime}, \infty\right)
$$

Let $x^{* *}(t)$ be a solution on $\left[T^{\prime}, \infty\right)$ of $\dot{x}=f\left(t, x, u^{*}(t)\right)$ for $x^{* *}\left(T^{\prime}\right)$ given. For some positive second order term $o_{1}(z)$ (i.e. $\lim _{z \downarrow 0} o_{1}(z) / z=0$ ), if $\left|x^{* *}\left(T^{\prime}\right)-x^{*}\left(T^{\prime}\right)\right| \leq d$, then, for all $j$, for all $t \geq T^{\prime}$

$$
g^{j}\left(t, x^{* *}(t)\right)-g^{j}\left(t, x^{*}(t)\right)-g_{x}^{j}\left(t, x^{*}(t)\right)\left(x^{* *}(t)-x^{*}(t)\right) \geq-o_{1}(d)
$$

Moreover, for any given number $K^{\prime}$ and any given positive second order term $o_{2}(d)$, a positive second order term $o_{3}(d)$ exists such that the following property holds. Let $q(t)$ be a solution on $\left[T^{\prime}, \infty\right)$ of $\dot{q}=f_{x}^{*}(t) q$, for $q\left(T^{\prime}\right)$ given, $\left|q\left(T^{\prime}\right)\right| \leq K^{\prime}$. Then, if $\left|x^{* *}\left(T^{\prime}\right)-x^{*}\left(T^{\prime}\right)-d q\left(T^{\prime}\right)\right| \leq o_{2}(d)$

$$
\text { for any } t \geq T^{\prime}, g_{x}^{j}\left(t, x^{*}(t)\right)\left(x^{* *}(t)-x^{*}(t)-d q(t)\right) \geq-o_{3}(d)
$$

As an example in which (25)-(27) hold, consider a case where $n^{1}=0$, (11) and (12) hold for $i=0$, where $f$ is concave in $x$, and where, for some positive $\kappa, \kappa^{\prime}, \kappa^{*}, g^{j}=\mathrm{e}^{-n \kappa^{\prime}} h^{j}(x), h^{j}$ is $C^{1}$ and convex, $0 \leq-h_{x^{i}}^{j}(x) \leq \kappa^{*}$, and $0 \leq f_{x^{j}}^{i}(t, x, u) \leq \kappa \leq \kappa^{\prime}, \quad i>0$, all $j, b>n \kappa$. (For $z=x^{* *}-x^{*}-d q(t)$, in a short hand notation

$$
\begin{aligned}
\dot{z}^{i} & =f^{i}\left(z+x^{*}+d q\right)-f^{i}\left(x^{*}\right)-f_{x}^{i}\left(x^{*}\right) d q \\
& \leq f^{i}\left(z+x^{*}+d q\right)-f^{i}\left(x^{*}+d q\right)+f^{i}\left(x^{*}+d q\right)-f^{i}\left(x^{*}\right)-f_{x}^{i}\left(x^{*}\right) d q \\
& \leq \sum_{j} f_{x^{j}}^{i}\left(x^{*}+d q\right) z^{j} \leq n \kappa z_{*}
\end{aligned}
$$

where $z_{*}:=\max \left\{0, z^{1}, \cdots, z^{n}\right\}$, which means that, a.e., $\dot{z}_{*} \leq n \kappa z_{*}(t)$, so $z_{*}(t) \leq z_{*}\left(T^{\prime}\right) \mathrm{e}^{n \kappa\left(t-T^{\prime}\right)}, \quad t>T^{\prime}$. If $\left|z\left(T^{\prime}\right)\right| \leq o_{2}(d)$, then $0 \leq z_{*}\left(T^{\prime}\right) \leq \max _{i}\left|z^{i}\left(T^{\prime}\right)\right| \leq o_{2}(d)$ and then $-g_{x}^{j} z(t) \leq \sum_{i}-g_{x^{i}}^{j} \max \left(0, z^{i}(t)\right) \leq \mathrm{e}^{-n \kappa^{\prime} t} j^{*} \kappa^{*} z_{*}\left(T^{\prime}\right) \mathrm{e}^{n \kappa t} \leq j^{*} \kappa^{*} O_{2}(d)$

Remark 3. For Theorem 2 to hold, we can weaken (7) and the basic assumptions on $f_{x}^{i}$ and $g_{x}^{j}$ as follows: the derivatives $g_{x}^{j}$ and $f_{x}^{i}$ exist at $\left(t, x^{*}(t)\right)$ for all $t$ and the three conditions on $g^{j}$ below are satisfied: 
For all $N>0$

$$
\begin{gathered}
x \rightarrow g^{j}(t, x) \text { is differentiable at }\left(t, x^{*}(t)\right), \text { uniformly in } t \in[0, N] \\
\quad(t, x) \rightarrow g^{j}(t, x) \text { is differentiable at }\left(t, x^{*}(t)\right) \text { for all } t \\
t \rightarrow\left(g^{j}\left(t, x^{*}(t)\right), g_{x}^{j}\left(t, x^{*}(t)\right), g_{t}\left(t, x^{*}(t)\right)\right) \text { is continuous }
\end{gathered}
$$

Moreover in the growth conditions (12) and (13), roughly speaking, the inequalities need not hold for states $x$ that cannot possibly occur, more precisely, the conditions can be modified as follows. Define for each $t$,

$X_{\delta}(t):=\operatorname{co}\left\{x^{u(.)}(t): u(.) \in \mathcal{U}, \sigma\left(u(),. u^{*}().\right) \leq \delta, x^{u}(\right.$.$\left.) exists on [0, t]\right\}, \quad \sigma\left(u(),. u^{*}().\right):=$ meas $\left\{t: u(t) \neq u^{*}(t)\right\}$, $x^{u(.)}($.$) the solution of (2) corresponding to u($.$) . Then (12), (13) need only hold for some \delta>0$, for $(t, x)$ such that $x \in X^{\delta}(t):=B\left(X_{\delta}(t), \delta\right):=\left\{x: \operatorname{dist}\left(x, X_{\delta}(t)\right)<\delta\right\}$. In $(10)(\gamma)$ we must add the assumptions that, for some $\theta>0, g^{j}(t, x)$ is differentiable in $(t, x)$ at $\left(t, x^{*}(t)\right)$ uniformly in $t \in[0, \theta]$, with a derivative at this point bounded uniformly in $t \in[0, \theta]$.

Finally, $U$ can be replaced by a time dependent subset $\tilde{U}(t):=\left\{u^{*}(t)\right\} \cup \breve{U}(t)$, where $\breve{U}(t):=\left\{u \in U: \phi_{k}(u, t)>0\right.$ for all $k$ such that $\left.t \in\left[a^{k}, b^{k}\right]\right\}, \quad \phi_{k}, k=1, \cdots, k^{\prime}$, continuous, we then require all control functions to satisfy $u(t) \in \tilde{U}(t)$. We assume $\breve{U}(0) \neq \varnothing$, and in case $G(0+) \neq \varnothing$, in (9), we require $v^{*} \in \operatorname{cof}\left(0, x_{0}, \breve{U}(0)\right)$. Then the maximum conditions (17) and (22) hold only for $u \in \breve{U}(t)$. (The set $U$ can even be replaced by $\left\{u^{*}(t)\right\} \cup \mathrm{cl} \breve{U}(t)$, with (17) and (22) holding for $u \in \mathrm{cl} \breve{U}(t)$. We must then still require $\breve{U}(0) \neq \varnothing$, and, in (9), $v^{*} \in \operatorname{cof}\left(0, x_{0}, \breve{U}(0)\right)$. In the proof below, the perturbations $u_{i}$ of the optimal control have to belong to $\breve{U}(t)$.

\section{Example 2.}

$\max \int_{0}^{\infty} u \mathrm{e}^{-2 t} \mathrm{~d} t, \quad u \in[-2,2], \quad x(t) \geq 0$ for all $t, \dot{x}=x-u, \quad x(0)=1$, $x(\infty)$ free. It is convenient to replace $x \geq 0$ by $g=\mathrm{e}^{-t} x \geq 0$ (Then (25)-(27) will be satisfied). Choose $t^{*}$ as the largest possible $t_{*}$ such that $x^{*}(t)>0$ for $t<t_{*}$. If $t^{*}=\infty$, and even, for some $\varepsilon>0$, $\mathrm{e}^{-t} x^{*}(t) \geq \varepsilon$ for all $t$, then $p_{0}=1, \quad 0=p(t, T)=p(t)$, so $u \equiv 2$ by the maximum condition

$$
u^{*}(s) \text { maximizes } p_{0} u \mathrm{e}^{-2 s}+p(s)\left(x^{*}(s)-u\right)
$$

and $x^{*}(t)=2-\mathrm{e}^{t}$, contradicting the last inequality. Consider now the case where $\gamma:=\inf _{t \in[0, \infty)} \mathrm{e}^{-t} x^{*}(t)=0$. Let $\tilde{J}$ be the set of time points $s$ for which (31) holds. Now, $\gamma=0$ implies the existence of some $t^{\prime}=s \in \tilde{J} \cap\left[0, t^{*}\right)$ for which $u^{*}\left(t^{\prime}\right)>-2^{5},\left(x^{*}(t) \mathrm{e}^{-t}=x_{0}-\int_{0}^{t} u^{*}(r) \mathrm{e}^{-r} \mathrm{~d} r\right.$, see the state equation). If $t^{*}=\infty$, $t^{\prime}$ can be chosen arbitrarily large, if $t^{*}<\infty, t^{\prime}$ can be chosen arbitrarily close to $t^{*}$. Now, $C(s, t)=\mathrm{e}^{s}-t$, so $\hat{g}_{x}(s) C(s, t)=\mathrm{e}^{-t}$. If $p_{0}=0$, then $\int_{t^{\prime}}^{\infty} \mathrm{e}^{-s} C\left(s, t^{\prime}\right)\left\{-\left[-2-u^{*}\left(t^{\prime}\right)\right]\right\} \mathrm{d} \mu(s)=\mathrm{e}^{-t^{\prime}} \mu\left(\left[t^{\prime}, \infty\right)\right)\left[2+u^{*}\left(t^{\prime}\right)\right] \leq 0$ (use (31) for $u=-2)$, implying $\mu\left(\left[t^{\prime}, \infty\right)\right)=0$, contradicting $\left(p_{0}, \mu\right) \neq 0$. Hence, $p_{0}=1$ (Note that this argument would not work if we had replaced $x \geq 0$, by, say, $\mathrm{e}^{-2 t} x \geq 0$ ). For $u=-2$, by (31), $\mathrm{e}^{-2 t^{\prime}}-\mathrm{e}^{-t^{\prime}} \mu\left(\left[t^{\prime}, \infty\right)\right) \geq 0$ and because $\mathrm{e}^{-2 t}-\mathrm{e}^{-t} \mu([t, \infty))$ is strictly decreasing on $\left[0, t^{*}\right)(\mu([t, \infty))$ is constant on $\left[0, t^{*}\right)$ ), in fact $u^{*}=2$ on $\left[0, t^{\prime}\right)$, in fact on all $\left[0, t^{*}\right)$, by (31). Hence, on $\left[0, t^{*}\right)$,

${ }^{5}$ Or even $u^{*}\left(t^{\prime}\right)>0$, an observation needed in the next example. 
$x^{*}(t)=-\mathrm{e}^{t}+2$, so $t^{*}$ is finite and $x^{*}\left(t^{*}\right)=0$ and $t^{*}=\ln 2$. It is easily seen by a similar argument that on $\left(t^{*}, \infty\right), \quad u^{*}=0$ : to see this, having $\mathrm{e}^{-t} x^{*}(t)>0$ on an interval $(a, b)$, assuming that $(a, b)$ is as large as possible, is impossible: Let $c \in(a, b)$, and define $\gamma_{c}:=\inf _{t} \in[c, \infty) \mathrm{e}^{-t} x^{*}(t)$. Now, $\gamma_{c}>0$ leads to $p(t)=0, \quad u^{*}=2$ on $[c, \infty)$, which is impossible, and both for $b<\infty$ and $x^{*}(b)=0$, and for $b=\infty$ and $\gamma_{c}=0$, certain time points in $(a, b) \cap \tilde{J}$ close to $b$ (i.e. arbitrarily large if $b=\infty$ ) exist at which $u^{*}>-2$, but then $u^{*}=2$ in $(a, b)$ by $(31)$, as $\mathrm{e}^{-2 t}-\mathrm{e}^{-t} \mu([t, \infty))$ is strictly decreasing in $(a, b)$. But $u^{*}=2$ in $(a, b)$ contradicts $x^{*}(a)=0, \quad x^{*}(t)>0$ in $(a, b)$. Hence, on $\left(t^{*}, \infty\right), \mathrm{e}^{-2 t}=\int_{[t, \infty)} \mathrm{e}^{s}-t \mathrm{e}^{-s} \mathrm{~d} \mu(s)$ by (31), so $\mathrm{e}^{-t}=\int_{[t, \infty)} \mathrm{d} \mu(s)$, or $\mathrm{e}^{-t}=\dot{\mu}$ on $\left(t^{*}, \infty\right)$ ( $\mu$ can be represented by an integrable function $\dot{\mu}$ here). If we put $\mu\left(\left\{t^{*}\right\}\right):=\inf _{n \geq 1} \mu\left(\left[t^{*}-1 / n, t^{*}+1 / n\right]\right)=0$, we have a valid proposal for the multipliers (It can be seen that $\mu\left(\left\{t^{*}\right\}\right)=0$ is even necessary, compare (89(ii)) on p. 333 in [6]).

\section{Example 3.}

$\max \int_{0}^{\infty} 2 u^{1 / 2} \mathrm{e}^{-2 t} \mathrm{~d} t, \quad u \in[0, \infty), \quad x \geq 0, \quad \dot{x}=x-u, \quad x(0)=1, \quad x(\infty)$ free. It is convenient to replace $x(t) \geq 0$ by $\mathrm{e}^{-t} x(t) \geq 0$. The maximum condition is

$$
u^{*}(s) \text { maximizes } p_{0} 2 u^{1 / 2} \mathrm{e}^{-2 s}+p(s)\left(x^{*}(s)-u\right)
$$

Again, assuming, for some $\varepsilon>0$ that $\mathrm{e}^{-t} x^{*}(t) \geq \varepsilon$ for all $t$, and (in the opposite case) $p_{0}=0$ both yield contradictions. So $p_{0}=1$. Now, $u^{*}=0$ in (32) is impossible, so $u^{*}>0$ all the time. But, due to the constraint $\mathrm{e}^{-t} x \geq 0$, then $x^{*}(t)>0$ all the time (see the state equation). Let $\mu(\infty)=\inf _{s \geq 0} \mu([s, \infty))$. The maximum condition (32) yields $\mathrm{e}^{-2 t} / u^{* 1 / 2}=\mu(\infty) \mathrm{e}^{-t}, \quad u^{*}=\left[\mathrm{e}^{-t} / \mu(\infty)\right]^{2}=a \mathrm{e}^{-2 t}$, for $a=1 / \mu(\infty)^{2}$, $\mu(\infty) \neq 0$. Now, $\frac{\mathrm{d} x^{*}}{\mathrm{~d} t}=x^{*}-a \mathrm{e}^{-2 t}$. The general solution is $x^{*}(t)=a \mathrm{e}^{-2 t} / 3+C \mathrm{e}^{t}$. To have the initial condition and $\liminf _{t \rightarrow \infty} \mathrm{e}^{-t} x^{*}(t)=0 \quad(\mu(\infty) \neq 0)$ satisfied we need $a=3, \quad C=0$, and hence $\mu(\infty)=(3)^{-1 / 2}$.

Remark 4. Assume in the problem (1)-(7), (11)-(15), that $U$ is convex and that there are given additional constraints in the problem of the form $g^{j}(t, x, u) \geq 0, \quad t \in\left[a_{j}, b_{j}\right], j=j^{*}+1, \cdots, j^{* *}$. (Perhaps $b_{j}=\infty$. In this case, here and below, replace $\left[a_{j}, b_{j}\right]$ by $\left[a_{j}, \infty\right)$.) Assume that $\left(x^{*}(),. u^{*}().\right)$ is optimal in this problem. We assume for $j>j^{*}$ that $g^{j}$ is continuous and depends only on $x^{i}, \quad i \leq n^{1}$, that $g_{x}^{j}$ and $g_{u}^{j}$ exist, that $\sup _{t}\left|g_{x}^{j}\left(t, x^{*}(t), u^{*}(t)\right)\right|<\infty, \sup _{t}\left|g_{u}^{j}\left(t, x^{*}(t), u^{*}(t)\right)\right|<\infty$, that $(z, v) \rightarrow\left(g_{x}^{j}\left(t, x^{*}(t)+z, u^{*}(t)+v\right), g_{u}^{j}\left(t, x^{*}(t)+z, u^{*}(t)+v\right)\right)$ is continuous in $(x, v)$, uniformly in $t$ and that $f^{i}$ is $C^{1}$ in $(x, u)$ and measurable in $t$. We assume, for some positive constants $\tilde{\delta}, A, a, B, b, C, \kappa$, for all $t, x, u \in B\left(u^{*}(t), \tilde{\delta}\right)$, that for $i=0, \cdots, n^{1}, \quad\left|f_{u}^{i}(t, x, u)\right| \leq A \mathrm{e}^{-a t}$ and $\left|f_{x^{j}}^{i}(t, x, u)\right| \leq A \mathrm{e}^{-a t}$ for $j \leq n^{1}$ and $\left|f_{x^{j}}^{i}(t, x, u)\right| \leq B \mathrm{e}^{-b t}$ for $j>n^{1}$, and, for $i>n^{1}$, that $\left|f_{u}^{i}(t, x, u)\right| \leq C \mathrm{e}^{\kappa t}$ and $\left|f_{x^{j}}^{i}(t, x, u)\right| \leq C \mathrm{e}^{\kappa t}$ for $j \leq n^{1}$ and $\left|f_{x^{j}}^{i}(t, x, u)\right| \leq \kappa$ for $j>n^{1}, \quad b>\kappa\left(n-n^{1}\right)$. Define $U(t):=\left\{u \in U:\right.$ For all $j>j^{*}$ such that $\left.t \in\left[a_{j}, b_{j}\right], \quad g^{j}\left(t, x^{*}(t), u\right)>0\right\}$. Assume that $U(0) \neq \varnothing$, and that (8) holds, for $j \leq j^{*}$, for $F(0):=f(t, x, U(0))$ in (9). Write $\hat{g}_{x}^{j}(t)=g_{x}^{j}\left(t, x^{*}(t), u^{*}(t)\right), \quad \hat{g}_{u}^{j}(t)=g_{u}^{j}\left(t, x^{*}(t), u^{*}(t)\right), j>j^{*}$. Then, in addition to $p_{0} \in\{0,1\}, \quad p_{\infty}$, and $\mu_{j} \geq 0$ satisfying (20), (23), and (24), $j \leq j^{*}$, there exist bounded nonnegative finitely additive set functions $\mu_{j}, \quad j>j^{*}$, also vanishing on Lebesgue null sets, such that (22) holds 
for $u \in U(t)$, summing now over $j=1, \cdots, j^{* *}$. Moreover, for $C^{0}(t, s)=\int_{s}^{t} f_{x}^{0 *}(r) C(r, s) \mathrm{d} r$, for all

$$
\begin{gathered}
w(.) \in W^{*}:=\left\{\hat{w}(.) \in L_{\infty}\left([0, \infty), \mathbb{R}^{\tilde{k}}\right): u^{*}(t)+\hat{w}(t) \in U \text { for all } t\right\} \\
\begin{array}{c}
0 \geq p_{0}\left[\int_{0}^{\infty} f_{u}^{0 *}(t) w(t) \mathrm{d} t+\int_{0}^{\infty} C^{0}(\infty, t) f_{u}^{*}(t) w(t) \mathrm{d} t\right]+p_{\infty} \int_{0}^{\infty} C(\infty, t) f_{u}^{*}(t) w(t) \mathrm{d} t \\
\quad+\sum_{j=1}^{j^{* *}} \int_{[0, \infty)}\left[\hat{g}_{x}^{j}(t) \int_{0}^{t} C(t, r) f_{u}^{*}(r) w(r) \mathrm{d} r+\hat{g}_{u}^{j}(t) w(t)\right] \mathrm{d} \mu_{j}(t)
\end{array}
\end{gathered}
$$

$\left(C^{0}(\infty, t), C(\infty, s)\right.$ and the integrals exist). Furthermore, for $j>j^{*}$ we have $\mu_{j}=0$ on $C\left[a_{j}, b_{j}\right]$ and on $J(j, \beta):=\left\{t \in\left[a_{j}, b_{j}\right]: g^{j}\left(t, x^{*}(t), u^{*}(t)\right) \geq \beta\right\}$ for all $\beta>0$. Finally,

$\left(p_{0}, \liminf _{s \rightarrow \infty}|p(s)|, \lim _{s \downarrow 0} \hat{\mu}([s, 1 / s)), \breve{\mu}\right) \neq 0$, where $\hat{\mu}=\left(\mu_{1}, \cdots, \mu_{j^{*}}\right), \breve{\mu}=\left(\mu_{j^{*}+1}, \cdots, \mu_{j^{* *}}\right)$. (If (6) fails, then the last property must be replaced by $\left.\left(p_{0}, p_{\infty}, \lim _{s \downarrow 0} \hat{\mu}([s, \infty)), \breve{\mu}\right) \neq 0\right)$.

When for some vectors $g_{\infty}^{j}, j>j^{*}, \quad \limsup _{t \rightarrow \infty}\left|\hat{g}_{x}^{j}(t)-g_{\infty}^{j}\right|=0$, the following properties hold: the maximum condition (17) holds for $u \in U(t)$, together with

$$
\int_{0}^{\infty} H_{u}\left(t, x^{*}(t), u^{*}(t), p_{0}, p(t)\right) w(t) \mathrm{d} t+\sum_{j>j^{*}} \int_{[0, \infty)} \hat{g}_{u}^{j}(t) w(t) \mathrm{d} \mu_{j}(t) \leq 0
$$

for all $w(.) \in W^{*}$, where now $p(s)=\lim _{T \rightarrow \infty} p(s, T)$

$$
p(s, T)=p_{\infty}+\hat{p}_{\infty}+\breve{p}_{\infty}+\int_{s}^{T} H_{x}\left(t, x^{*}(t), u^{*}(t), p_{0}, p(t, T)\right) \mathrm{d} t+\sum_{j=1}^{j^{* *}} \int_{[s, T} \hat{g}_{x}^{j}(t) \mathrm{d} \mu_{j}(t)
$$

and where, for some nonnegative $\gamma^{j}, j=1, \cdots, j^{* *}, \quad \hat{p}_{\infty}=\sum_{j \leq j^{*}} \gamma^{j} \hat{g}_{x}^{j}(\infty), \quad \breve{p}_{\infty}=\sum_{j>j^{*}} \gamma^{j} g_{\infty}^{j}, \quad \gamma^{j}=0$ for $j \notin G(\infty), j \leq j^{*}, \quad$ and $\quad \gamma^{j}=0 \quad$ for $\quad j>j^{*} \quad$ if $\quad b_{j}<\infty \quad$ or $\quad$ if $\quad \liminf _{t \rightarrow \infty} g^{j}\left(t, x^{*}(t), u^{*}(t)\right)>0$ for $j \in\left\{j>j^{*}: b_{j}=\infty\right\}$. Moreover, $\inf _{s \geq 0} \mu_{j}([s, \infty))=: \mu_{j}(\infty)=\gamma^{j}$, and in (36), for $j \leq j^{*}, \mu_{j}$ can be represented by a bounded nondecreasing right-continuous function $v^{j}$, and, finally, $\left(p_{0}, p_{\infty}+\hat{p}_{\infty}, \sum_{j \leq j^{*}} \int_{(0, \infty)} \mathrm{d} v^{j}(t), \breve{\mu}\right) \neq 0$. (If (6) fails, then this property must be replaced by $\left.\left(p_{0}, p_{\infty}, \sum_{j} \int_{(0, \infty)} \mathrm{d} v^{j}(t), \sum_{j \in G(\infty), j \leq j^{*}} \gamma^{j}, \breve{\mu}\right) \neq 0\right)$.

When, in addition, for some $\tilde{w}(.) \in W^{*}$, some $\delta>0, \quad \beta>0$, for all $j>j^{*}$, the inequality $\left(\hat{g}_{u}^{j}(t) \tilde{w}(t)-\delta\right) 1_{\left[a_{j}, b_{j}\right] \cap C J(j, \beta)} \geq 0$ holds for all $t$, then $\mu_{j}(\infty)=0$ for $j>j^{*}$, and, for $j>j^{*}$, in both (36) and (35), $\mu_{j}$ can be represented by a nonnegative function $\mu^{j}($.$) in L_{\infty}([0, \infty), \mathbb{R}) \cap L_{1}([0, \infty), \mathbb{R})$ (replace $\mathrm{d} \mu_{j}(t)$ by $\left.\mu^{j}(t) \mathrm{d} t\right)$ and, moreover, $\left(p_{0}, p_{\infty}+\hat{p}_{\infty}, \sum_{j \leq j^{*}} \int_{(0, \infty)} \mathrm{d} v^{j}(t)\right) \neq 0$ (If (6) fails, then this property must be replaced by $\left.\left(p_{0}, p_{\infty}, \sum_{j} \int_{(0, \infty)} \mathrm{d} v^{j}(t), \sum_{j \in G(\infty), j \leq j^{*}} \gamma^{j}\right) \neq 0\right)$. Finally, in this case, for a.e. $t$, for all $u \in U$

$$
H_{u}\left(t, x^{*}(t), u^{*}(t), p(t)\right)\left(u-u^{*}(t)\right)+\sum_{j>j^{*}} \hat{g}_{u}^{j}(t) \mu^{j}(t)\left(u-u^{*}(t)\right) \leq 0
$$

\section{Proofs of the Results}

Proof of Theorem 2. To simplify the notation, instead of the criterion (1), we can and shall assume that $a x(\infty)$ is the criterion to be maximized, $a=(1,0, \cdots, 0) \in \mathbb{R}^{n}$, that $x^{1}(\infty)$ is free, hence is not required to be equal to $\bar{x}^{1}$. The proof will be carried out under the assumptions of Theorem 2, allowing for the weakening of these as- 
sumptions in Remark 3.

Overview of the proof. A rough outline of the proof is as follows. We are going to make a number of strong (needleshaped) perturbations of $u^{*}($.$) . This gives rise to first order variations of the optimal trajectory (the q^{*}-$ functions below). We introduce a convex subset of these variations ( $Q_{\tau}^{\prime}$ below) consisting of variations satisfying a first order version of the unilateral constraint. We then introduce the convex set of endpoints (at infinity) of these variations as is standard in traditional proofs of the maximum principle, and show that it has to be separated from the set of "better, first order admissible" points, the set $\left\{\left(\delta_{1}, \cdots, \delta_{n^{\prime \prime}}\right): \delta_{1}>0, \delta_{i}=0\right.$ for $i \leq n^{\prime}, \delta_{i} \geq 0$ if $\left.x^{*^{i}}(\infty)=\bar{x}^{i}, n^{\prime \prime} \geq i>n^{\prime}\right\}$ (The endpoints we consider consist actually only of the first $n^{\prime \prime}$ components of the state). The separation argument (carried out in $\mathbb{R}^{n^{\prime \prime}}$ ) consists of a standard use of the Brouwer fixed point theorem combined with the fact the endpoints are "good" first order approximations of the endpoints of the exact solutions following from the perturbations. We need the fact that these exact solutions satisfy the unilateral state constraint, and this is shown first. The separating functional ( $n^{\prime \prime}$-vector) is denoted $p_{\infty}$. Another separation argument, carried out in $L_{\infty}$-space' gives the multipliers $\mu_{j}$ related to the unilateral state constraints.

Detailed proof. To avoid certain problems connected with coinciding perturbation time points, the following construction is helpful (we then avoid coinciding perturbation time points). Let $U^{\prime}$ be a countable dense set in $U$ and let $J^{\prime}$ be the set of right Lebesgue points of $f^{*}($.$) and all f\left(t, x^{*}(t), u\right), u \in U^{\prime}$ in $[0, \infty) \backslash\left\{b_{1}, \cdots, b_{j^{*}}\right\}$. Then choose some set $J^{\prime \prime} \subset J^{\prime} \backslash\left\{0, a_{1}, \cdots, a_{j^{*}}\right\}$ of full measure (i.e. meas $\left.\left([0, \infty) \backslash J^{\prime \prime}\right)=0\right)$, such that for each $u \in U^{\prime}$, a subset $J_{u}$ of $J^{\prime \prime}$ exists with the property that if $u, u^{\prime} \in U^{\prime}$ and $u \neq u^{\prime}$, then $J_{u} \cap J_{u^{\prime}}=\varnothing$, and with the property that for each $s \in J^{\prime \prime}$, each $u \in U^{\prime}$, there exists a sequence $s_{n} \rightarrow s$, such that $f^{*}\left(s_{n}\right) \rightarrow f^{*}(s)$ and $f\left(s_{n}, x^{*}\left(s_{n}\right), u\right) \rightarrow f\left(s, x^{*}(s), u\right), s_{n} \in J_{u}$. For any given $\tau \in J^{\prime}$,

$\tau \in\left\{t: \hat{g}^{j}(s)>0\right.$ for $\left.s \in(0, t], t \in J^{j}, j \notin G(0+)\right\}$, let $D_{m}^{\tau}$ be the collection of $m$-tuples of the type $P=\left(\left(s_{1}, u_{1}\right), \cdots,\left(s_{m}, u_{m}\right)\right), \quad \tau=s_{1} \leq s_{2} \leq \cdots \leq s_{m}, \quad u_{i} \in U, \quad s_{i} \in J^{\prime}$, and for all $i$ such that $s_{i}>\tau$, $u_{i}$ belongs to $U^{\prime}$ and $s_{i}$ belongs to $J_{u_{i}}$ (This means that for any $\left(s_{i^{\prime}}, u_{i^{\prime}}\right), \quad\left(s_{i}, u_{i}\right), \quad$ if $s_{i^{\prime}}=s_{i}>\tau$, then $J_{u_{i^{\prime}}} \cap J_{u_{i}} \neq \varnothing$, which implies $\left.u_{i^{\prime}}=u_{i}\right)$. The separate treatment of the case $s_{i}=\tau$, where we can have several perturbations at the same time, is useful for obtaining nondegeneracy results (i.e. informative necessary conditions). Below $m$ is varying $(m \geq 1)$.

Let $E_{m}:=\left\{c=\left(c_{1}, \cdots, c_{m}\right): c_{i}>0, \sum c_{i}<1\right\} \quad$ and for $c=\left(c_{1}, \cdots, c_{m}\right) \in E_{m}, \quad P=\left(\left(s_{1}, u_{1}\right), \cdots,\left(s_{m}, u_{m}\right)\right) \in D_{m}^{\tau}$, define

$$
\hat{c}_{i}=c_{i} / \breve{C}_{i}, \breve{C}_{i}=\sum_{i^{\prime} \in\left\{i^{\prime}: s^{i^{\prime}}=s^{i}\right\}} C_{i^{\prime}}
$$

and

$$
\begin{gathered}
q_{*}(t, c, P)=\sum_{i \in\left\{i: s_{i}+\breve{c}_{i} \leq t\right\}} C\left(t, s_{i}\right) c_{i}\left(f\left(s_{i}, x^{*}\left(s_{i}\right), u_{i}\right)-f^{*}\left(s_{i}\right)\right) \\
q^{*}(t, c, P)=\sum_{i \in\left\{i: s_{i} \leq t\right\}} C\left(t, s_{i}\right) c_{i}\left(f\left(s_{i}, x^{*}\left(s_{i}\right), u_{i}\right)-f^{*}\left(s_{i}\right)\right) \\
q^{* *}(t, c, P)=\sum_{i \in\left\{i: s_{i}+\breve{c}_{i} \leq t\right\}} C\left(t, s_{i}\right) c_{i}\left(f\left(s_{i}, x^{*}\left(s_{i}\right), u_{i}\right)-f^{*}\left(s_{i}\right)\right) \\
+\sum_{i} C\left(t, s_{i}\right) \hat{c}_{i}\left(t-s_{i}\right)\left(f\left(s_{i}, x^{*}\left(s_{i}\right), u_{i}\right)-f^{*}\left(s_{i}\right)\right) 1_{\left[s_{i}, s_{i}+\breve{c}_{i}\right)}(t)
\end{gathered}
$$

where a sum over an empty set is put equal to zero. For $t \notin \bigcup_{i}\left[s_{i}, s_{i}+\breve{c}_{i}\right), q^{*}(t, c, P)=q_{*}(t, c, P)=q^{* *}(t, c, P)$. Let $Q_{\tau}^{\prime \prime}:=\left\{q^{*}(., c, P): c \in E_{m}, P \in D_{m}^{\tau}, m \in\{1,2, \cdots\}\right\}$ and note that $Q_{\tau}^{\prime \prime}$ is convex 6 . Define $Q_{\tau}^{\prime}$ to be the convex subset of $Q_{\tau}^{\prime \prime}$ consisting of functions $q^{*}(., c, P)$ that satisfy: For some $\alpha>0, \beta>0$

$$
\text { for all } j, \hat{g}_{x}^{j}(t) q^{*}(t, c, P) \geq \alpha \text { for all } t \in J^{*}(j, \beta), t \geq \tau
$$

where $J^{*}(j, \beta):=\left\{t: g^{j}\left(t, x^{*}(t)\right) \leq \beta, t \in J^{j}\right\}$.

The linear variations $q^{*}(., c, P)$ are the ones that will appear in the necessary conditions that will be ob-

\footnotetext{
${ }^{6}$ One may consult Observation 1 below at this point.
} 
tained (see (53), (54) below). These variations are jumping at each perturbation time points, so, near these points, they do not approximate (to the first order) the corresponding (continuous) exact solutions. Yet, we are able to show that the latter solutions satisfy the unilateral constraints when $q^{*}(., c . P)$ belongs to $Q_{\tau}^{\prime}$. To show this, the "better", continuous, approximations $q^{* *}(., c, P)$ are used.

\subsection{Satisfaction of the Unilateral Constraints by Perturbed Solutions}

Fix a pair $\left(c^{*}, P^{*}\right)$ such that $q^{*}\left(., c^{*}, P^{*}\right)$ satisfies (38) for certain numbers $\alpha, \beta$, $P^{*}=\left(\left(s_{1}^{*}, u_{1}^{*}\right), \cdots,\left(s_{m^{*}}^{*}, u_{m^{*}}^{*}\right)\right), \quad c^{*}=\left(c_{1}^{*}, \cdots, c_{m^{*}}{ }^{*}\right)$. Let $T^{\prime} \geq \max _{i} s_{i}^{*}+1$ be some number for which (25), (26) and (27) hold. There exists a $\gamma>0$ so small that

(38) holds for $\alpha$ replaced by $\alpha / 2$ when $c \in B\left(c^{*}, \gamma\right), P=P^{*}$

To see this, choose $\gamma$ such that (39) is satisfied in this manner both for $t \in\left(0, T^{\prime}\right]$ and (by using (25)) for $t>T^{\prime}$. Then, for $d \in(0,1], \quad c \in B\left(c^{*}, \gamma\right), \quad q^{*}\left(., d c, P^{*}\right)$ satisfies

$$
\text { for all } j, \hat{g}_{x}^{j}(t) q^{*}(t, d c, P) \geq d \alpha / 2 \text { for all } t \in J^{*}(j, \beta), t \geq \tau
$$

For some positive $d_{1}$, for $d \in\left(0, d_{1}\right]$, for all $i, i^{\prime}$, when $s_{i} \neq s_{i^{\prime}}$, then $\left[s_{i}^{*}, s_{i}^{*}+d \breve{c}_{i^{\prime}}\right]$ and $\left[s_{i^{\prime}}^{*}, s_{i^{\prime}}^{*}+d \breve{c}_{i^{\prime}}\right]$ are disjoint for all $c \in B\left(c^{*}, \gamma\right)$, and, moreover, $\left[s_{i}^{*}, s_{i}^{*}+d \breve{c}_{i}\right] \cap J^{*}(j, \beta / 2)=\varnothing$ if $s_{i}^{*} \notin J^{*}(j, \beta / 2)$ $\left(J^{*}(j, \beta / 2)\right.$ is closed). Let $J_{d, c}^{\beta, j}$ be the set of points $t$ in $\left[\tau+d \breve{c}_{1}, \infty\right) \cap J^{*}(j, \beta / 2)$ for which

$$
\hat{g}_{x}^{j}(t) q^{* *}\left(t, d c, P^{*}\right) \geq d \alpha / 4 \text { for all } c \in B\left(c^{*}, \gamma\right)
$$

Now,

$$
\hat{J}_{d, c}^{\beta, j}:=\left[J^{*}(j, \beta / 2) \backslash\left(\bigcup_{i}\left[s_{i}^{*}, s_{i}^{*}+d \breve{c}_{i}\right)\right)\right] \cap[\tau, \infty) \subset J_{d, c}^{\beta, j}
$$

by (40) because $q^{* *}\left(t, d c, P^{*}\right)=q^{*}\left(t, d c, P^{*}\right)$ in $\hat{J}_{d, c}^{\beta, j}$. Let $a_{0}=1 / 2$ and define $a_{n}$ inductively by the for mula $a_{n}=a_{n-1}-\left(a_{n-1}\right)^{2}\left(a_{n} \downarrow 0\right)$. Let $b_{n}=a_{n-1}-a_{n}$, and, for $i=1, \cdots, i^{*}:=\max \left\{i: s_{i}=\tau\right\}$, let $M_{n, i}^{c}=\left(\tau+a_{n}+b_{n} \sum_{k=1}^{i-1} \hat{c}_{k}, \tau+a_{n}+b_{n} \sum_{k=1}^{i} \hat{c}_{k}\right], \quad M_{i}^{c}=\bigcup_{n \geq 1} M_{n, i}^{c}$ (see (37) for $\left.\hat{c}_{k}\right)$. Then, assuming right continuity at $\tau$, (see $\left.(9)^{7}\right)$, it is easily seen that

$$
\left|(1 / \eta)\left\{\int_{\tau}^{\tau+\eta} \sum_{i=1}^{i^{*}} 1_{M_{i}^{c}}(t) f\left(t, x^{*}(t), u_{i}^{*}\right) \mathrm{d} t-\eta \sum_{i=0}^{i^{*}} \hat{c}_{i} f\left(\tau, x^{*}(\tau), u_{i}^{*}\right)\right\}\right|
$$

is small when $\eta$ is small, $\eta>0$, uniformly in $c \in E_{m^{*}}$. See the arguments connected with (74) below. For $d \in\left(0, d_{1}\right], \quad$ let $\quad u\left(t, d c, P^{*}\right)=\breve{u}(t):=\sum_{i=1}^{i^{*}} 1_{M_{i}^{c}}(t) u_{i}^{*} \quad$ for $\quad t \in\left(\tau, \tau+d \breve{c}_{1}\right], \quad$ let $\quad u\left(t, d c, P^{*}\right)=u_{i}^{*} \quad$ for $t \in\left[s_{i}^{*}, s_{i}^{*}+d \breve{c}_{i}\right], \quad i>i^{*} \quad$ (recall that these intervals are disjoint when the left ends differ and that $\left.s_{i^{\prime}}^{*}=s_{i}^{*} \Rightarrow u_{i^{\prime}}^{*}=u_{i}^{*}\right)$, and $u\left(t, d c, P^{*}\right)=u^{*}(t)$ elsewhere. Let $x\left(t, d c, P^{*}\right)$ be the corresponding solution. Define

$$
\begin{aligned}
q\left(t, d c, P^{*}\right):= & \int_{0}^{t} C(t, s) 1_{\left[\tau, \tau+d \bar{c}_{1}\right]}(s)\left[f\left(s, x^{*}(s), \breve{u}(s)\right)-f^{*}(s)\right] \mathrm{d} s \\
& +\sum_{i>i^{*}} \int_{0}^{t} C(t, s) 1_{\left[s_{i}^{*}, s_{i}^{*}+d \bar{c}_{i}\right]}(s) \hat{c}_{i}\left[f\left(s, x^{*}(s), u_{i}^{*}\right)-f^{*}(s)\right] \mathrm{d} s \\
= & \int_{0}^{t} C(t, s)\left(f\left(s, x^{*}(s), u\left(s, d c, P^{*}\right)\right)-f^{*}(s)\right) \mathrm{d} s
\end{aligned}
$$

\footnotetext{
${ }^{7}$ Only when there are more than one perturbation $u_{i}$ at $\tau$ right continuity is needed to obtain (43). If there is a single perturbation at $\tau$, (i.e. for all $i=\left\{i: s_{i}=\tau\right\}, u_{i}=$ some $\bar{u}$ ) it suffices that $\tau$ is a right Lebesgue point of $f\left(t, x^{*}(t), \bar{u}\right)$. Finally, if $G(0+)=\varnothing$, then we shall throughout assume a single perturbation $\bar{u} \in U^{\prime}$ at $\tau$.
} 
Now, for $d$ small, $C(t, s) \simeq C\left(t, s_{i}^{*}\right)$ for $s \in\left(s_{i}^{*}, s_{i}^{*}+d \breve{c}_{i}\right]$, hence by (43) and the Lebesgue point property of $f^{*}($.$) and f\left(t, x^{*}(t), u_{i}^{*}\right)$ at $s_{i}^{*}, q\left(t, d c, P^{*}\right)-q^{* *}\left(t, d c, P^{*}\right)$ is of the second order in $d$ uniformly in $c \in E_{m^{*}}$ and $t \in\left[\tau, T^{\prime}\right]$. Let $\breve{J}_{d, c}^{\beta, j}=J_{d, c}^{\beta, j} \cup\{J(j, \beta / 2) \cap[\tau, \infty)\}$. We want to prove that for some $d_{*}>0$

$$
\text { for } c \in B\left(c^{*}, \gamma\right) \text { and } d \in\left(0, d_{*}\right], g^{j}\left(t, x\left(t, d c, P^{*}\right)\right) \geq 0 \text { for all } t \in \breve{J}_{d, c}^{\beta, j}
$$

Because $\hat{g}_{x}^{j}(t) C\left(t, T^{\prime}\right)$ is bounded by assumption (25), and $q\left(T^{\prime}, d c, P^{*}\right)-q^{* *}\left(T^{\prime}, d c, P^{*}\right)$ is of the second order, $\hat{g}_{x}^{j}(t) C\left(t, T^{\prime}\right)\left(q\left(T^{\prime}, d c, P^{*}\right)-q^{* *}\left(T^{\prime}, d c, P^{*}\right)\right)=\hat{g}_{x}^{j}(t)\left(q\left(t, d c, P^{*}\right)-q^{* *}\left(t, d c, P^{*}\right)\right)$ is of the second order, uniformly in $t \in\left[T^{\prime}, \infty\right)$. Moreover, $\hat{g}_{x}^{j}(t)\left(q\left(t, d c, P^{*}\right)-q^{* *}\left(t, d c, P^{*}\right)\right)$ is of the second order, uniformly in $t \in\left[\tau+d \breve{c}_{1}, T^{\prime}\right)$, by the boundedness of $\sup _{t \in\left[0, T^{\prime}\right]} \hat{g}_{x}^{j}(t)$. Hence, by (41), for some positive $d_{2} \leq d_{1}$, for $d \in\left(0, d_{2}\right]$

$$
g_{x}^{j}\left(t, x^{*}(t)\right) q\left(t, d c, P^{*}\right) \geq d \alpha / 8 \text { for } t \in J_{d, c}^{\beta, j} \text { for all } c \in B\left(c^{*}, \gamma\right)
$$

Next, it is well-known that

$$
x\left(t, d c, P^{*}\right)-x^{*}(t)-q\left(t, d c, P^{*}\right) \text { is of the second order in } d
$$

uniformly in $t \in\left[\tau, T^{\prime}\right], \quad c \in E_{m^{*}}$ (see Lemma D in Appendix). Moreover, by the differentiability assumption on $x \rightarrow g^{j}$ at $\left(t, x^{*}(t)\right)((28))$, for some positive second order term $o_{*}(d)$, we have

$$
g^{j}\left(t, x\left(t, d c, P^{*}\right)\right)-g^{j}\left(t, x^{*}(t)\right)-\hat{g}_{x}^{j}(t)\left[x\left(t, d c, P^{*}\right)-x^{*}(t)\right] \geq-o_{*}(d)
$$

for all $t \in\left[\tau, T^{\prime}\right]$ (because $x\left(., d c, P^{*}\right)-x^{*}($.$\left.) is of the first order in \left[\tau, T^{\prime}\right]\right)$. Hence, for some positive second order term $o^{*}(d)$, for $c \in B\left(c^{*}, \gamma\right)$

$$
g^{j}\left(t, x\left(t, d c, P^{*}\right)\right)-g^{j}\left(t, x^{*}(t)\right)-g_{x}^{j}\left(t, x^{*}(t)\right) q\left(t, d c, P^{*}\right) \geq-o^{*}(d)
$$

both for all $t \in\left[\tau, T^{\prime}\right]$ by boundedness of $\hat{g}_{x}^{j}$ on $\left[\tau, T^{\prime}\right]$ (combine (46) and (47)) and for all $t \geq T^{\prime}$ (combine (27) and (26)). Then, by (45), for some positive $d_{3} \leq d_{2}$,

$g^{j}\left(t, x\left(t, d c, P^{*}\right)\right) \geq g^{j}\left(t, x^{*}(t)\right)+d \alpha / 8-o^{*}(d) \geq d \alpha / 16$, for $d \in\left(0, d_{3}\right], \quad c \in B\left(c^{*}, \gamma\right), \quad t \in J_{d, c}^{\beta, j}$. Moreover, for some positive $d_{4} \leq d_{3}$, for $d \in\left(0, d_{4}\right]$, for all $t$, by (48),

$g^{j}\left(t, x\left(t, d c, P^{*}\right)\right)-g^{j}\left(t, x^{*}(t)\right)-g_{x}^{j}\left(t, x^{*}(t)\right) q\left(t, d c, P^{*}\right) \geq-\beta / 4$ and (by (25)),

$\left|g_{x}^{j}\left(t, x^{*}(t)\right) q\left(t, d c, P^{*}\right)\right| \leq \beta / 4$ uniformly in $t \in[\tau, \infty), \quad c \in E_{m^{*}}$, so uniformly for $t \in J(j, \beta / 2), \quad t \geq \tau$, $c \in E_{m^{*}}$

$$
\begin{aligned}
g^{j}\left(t, x\left(t, d c, P^{*}\right)\right) & \geq g^{j}\left(t, x^{*}(t)\right)+g_{x}^{j}\left(t, x^{*}(t)\right) q\left(t, d c, P^{*}\right)-\beta / 4 \\
& \geq \beta / 2-\beta / 4+g_{x}^{j}\left(t, x^{*}(t)\right) q\left(t, d c, P^{*}\right) \\
& \geq \beta / 2-\beta / 4-\beta / 4=0
\end{aligned}
$$

Hence, (44) has been shown, and in particular, (see (42))

$$
\text { (44) holds for } t \in\left\{\left[J^{*}(j, \beta / 2) \backslash\left(\bigcup_{i}\left[s_{i}^{*}, s_{i}^{*}+d \breve{c}_{i}\right]\right)\right] \cup J(j, \beta / 2)\right\} \cap[\tau, \infty)
$$

So far, we have only used the basic assumptions and the first of the three conditions on $g$ in Remark 3, namely (28). The other two properties, (30) and (29), will be used in what follows. 
We want to show that $\left[s_{i}^{*}, s_{i}^{*}+d \breve{c}_{i}\right] \cap J^{*}(j, \beta / 2) \subset J_{d, c}^{\beta, j}$ when $s_{i}^{*} \in J^{*}(j, \beta / 2), \quad s_{i}^{*}>\tau, \quad c \in B\left(c^{*}, \gamma\right)$. Now, $s_{i}^{*} \in J^{*}(j, \beta / 2)$ implies $s \in J^{*}(j, \beta)$ for $s<s_{i}^{*}, s$ close to $s_{i}^{*} \quad$ (recall $s_{i}^{*} \notin\left\{a_{1}, \cdots, a_{j^{*}}\right\}$ ). Thus, by (40), $\hat{g}_{x}^{j}(t) q^{*}\left(t, d c, P^{*}\right) \geq d \alpha / 2$ for $t \in\left[s_{i}^{*}, s_{i}^{*}+d \breve{c}_{i}\right] \cap J^{*}(j, \beta / 2)$ and $\hat{g}_{x}^{j}\left(s_{i}^{*}\right) q_{*}\left(s_{i}^{*}, d c, P^{*}\right)=\hat{g}_{x}^{j}\left(s_{i}^{*}\right) q^{*}\left(s_{i}^{*}-, d c, P^{*}\right) \geq d \alpha / 2, \quad c \in B\left(c^{*}, \gamma\right)$. For $d$ small $\left(d \in\left(0, d_{5}\right], d_{5} \leq d_{4}\right)$, $\hat{g}_{x}^{j}(t) q_{*}\left(t, d c^{*}, P^{*}\right) \geq d \alpha / 4$ when $t \in\left[s_{i}^{*}, s_{i}^{*}+d \breve{c}_{i}\right], \quad c \in B\left(c^{*}, \gamma\right)$ (by continuity,

$\hat{g}_{x}^{i}(t) C\left(t, s_{k}^{*}\right)-\hat{g}_{x}^{j}\left(s_{i}^{*}\right) C\left(s_{i}^{*}, s_{k}^{*}\right), \quad k<i$, is small, when $t-s_{i}^{*}$ is small). Note that if $t \in\left[s_{i}, s_{i}+d \breve{c}_{i}\right)$ for some $i, q^{* *}\left(t, d c, P^{*}\right)$ is a convex combination of $q^{*}(t, d c, P)$ and $q_{*}(t, d c, P)$ (the former one has weight $\left.\left(t-s_{i}\right) / d \breve{c}_{i}\right)$. Hence, for any $t \in\left[s_{i}^{*}, s_{i}^{*}+d \breve{c}_{i}\right), q^{* *}\left(t, d c, P^{*}\right)$ belongs to the segment between $q^{*}\left(t, d c, P^{*}\right)$ and $q_{*}\left(t, d c, P^{*}\right)$, hence $\hat{g}_{x}^{j}(t) q^{* *}\left(t, d c, P^{*}\right) \geq d \alpha / 4$ for $t \in\left[s_{i}^{*}, s_{i}^{*}+d \breve{c}_{i}\right) \cap J^{*}(j, \beta / 2), d \in\left(0, d_{5}\right]$, $c \in B\left(c^{*}, \gamma\right)$.

So far, we have proved (44) for $t \in\left\{J^{*}(j, \beta / 2) \cap\left[\tau+d \breve{c}_{1}, \infty\right)\right\} \cup\{J(j, \beta / 2) \cap[\tau, \infty)\}$, recall that if $s_{i}^{*} \notin J^{*}(j, \beta / 2)$, then for $d \in\left(0, d_{1}\right],\left[s_{i}^{*}, s_{i}^{*}+d \breve{c}_{i}\right] \cap J^{*}(j, \beta / 2)=\varnothing \subset J_{d, c}^{\beta, j}$ (in particular this holds for $s_{i}^{*}=\tau$, so for $\tau \notin J^{*}(j, \beta / 2)$, (44) holds for all $\left.t \geq \tau\right)$.

Finally, let us prove (44) for $t \in\left[\tau, \tau+d \breve{c}_{1}\right], \quad \tau \in J^{*}(j, \beta / 2)$. We can assume that $\beta>0$ is so small that $g^{j}\left(\tau, x^{*}(\tau)\right)>\beta$, if $g^{j}\left(\tau, x^{*}(\tau)\right)>0, \tau \in J^{j}$. So consider the case where $g^{j}\left(\tau, x^{*}(\tau)\right)=0, \tau \in J^{j}$.

Then the right derivative $\left[\mathrm{d}^{+} g^{j}\left(t, x^{*}(t)\right) / \mathrm{d} t\right]_{t=\tau}=g_{x}^{j}\left(\tau, x^{*}(\tau)\right) f^{*}(\tau)+g_{t}^{j}\left(\tau, x^{*}(\tau)\right) \geq 0 \quad\left(\tau \notin\left\{b_{j}\right\}_{j}\right)$. First consider the subcase where $i^{*}=1$ and $\tau=s_{1}^{*}$ (only one $s_{i}^{*}=\tau$ ). By (39), $\hat{g}_{x}^{j}(\tau)\left[\breve{c}_{1}\left(f\left(\tau, x^{*}(\tau), u_{1}^{*}\right)-f^{*}(\tau)\right)\right] \geq \alpha / 2, \quad c \in B\left(c^{*}, \gamma\right)$. Combining the two last weak inequalities we get $\breve{c}_{1}\left[\hat{g}_{x}^{j}(\tau) f\left(\tau, x^{*}(\tau), u_{1}^{*}\right)+g_{t}^{j}\left(\tau, x^{*}(\tau)\right)\right] \geq \alpha / 2$. Then $\left[\mathrm{d}^{+} g^{j}\left(t, x\left(t, d c, P^{*}\right)\right) / \mathrm{d} t\right]_{t=\tau} \geq \alpha / 2 \breve{c}_{1} \geq \alpha / 2$, so $g^{j}\left(t, x\left(t, d c, P^{*}\right)\right) \geq 0$ for $t \in\left[\tau, \tau+d \breve{c}_{1}\right], \quad c \in B\left(c^{*}, \gamma\right)$, for $d$ small $\left(d \in\left(0, d_{6}\right], d_{6} \leq d_{5}\right)$.

Consider next the subcase where $P^{*}$ contains several pairs $\left(\tau, u_{i}^{*}\right), \quad i=1, \cdots, i^{*}, \quad u_{i}^{*} \in U, \quad \tau=s_{1}^{*}=\cdots=s_{i^{*}}^{*}$, $i^{*}>1$. Using (43), it is easily seen that $\left[\left(\mathrm{d}^{+} / \mathrm{d} s\right) g^{j}\left(s, x\left(s, d c, P^{*}\right)\right)\right]_{s=\tau}=g_{x}^{j}\left(\tau, x^{*}(\tau)\right) \sum_{i=1}^{i^{*}} \hat{c}_{i} f\left(\tau, x^{*}(\tau), u_{i}^{*}\right)+g_{t}^{j}\left(\tau, x^{*}(\tau)\right)$. When $\tau \in\left[a_{j}, b_{j}\right)$ and $g^{j}\left(\tau, x^{*}(\tau)\right)=0$, we have that $\left.\left[\mathrm{d}^{+} g^{j}\left(t, x^{*}(t)\right) / \mathrm{d} t\right]\right]_{\tau=\tau}=\hat{g}_{x}^{j}(\tau) f^{*}(\tau)+g_{t}^{j}\left(t, x^{*}(\tau)\right) \geq 0$. Now, $\hat{g}_{x}^{j}(\tau)\left[\sum_{i=1}^{i^{*}} c_{i}\left\{f\left(\tau, x^{*}(\tau), u_{i}^{*}\right)-f^{*}(\tau)\right\}\right] \geq \alpha / 2$ by (39), so $\alpha / 2 \breve{c}_{1} \leq \hat{g}_{x}^{j}(\tau)\left[\sum_{i=1}^{i^{*}} \hat{c}_{i} f\left(\tau, x^{*}(\tau), u_{i}^{*}\right)+g_{t}^{j}\left(\tau, x^{*}(\tau)\right)\right]=\left[\left(\mathrm{d}^{+} / \mathrm{d} s\right) g^{j}\left(s, x\left(s, d c, P^{*}\right)\right)\right]_{s=\tau}$. Hence we again get $g^{j}\left(t, x\left(t, d c, P^{*}\right)\right) \geq 0$ for $t \in\left[\tau, \tau+d \breve{c}_{1}\right], \quad c \in B\left(c^{*}, \gamma\right)$, for $d$ small $\left(d \in\left(0, d_{6}\right], \quad d_{6} \leq d_{5}\right)$.

Thus, when $c^{*}, P^{*}$ satisfies (38), then

$$
\text { for } c \in B\left(c^{*}, \gamma\right), d \in\left(0, d_{6}\right], g^{j}\left(t, x\left(t, d c, P^{*}\right)\right) \geq 0 \text { for all } t \in J^{j}, t \geq \tau
$$

\subsection{Local Controllability at Infinity}

Observation 1. Define $\pi:=\left(x_{1}, \cdots, x_{n}\right) \rightarrow\left(x_{1}, \cdots, x_{n^{n}}\right)$ and $\tilde{Q}:=\left\{\tilde{q} \in \mathbb{R}^{n^{n}}: \tilde{q}_{i}=0\right.$ for $i \leq n^{\prime}, \tilde{q}_{i} \in[-1,1]$ for 
all $i=n^{\prime}+1, \cdots, n^{\prime \prime}$, with $\tilde{q}^{i} \leq 0$ if $\left.x^{* i}(\infty)=\bar{x}^{i}\right\}$. Note that by Lemma B in Appendix, for all $c$ and $P$, $\pi q^{*}(\infty, c, P)$ and $\pi x(\infty, c, P)$ exist. Let $K \subset \mathbb{R}^{n^{\prime \prime}}$ be the convex set $\left\{\tilde{q}+\pi q^{*}(\infty, c, P): \tilde{q} \in \tilde{Q}, q^{*}(., c, P) \in Q_{\tau}^{\prime}\right\} \quad$ (for $Q_{\tau}^{\prime}$ see (38)). If $y \in \operatorname{int} K$, then for some positive $\delta$, some $y_{i} \in \mathbb{R}^{n^{\prime \prime}}, \quad i=1, \cdots, n^{\prime \prime}+1, \quad B(y, \delta) \subset$ int $\operatorname{co}\left\{y_{1}, \cdots, y_{n^{\prime \prime}+1}\right\}$, where each $y_{i}$ equals $q^{i}+\pi q^{*}\left(\infty, c^{i}, P_{i}\right)$, $q^{i} \in \tilde{Q}, \quad c^{i} \in E_{m^{i}}, P_{i}=\left(\left(s_{1}^{i}, u_{1}^{i}\right), \cdots,\left(s_{m^{i}}^{i}, u_{m^{i}}^{i}\right)\right) \in D_{m^{i}}^{\tau}, \quad q^{*}\left(., c^{i}, P_{i}\right) \in Q_{\tau}^{\prime}, \quad y^{1}-y^{n^{n+1}+1}, \cdots, y^{n^{\prime \prime}}-y^{n^{n+1}+1}$ linearly independent. Let $\Lambda_{n^{\prime \prime}+1}:=\left\{\lambda: \sum_{i=1}^{n^{n}+1} \lambda_{i}=1, \lambda_{i}>0\right\}$. Then $B(y, \delta) \subset\left\{\sum \lambda_{i}\left[q_{i}+\pi q^{*}\left(\infty, c^{i}, P_{i}\right)\right]: \lambda \in \Lambda_{n^{\prime \prime}+1}\right\}$, $y=\sum \lambda_{i}^{*}\left[q_{i}+\pi q^{*}\left(\infty, c^{i}, P_{i}\right)\right]$ for some vector $\lambda^{*} \in \Lambda_{n^{*}+1}$. In fact, for each $z \in B(y, \delta)$ there is a unique $\lambda(z) \in \Lambda_{n^{\prime \prime}+1}$ such that $z=\sum \lambda_{i}(z) y_{i}, \quad \lambda(z)$ evidently depends linearly on $z$, note that $z \rightarrow \lambda(z)$ extends linearly to all $\mathbb{R}^{n^{*}+1}$. Let $P_{i j}=\left(s_{j}^{i}, u_{j}^{i}\right)$, and let $P^{*}$ consist of all $\left(s_{j}^{i}, u_{j}^{i}\right), \quad j=1, \cdots, m^{i}, \quad i=1, \cdots, n^{\prime \prime}+1$, for the moment we allow the pairs in $P^{*}$ to be doubly indexed, (and the time points not to be ordered). Then $\sum \lambda_{i}(z) q^{*}\left(., c^{i}, P_{i}\right)=\sum_{i} \sum_{j=1}^{m^{i}} \lambda_{i}(z) c_{j}^{i} q^{*}\left(., 1, P_{i j}\right)=q^{*}\left(., c(z), P^{*}\right)$, where $c_{i, j}(z)=\lambda_{i}(z) c_{j}^{i}$ (as we have double indices on $P^{*}$, we have double indices on the components of $\left.c(z)\right)$. Note that $c_{i, j}(z)>0$,

$\sum_{i} \sum_{j=1}^{m^{i}} c_{i, j}(z)<1$. Of course, we can re-index the pairs in $P^{*}$ (and so also the entities $c_{i j}(z)$ ) by using a single index, with the time points in the pairs in increasing order. Let $m^{*}$ be the number of pairs $(i, j)$. We use $P^{*}$ also as the name of the vector of reindexed pairs, and $c(z) \in E_{m^{*}}$ for the vector consisting of all entities $c_{i j}(z)$, reindexed in the same manner as the pairs in $P^{*}$. Then for ${ }^{m} z \in B(y, \delta)$, $z=\sum_{i} \lambda_{i}(z) q^{i}+\pi q^{*}\left(\infty, c(z), P^{*}\right), \quad q^{*}\left(., c(z), P^{*}\right) \in Q_{\tau}^{\prime}, \quad z \mapsto(\lambda(z), c(z))$ linear.

The following result should surprise nobody, a proof however is given in Appendix.

Lemma 1. Assume that for $z \in B(y, \delta), \quad z=\sum_{i} \lambda_{i}(z) q^{i}+\pi q^{*}\left(\infty, c(z), P^{*}\right), \quad z \mapsto(\lambda(z), c(z))$ linear, $\lambda(z) \in \Lambda_{n^{*}+1}, \quad c(z) \in E_{m^{*}}$. Then there exist some first order term $O(d)$ (i.e. $\lim _{d \downarrow 0} O(d)=0$ ) and some $\rho>0$ such that for each $d \in(0, \rho]$ for some $c=c^{d} \in E_{m^{*}}$, some $q^{d} \in \operatorname{co}\left\{q^{1}, \cdots, q^{n^{n+1}}\right\}$, $d q^{d}+\pi x\left(\infty, d c^{d}, P^{*}\right)-\pi x^{*}(\infty)=d y,\left|c^{d}-c(y)\right| \leq O(d), \quad\left|q^{d}(d)-\sum \lambda_{i}(y) q^{i}\right| \leq O(d)$.

\subsection{Separation Arguments That Yield the Multipliers}

By optimality, for all $\delta^{*}>0, \quad z^{*}:=\left(\delta^{*}, 0, \cdots, 0\right) \notin$ int $K$. To see this, assume, by contradiction the opposite, that for some $\delta^{*}>0, \quad z^{*}:=\left(\delta^{*}, 0, \cdots, 0\right) \in \operatorname{int} K$. Then by Observation 1 and Lemma 1 , for $d>0$ and small, for some $q^{d} \in \tilde{Q}, \quad c^{d} \in E_{m^{*}}, \quad d z^{*}+\pi x^{*}(\infty)=d q^{d}+\pi x\left(\infty, d c^{d}, P^{*}\right)$ and $x\left(., d c^{d}, P^{*}\right)$ satisfies the unilateral time constraint (3), see (51) (because $q^{*}\left(., c(y), P^{*}\right) \in Q_{\tau}^{\prime}$ and $\left|c^{d}-c(y)\right| \leq O(d)$ ). The last equality gives that $x\left(\infty, d c^{d}, P^{*}\right)$ satisfies the terminal constraint (4) for $d$ small, and that $a x^{*}(\infty)<d \delta^{*}+a x^{*}(\infty)=d a z^{*}+a x^{*}(\infty)=a x\left(\infty, d c^{d}, P^{*}\right)$. This contradicts the optimality of $x^{*}($.$) . Thus the$ sets int $K$ and $L_{*}:=\left\{\left(\delta^{*}, 0, \cdots, 0\right) \in \mathbb{R}^{n^{n}}: \delta^{*}>0\right\}$ are disjoint (this is trivial if int $K=\varnothing$ ). Thus these sets can be separated ${ }^{8}$ : there exists a nonzero vector $p^{*} \in \mathbb{R}^{n^{\prime}}$, such that $p^{*} K \leq 0 \leq p^{*} L_{*}$. As $\tilde{Q} \subset K$, this inequality gives that

$$
p^{* i} \geq 0 \text { for } i \in\left\{1, n^{\prime}+1, \cdots, n^{\prime \prime}\right\}, p^{* i}=0 \text { if } x^{* i}(\infty)>\bar{x}^{i}, i>n^{\prime}
$$

${ }^{8}$ If int $K \neq \varnothing$ and $\operatorname{int} K \cap L_{*}=\varnothing$, then $K$ and $L_{*}$ can be separated. If $\operatorname{int} K=\varnothing$, then for some nonzero $p^{*}, p^{*} K=0$, and $p^{*}$ can be chosen such that $p^{*} L_{*} \geq 0$. 
Define $L:=\left\{h(.) \in L_{\infty}\left([\tau, \infty), \mathbb{R}^{j *}\right):\right.$ for all $j$, for some positive $\alpha, \beta, h^{j}(t)>\alpha$ when $\left.t \in J^{*}(j, \beta), t \geq \tau\right\}$. Recall that $\sup _{t}\left|g_{x}^{j}\left(t, x^{*}(t)\right) q^{*}(t, c, P)\right|<\infty$ for all $c, P$, see (25), and write $g=\left(g^{1}, \cdots, g^{j *}\right)$. Note that $L^{\prime}:=\left\{\left(g_{x}\left(., x^{*}().\right) q^{*}(., c, P), p^{*} \pi q^{*}(\infty, c, P)\right): q^{*}(.,, c, P) \in Q_{\tau}^{\prime}\right\}$ (for $Q_{\tau}^{\prime}$, see (38)) has to be disjoint from the convex body $L \times(0, \infty)$ in $L_{\infty}\left([\tau, \infty), \mathbb{R}^{j *}\right) \times \mathbb{R}$, otherwise the inequality $p^{*} K \leq 0$ is contradicted. By separation, for some continuous linear functional $\psi:=\left(\psi_{1}, \cdots, \psi_{j}^{*}\right)$ on $L_{\infty}\left([\tau, \infty), \mathbb{R}^{j *}\right)$ and some number $\lambda, \quad(\lambda, \psi) \neq 0$

$$
\psi g_{x}\left(., x^{*}(.)\right) q^{*}(., c, P)+\lambda p^{*} \pi q^{*}(\infty, c, P) \leq \psi h(.)+\lambda h^{\prime}
$$

for all $\left(h(),. h^{\prime}\right) \in L \times(0, \infty)$, all $q^{*}(., c, P) \in Q_{\tau}^{\prime}$. Evidently, by this inequality, $\lambda \geq 0$ and $\psi$ are nonnegative on $L$, with $\psi_{j}\left(1_{[\tau, \infty) \cap \subset J^{*}(j, \beta)}\right)=0$ for all $\beta>0$. Each $\psi_{j}$ can be represented by a bounded finitely additive nonnegative set function $\mu_{j}$ vanishing on sets of Lebesgue measure zero. Evidently, $\mu_{j}$ vanishes on $[\tau, \infty) \cap \complement J^{*}(j, \beta)$ for all $\beta>0$, in particular $\mu_{j}$ vanishes on $[\tau, \infty) \cap \complement\left[a_{j}, b_{j}\right]$. The inequality (53) gives that, for $\left.p_{\infty}=\left(\lambda p^{*}, 0\right) \in \mathbb{R}^{n^{\prime}} \times \mathbb{R}^{n-n^{\prime}}\right)$, for all pairs $(s, u), \quad u \in U, \quad s=\tau, \quad\left(\tau\right.$ still fixed in $\left.J^{\prime}\right)$ and for all pairs $(s, u), \quad s>\tau, \quad s \in J_{u}$ and $u \in U^{\prime}$

$$
p_{\infty} C(\infty, s) v(s)+\sum_{j} \int_{[s, \infty)} g_{x}^{j}\left(t, x^{*}(t)\right) C(t, s) v(s) \mathrm{d} \mu_{j}(t) \leq 0
$$

where $v(s)=v^{u}(s):=f\left(s, x^{*}(s), u\right)-f^{*}(s)$ (To obtain (54), in (53) let $q^{*}(., c, P)=C(., . s) v(s)$ ). Moreover, (54) also holds for $\tau$, for $v(\tau)=\sum_{i=1}^{i_{\tau}} \lambda_{i}^{\tau}\left(f\left(\tau, x^{*}(\tau), u_{i}^{\tau}\right)-f^{*}(\tau)\right)$, for any given $u_{i}^{\tau} \in U, \lambda^{\tau} \in E_{i_{\tau}}$.

Let us now choose a sequence $\tau_{n} \in J^{\prime}$ converging to zero when $n \rightarrow \infty$ such that (54) holds in the manner described for $\tau=\tau_{n}$ for certain multipliers $p_{\infty}=p_{\infty}^{n}, \quad \mu=\mu^{n}=\left(\mu_{1}^{n}, \cdots, \mu_{j^{*}}^{n}\right)$. In particular (54) holds for $s=\tau_{n}$ for any given $v(\tau)=v\left(\tau_{n}\right)$. Let us fix such a sequence $v\left(\tau_{n}\right)$, assuming it to be bounded. We can assume that $\left|p_{\infty}^{n}\right|+\sum_{j} \mu_{j}^{n}([0, \infty))=1$ (we extend $\mu^{n}$ to $[0, \infty)$ by letting $\mu^{n}([0, \tau))=0$ ). Using the weak* topology on $\left\{\mu^{n}\right\}_{n}$, there exists a cluster point $\left(p_{\infty}, v_{0}, \mu\right)$ of the sequence $\left(p_{\infty}^{n}, v\left(\tau_{n}\right), \mu^{n}\right)$ satisfying

$$
\text { (52), } \mu \geq 0, \mu_{j}\left(\complement^{*}(j, \beta)\right)=0 \text { for all } \beta>0,\left|p_{\infty}\right|+\sum \mu_{j}([0, \infty))=1
$$

(for some subsequence $n_{k}, \quad\left(p_{\infty}^{n}, \mu^{n_{k}}([0, \infty))\right) \rightarrow\left(p_{\infty}, \mu([0, \infty))\right)$ ). (by the cluster point property, so the last equality holds). The cluster point $\mu$ is a bounded nonnegative finitely additive set function that vanishes on Lebesgue null sets. It is furthermore easily seen that (54) holds for $p_{\infty}$ and $\mu$, for $u \in U^{\prime}, s \in J_{u}$ and for $s=0$, both for $v(0)$ equal to the cluster point $v_{0}{ }^{9}$ as well as for $v(0)=\lim _{n} \breve{v}\left(\tau_{n}\right)$, provided this limit exists, for any $\breve{v}\left(\tau_{n}\right)=\sum_{i=1}^{i_{n}} \breve{\lambda}_{i}^{\tau_{n}}\left(f\left(\tau_{n}, x^{*}\left(\tau_{n}\right), \breve{u}_{i}^{\tau_{n}}\right)-f^{*}\left(\tau_{n}\right)\right)$, for any given $\breve{u}_{i}^{\tau_{n}} \in U, \quad \breve{\lambda}^{\tau_{n}} \in E_{i_{\tau_{n}}}$.

\footnotetext{
${ }^{9}$ For simplicity, assume that $v\left(\tau_{n}\right) \rightarrow v_{0}$. Then, for any $\varepsilon>0$, for all $n$ large enough, $\left|C\left(t, \tau_{n}\right) v\left(\tau_{n}\right) 1_{\left[\tau_{n}, T\right]}-C(t, 0) v_{0} 1_{\left\{\tau_{n}, T\right]}\right| \leq \varepsilon$ for all for $t \in\left[\tau_{n}, T^{\prime}\right]$, so, by (25); for $\Psi_{n}(t):=g_{x}\left(t, x^{*}(t)\right) C\left(t, \tau_{n}\right) v\left(\tau_{n}\right), \Psi:=g_{x}\left(t, x^{*}(t)\right) C(t, 0) v_{0}$, for any $\varepsilon^{\prime}>0$; for all $n$ large enough, $\left|\Psi_{n}(t) 1_{\tau_{\left.\tau_{n}, \infty\right)}}-\Psi 1_{\tau_{\left.\tau_{n}, \infty\right)}}\right| \leq \varepsilon^{\prime}$ for all $t \in\left[\tau_{n}, \infty\right)$. As $\left|\mu^{n}\right| \leq 1,\left|\int_{\left[\tau_{n}, \infty\right)}\left(\Psi_{n}(t)-\Psi(t)\right) \mathrm{d} \mu^{n}(t)\right| \leq \operatorname{essup}_{t z \tau_{n}}\left|\Psi_{n}(t)-\Psi(t)\right|$, so $\int_{\left[\tau_{n}, \infty\right)} \Psi_{n}(t) \mathrm{d} \mu^{n}(t)-\int_{[0, \infty)} \Psi(t) \mathrm{d} \mu(t)=\int_{\left[n_{n}, \infty\right)}\left(\Psi_{n}(t)-\Psi(t)\right) \mathrm{d} \mu^{n}(t)+\int_{\left[n_{n}, \infty\right)}^{\infty} \Psi_{n}(t) \mathrm{d} \mu^{n}(t)-\int_{[0, \infty)} \Psi(t) \mathrm{d} \mu(t)$, which converges to zero at least for a subsequence of $\mu^{n}$.
} 
Now $\hat{\mu}^{j}(s):=\int_{[0, s]} \mathrm{d} \mu_{j}(t)$ is nondecreasing and bounded. Let $J_{0}$ be the continuity points in $(0, \infty)$ of $s \rightarrow \int_{[0, s]} \mathrm{d} \mu(t), \quad \mu:=\left(\mu_{1}, \cdots, \mu_{j^{*}}\right)$. For any $u \in U^{\prime}$ and for any $s \in J_{0} \cap J^{\prime \prime}$, a sequence $s_{n} \in J_{u}$ exists, such that $s_{n} \rightarrow s$, and $v^{u}\left(s_{n}\right) \rightarrow v^{u}(s)$ (see the very beginning of this proof), and because (54) holds for $\left(s_{n}, v^{u}\left(s_{n}\right)\right)$, it is easily seen by taking limits that (54) holds for $u$ and $s \in J_{0} \cap J^{\prime \prime}{ }^{10}$.

Finally, let us extract an additional property. If $g^{j}\left(t, x^{*}(t)\right)>0$ for all $t$ in some interval $\left(0, \varepsilon_{j}\right]$ and $a_{j}=0$, then, for some $\delta_{n}>0, g^{j}\left(t, x^{*}(t)\right) \geq \delta_{n}$ for $t \in\left[\tau_{n}, \varepsilon_{j}\right]$ when $\tau_{n}<\varepsilon_{j}$, which by (55) implies $0=\mu_{j}^{n}\left(\left[\tau_{n}, \varepsilon_{j}\right]\right)=\mu_{j}^{n}\left(\left[0, \varepsilon_{j}\right]\right)$ and hence $\mu_{j}\left(\left[0, \varepsilon_{j}\right]\right)=0$. Thus

$$
j \notin G(0+) \Rightarrow \mu_{j}\left(\left[0, \varsigma_{j}\right]\right)=0
$$

for (say) $\varsigma_{j}=(1 / 2) \sup \left\{t: g^{j}\left(s, x^{*}(s)\right)>0\right.$ for $\left.s \in(0, t)\right\}>0^{11}$.

\subsection{Further Information on the Multipliers in Special Cases}

Let us prove the results concerning the multipliers in the three last sentences in Theorem 2 in the case where $a x(\infty)$ is maximized.

Define

$$
p(s)=p_{\infty} C(\infty, s)+\sum_{j} \int_{[s, \infty)} g_{x}^{j}\left(t, x^{*}(t)\right) C(t, s) \mathrm{d} \mu_{j}(t)
$$

Now, assume (9) and (10) $(\alpha)$, with $G(0+)=\{j\}$. We may assume of the sequence $\tau_{n}$ used above that $g_{x}^{j}\left(\tau_{n}, x^{*}\left(\tau_{n}\right)\right) f^{*}\left(\tau_{n}\right)+g_{t}^{j}\left(\tau_{n}, x^{*}\left(\tau_{n}\right)\right) \leq 0$ for the single $j \in G(0+)$ (there exists a sequence $t_{n} \downarrow 0$, such that $\left.g^{j}\left(t_{n}, x^{*}\left(t_{n}\right)\right)=0\right)$. Here (29) was used. Let $v^{*}:=\sum \lambda_{i} f\left(0, x^{*}(0), \tilde{u}_{i}\right)$ satisfy the inequality in (9), define $v_{*}(t):=\sum \lambda_{i} f\left(t, x^{*}(t), \tilde{u}_{i}\right)$, and assume that $v(t)=v_{*}(t)-f^{*}(t)$. Then, by (30), for some $\kappa>0$, for $\tau_{n}$ close to $0, g_{x}^{j}\left(\tau_{n}, x^{*}\left(\tau_{n}\right)\right) v_{*}\left(\tau_{n}\right)+g_{t}\left(\tau_{n}, x^{*}\left(\tau_{n}\right)\right) \geq \kappa$, which combined with the previous inequality gives $g_{x}^{j}\left(\tau_{n}, x^{*}\left(\tau_{n}\right)\right) v\left(\tau_{n}\right) \geq \kappa$. So $\hat{g}_{x}^{j}(0) v_{0} \geq \kappa, \quad v_{0}$ any given cluster point of $v\left(\tau_{n}\right)$. We can assume that (54) holds for this cluster point $v_{0}$, for $s=0$.

Evidently, $\hat{g}_{x}^{j}(t) C(t, 0) v_{0} \geq \kappa / 2$ for all $t$ close to 0 . Now, if $p_{\infty}=0$ and $\mu([s, \infty))=0$ for all $s>0$, then for any $s>0, \quad p(0)=\int_{[0, s)} \hat{g}_{x}^{j}(t) C(t, 0) \mathrm{d} \mu_{j}(t)$. From $p(0) v_{0} \leq 0$ we get, for all $s$ close to zero, that $0 \geq \int_{[0, s)} \hat{g}_{x}^{j}(t) C(t, 0) v_{0} \mathrm{~d} \mu_{j}(t) \geq \int_{[0, s)}(\kappa / 2) \mathrm{d} \mu_{j}$, which gives $\mu_{j}([0, s))=0$ for $s$ close to zero, and so

${ }^{10}$ If $U$ is replaced by $\tilde{U}(t)$ as in Remark 3, we asume that $s_{i} \notin\left\{b^{k}\right\}_{k}$. The perturbations $u(t, d c, P)$ will belong to $\tilde{U}(t)$, because in the proof we now require the $u_{i}$ 's to belong to $\breve{U}\left(s_{i}\right)$ and then $u_{i} \in \breve{U}(t)$ for $t$ in $\left[s_{i}, s_{i}+d \check{c}_{i}\right]$, for $d$ small. Then (54) holds for a.e. $s$ for $u \in \breve{U}(s) \quad\left(\Rightarrow u \in \breve{U}\left(s_{n}\right)\right.$ for $\left.n\right)$ and also for $s=0$, for any given cluster point $v_{0}$ of any given bounded sequence $v\left(\tau_{n}\right)=\sum_{i} \lambda_{i}^{\tau_{n}}\left(f\left(\tau_{n}, x^{*}\left(\tau_{n}\right), u_{i}^{\tau_{n}}\right)-f^{*}\left(\tau_{n}\right)\right)$ where $u_{i}^{\tau_{n}} \in \tilde{U}\left(\tau_{n}\right), \quad \tau_{n} \in J^{\prime}, \quad \lambda^{\tau_{n}} \in E_{i_{i_{n}}} \quad$ In addition, (54) holds for $(s, v(0))$ for all limits $v(0)$ of all such sequences $v\left(\tau_{n}\right)$ that are convergent. In particular, (54) holds for a given cluster point $v_{0}$ of a given sequence $v\left(\tau_{n}\right)$, where $v(t)=\sum_{i=1}^{i} \lambda_{i}\left(f\left(t, x^{*}(t), u_{i}\right)-f^{*}(t)\right), \quad u_{i}$ given points in $\breve{U}(0), \lambda \in E_{i_{*}}$.

${ }^{11}$ In case $G(0+)=\varnothing$, it can be assumed that $\tau \in\left(0, \varsigma_{j}\right]$, for all $j$, and, as said before, we then assume that for all $P$, $s_{i}=\tau \Rightarrow u_{i}=\bar{u}, \quad \bar{u}$ some arbitrarily given element in $U^{\prime}$ (in $\breve{U}(0) \cap U^{\prime}$ in case $U$ is replaced by $\tilde{U}(t)$, $u \in \breve{U}(0) \Rightarrow u \in \breve{U}\left(\tau_{n}\right)$ for $n$ large). 
$\left(p_{\infty}, \mu\right)=0$, a contradiction. Hence, $\left(p_{\infty}, \lim _{s \downarrow 0} \mu([s, \infty))\right) \neq 0$.

When $G(0+) \neq \varnothing$ and 0 is a right Lebesgue point of $f^{*}(t)$ (i.e. (10) ( $\beta$ ) holds), we can choose the sequence $\tau_{n}=0$. Evidently, by (29), $g_{x}^{j}\left(0, x^{*}(0)\right) f^{*}(0)+g_{t}^{j}\left(0, x^{*}(0)\right)=0$ when $j \in G(0+)$, so again $\hat{g}_{x}^{j}(0) v_{0}>0, \quad v_{0}=v^{*}-f^{*}(0)$, and we get the same conclusion regarding $\mu_{j}([0, s])$ (with $\left(p_{\infty}, \lim _{s \downarrow 0} \mu([s, \infty))\right) \neq 0$ again) and in this case, (as well as in the case that $(t, x) \rightarrow\left(f^{0}, f\right)$ is differentiable, i.e. (10) ( $\gamma$ ), see Appendix), we don't need the assumption that $G(0+)$ contains a single element ${ }^{12}$. In fact, when (8) holds, in Theorem 2, we can assume $\mu_{j}(0):=\lim _{s \downarrow 0} \mu_{j}([0, s])=0$.

Define $\mu_{j}(\infty)=\inf _{s \geq 0} \mu_{j}([s, \infty))$. Note that, by (23), $\mu_{j}\left(\left[T^{\prime \prime}, \infty\right)\right)=0$ for $T^{\prime \prime}$ large enough if $j \in G_{*}^{\infty}$. Assume that a $c^{*} \in C^{\infty}$ and positive numbers $\hat{T}$ and $\gamma_{*}$ exist such that

$$
g_{x}^{j}\left(t, x^{*}(t)\right) C(t, s) c^{*} \geq \gamma_{*}, j \in G^{*}:=G_{\infty} \backslash G_{*}^{\infty}, \text { when } \hat{T} \leq s \leq t
$$

(see the end of Theorem 2). For $s \geq \hat{T}$

$$
\begin{aligned}
p(s) c^{*} & =p_{\infty} C(\infty, s) c^{*}+\sum \int_{[s, \infty)} g_{x}^{j}\left(t, x^{*}(t)\right) C(t, s) c^{*} \mathrm{~d} \mu_{j}(t) \\
& \geq p_{\infty} C(\infty, s) c^{*}+\gamma_{*} \sum_{j \in G^{*}} \int_{[s, \infty)} \mathrm{d} \mu_{j}(t)
\end{aligned}
$$

Assume for the moment that $\liminf _{s \rightarrow \infty}|p(s)|=0$. Choose a sequence $s^{n} \rightarrow \infty$ such that $p\left(s^{n}\right) \rightarrow 0$. Letting $s=s^{n} \rightarrow \infty$ in the preceding inequality, and using $\pi C\left(\infty, s^{n}\right) \rightarrow \pi I$ (see Appendix, Lemma A), we get $0 \geq p_{\infty} c^{*}+\gamma_{*} \sum_{j \in G^{*}} \mu_{j}(\infty) \geq \gamma_{*} \sum_{j \in G^{*}} \mu_{j}(\infty)$, so $\sum \mu_{j}(\infty)=0$.

Can $\left(\liminf _{s \rightarrow \infty}|p(s)|, \lim _{s \rightarrow \infty} \sum_{j \in G^{*}} \mu_{j}([0, s])\right)=0$ ? No, we have shown that then $\sum_{j \in G^{*}} \mu_{j}(\infty)=0$, and then $0=\liminf _{s \rightarrow \infty}|p(s)|=\left|p_{\infty}\right|$, so $\left(p_{\infty}, \mu\right)=0$, a contradiction. So when (58) holds

$$
\left(\liminf _{s \rightarrow \infty}|p(s)|, \lim _{s \rightarrow \infty} \sum_{j \in G^{*}} \mu_{j}([0, s])\right) \neq 0
$$

Finally, assume that both (58) and (8) are satisfied. By contradiction assume now that $\left(\liminf _{s \rightarrow \infty}|p(s)|, \lim _{s \rightarrow \infty} \sum_{j \in G^{*}} \mu_{j}([1 / s, s])\right)=0 . \quad$ Then $\quad \sum_{j \in G^{*}} \mu_{j}(\infty)=0 \quad \Leftrightarrow \quad \lim _{s \rightarrow \infty} p(s)=p_{\infty} \quad$ and $\lim _{\hat{s} \rightarrow \infty} \sum_{j \in G^{*}} \mu_{j}([1 / s, \hat{s}])=\sum_{j \in G^{*}} \mu_{j}([1 / s, \infty))$, so $\left(p_{\infty}, \lim _{s \rightarrow \infty} \sum_{j \in G^{*}} \mu_{j}([1 / s, \infty))\right)=0$, a contradiction. So (58) and (8) imply

$$
\left(\liminf _{s \rightarrow \infty}|p(s)| \lim _{s \rightarrow \infty} \sum_{j \in G^{*}} \mu_{j}([1 / s, s])\right)=0
$$

\section{Proof of Theorem 1.}

We still keep the assumption that $a x(\infty)$ is maximized. Using lemmas in Appendix, note that (25)-(28) are implied by the basic smoothness assumptions, the growth conditions (11)-(13), (7), (14) (i.e. $g^{j}$ depends only on $\left.x^{i}, \quad i \leq n^{1}\right)$, and (15) implying that $z \rightarrow g^{j}\left(t, x^{*}(t)+z\right)$ is differentiable at 0 , uniformly in $t((27)$ follows from Lemma E in Appendix). Moreover, also (29), (30) evidently follow. So all the above results also hold in the situation of Theorem 1. Using the definition (57) the maximum condition (54) can be written

$$
\text { For a.e. } s, p(s)\left(f\left(s, x^{*}(s), u\right)-f^{*}(s)\right) \leq 0 \text { for all } u \in U
$$

Now,

${ }^{12}$ If (8) holds only for a subset $G^{\prime}$ of $G(0+)$ then it is easily seen that the collection $p_{\infty}, \mu_{j}, j \in G(0+) \backslash G^{\prime}$, $\lim _{s \downarrow 0} \mu_{j}([s, \infty)), \quad j \in G^{\prime} \cup[G(0+)$ is nonzero (at least one entity is nonzero). 
$\sum \int_{[s, \infty)} g_{x}^{j}\left(t, x^{*}(t)\right) C(t, s) \mathrm{d} \mu_{j}(t)=\sum \int_{[s, T} g_{x}^{j}\left(t, x^{*}(t)\right) C(t, s) \mathrm{d} \mu_{j}(t)+\sum \int_{[T, \infty)} g_{x}^{j}\left(t, x^{*}(t)\right) C(t, s) \mathrm{d} \mu_{j}(t) . \quad$ Using $\lim _{t \rightarrow \infty} \pi C(t, s)=\pi C(\infty, s)$, (5) and (14), $\sum \int_{[T, \infty)} g_{x}^{j}\left(t, x^{*}(t)\right) C(t, s) \mathrm{d} \mu_{j}(t) \rightarrow \sum g_{x}^{j}(\infty) \mu_{j}(\infty) C(\infty, s)$ when $T \rightarrow \infty$. Then, by (57), also $\lim _{T \rightarrow \infty} \sum \int_{[s, T)} g_{x}^{j}\left(t, x^{*}(t)\right) C(t, s) \mathrm{d} \mu_{j}(t)$ exists, and $p(s)=p_{\infty} C(\infty, s)+\lim _{T \rightarrow \infty} \sum \int_{[s, T)} g_{x}^{j}\left(t, x^{*}(t)\right) C(t, s) \mathrm{d} \mu_{j}(t)+\sum g_{x}^{j}(\infty) \mu_{j}(\infty) C(\infty, s)$. Hence, $p(s, T):=p_{\infty} C(T, s)+\sum \int_{[s, T)} g_{x}^{j}\left(t, x^{*}(t)\right) C(t, s) \mathrm{d} \mu_{j}(t)+\left(\sum g_{x}^{j}(\infty) \mu_{j}(\infty)\right) C(T, s) \rightarrow p(s)$ when $T \rightarrow \infty$.

Let $v_{j}(s)=\lim _{s^{\prime} \downarrow s} \mu_{j}\left(\left[0, s^{\prime}\right]\right)$. Let $J_{*}$ be the continuity points of all $s \rightarrow \mu_{j}([0, s])$. Write for the moment

$$
\hat{p}(s, T)=p_{\infty} C(T, s)+\sum \int_{[s, T)} g_{x}^{j}\left(t, x^{*}(t)\right) C(t, s) \mathrm{d} v_{j}(t)+\left(\sum g_{x}^{j}(\infty) \mu_{j}(\infty)\right) C(T, s)
$$

and $\hat{p}(s)=\lim _{T \rightarrow \infty} \hat{p}(s, T)$. Then ${ }^{13}$, for $s, T \in J_{*}, \quad \hat{p}(s, T)=p(s, T)$, and then $\hat{p}(s)=\lim _{T \rightarrow \infty} \hat{p}(s, T)=\lim _{T \rightarrow \infty, T \in J_{*}} p(s, T)=p(s), \quad s \in J_{*}$. With $\gamma^{j}:=\mu_{j}(\infty):=\inf _{s} \mu_{j}([s, \infty)) \quad\left(\gamma^{j} \quad\right.$ occurring in the definition of $\hat{p}_{\infty}$ ) it is well-known that $\hat{p}(s, T)$ satisfies $(16)^{14}$. Evidently, (61) yields

$$
\text { For a.e. } s, \hat{p}(s)\left(f\left(s, x^{*}(s), u\right)-f^{*}(s)\right) \leq 0 \text { for all } u \in U
$$

In Theorem 1, we have written $p(s, T)$ and $p(s)$ instead of $\hat{p}(s, T)$ and $\hat{p}(s)$.

Note that (6) implies that (58) holds, as $G^{\infty}=G_{*}^{\infty}, G(\infty)=G_{\infty} \backslash G_{*}^{\infty}$. Thus, (60) holds, which means that $\left(p_{\infty}+\hat{p}_{\infty}, \lim _{s \rightarrow \infty} \sum \mu_{j}([1 / s, s])\right) \neq 0$ in the situation of Theorem 1 , because then $p(\infty)$ exists and equals $p_{\infty}+\hat{p}_{\infty}$.

Proof of Remark 2. We give a proof for the case where $a x^{*}(\infty)$ is maximized. Note that $p(s) f\left(s, x^{*}(s), u^{*}(s)\right) \geq p(s) v$ for $v \in \operatorname{cof}\left(s, x^{*}(s), U\right)$ for $s \in J_{*}$, where $J_{*}$ is a set of Lebesgue points of $f^{*}():.=f\left(., x^{*}(),. u^{*}().\right)$ of full measure. The inequality holds for all $s>0$ in case of left continuity of $f^{*}$.

\section{Proof of a)}

Let $\Gamma_{t}:=\sum_{j} \gamma^{j} \hat{g}_{t}^{j}(\infty), \quad \Gamma^{\prime}:=\sum_{j \in G(\infty)} \gamma^{j}$ and recall $\hat{p}_{\infty}=\sum_{j \in G(\infty)} \gamma^{j} \hat{g}_{x}^{j}(\infty)$. Assume $p_{\infty}=0$. Assume by contradiction that $\Gamma^{\prime}>0$. Then, for all $s \in J_{*}$ large enough

$$
\begin{aligned}
&(\mathrm{d} / \mathrm{d} s) \sum_{j \in G(\infty)} \gamma^{j} g^{j}\left(s, x^{*}(s)\right) \\
&=\sum_{j \in G(\infty)} \gamma^{j} \hat{g}_{t}^{j}(s)+\sum_{j \in G(\infty)} \gamma^{j} \hat{g}_{x}^{j}(s) f\left(s, x^{*}(s), u^{*}(s)\right) \\
&=\Gamma_{t}+\left[\sum_{j \in G(\infty)} \gamma^{j} \hat{g}_{t}^{j}(s)-\Gamma_{t}\right]+\left[\sum_{j \in G(\infty)} \gamma^{j} \hat{g}_{x}^{j}(s)-\hat{p}_{\infty}\right] f\left(s, x^{*}(s), u^{*}(s)\right) \\
&+\left[\hat{p}_{\infty}-p(s)\right] f\left(s, x^{*}(s), u^{*}(s)\right)+p(s) f\left(s, x^{*}(s), u^{*}(s)\right) \\
& \geq \Gamma_{t}+\left[\sum_{j \in G(\infty)} \gamma^{j} \hat{g}_{t}^{j}(s)-\Gamma_{t}\right]+\left[\sum_{j \in G(\infty)} \gamma^{j} \hat{g}_{x}^{j}(s)-\hat{p}_{\infty}\right] f\left(s, x^{*}(s), u^{*}(s)\right) \\
&+\left[\hat{p}_{\infty}-p(s)\right] f\left(s, x^{*}(s), u^{*}(s)\right)+p(s) v_{s} \\
&=\Gamma_{t}+\left[\sum_{j \in G(\infty)} \gamma^{j} \hat{g}_{t}^{j}(s)-\Gamma_{t}\right]+\left[\sum_{j \in G(\infty)} \gamma^{j} \hat{g}_{x}^{j}(s)-\hat{p}_{\infty}\right] f\left(s, x^{*}(s), u^{*}(s)\right) \\
&+\left[\hat{p}_{\infty}-p(s)\right] f\left(s, x^{*}(s), u^{*}(s)\right)+\left[p(s)-\sum_{j \in G(\infty)} \gamma^{j} \hat{g}_{x}^{j}(\infty)\right] v_{s}+\hat{p}_{\infty} v_{s} \\
& \geq {\left[\sum_{j \in G(\infty)} \gamma^{j} \hat{g}_{t}^{j}(s)-\Gamma_{t}\right]+\left[\sum_{j \in G(\infty)} \gamma^{j} \hat{g}_{x}^{j}(s)-\hat{p}_{\infty}\right] f\left(s, x^{*}(s), u^{*}(s)\right) }
\end{aligned}
$$

\footnotetext{
${ }^{13}$ Observation A in Appendix may be consulted at this point.

${ }^{14}$ Compare e.g. Section 9.5 in [16].
} 


$$
\begin{aligned}
& \quad+\left[\hat{p}_{\infty}-p(s)\right] f\left(s, x^{*}(s), u^{*}(s)\right)+\left[p(s)-\hat{p}_{\infty}\right] v_{s}+\sum_{j \in G(\infty)} \gamma^{j}\left\{\hat{g}_{t}^{j}(\infty)+\hat{g}_{x}^{j}(\infty) v_{s}\right\} \\
& >0
\end{aligned}
$$

(the last term is $\geq \Gamma^{\prime} \varepsilon$, all square brackets $\rightarrow 0$ when $s \rightarrow \infty$ ). Then $\sum_{j \in G(\infty)} \gamma^{j} g^{j}\left(\infty, x^{*}(\infty)\right)>\sum_{j \in G(\infty)} \gamma^{j} g^{j}\left(s, x^{*}(s)\right) \geq 0$, which is a contradiction because $g^{j}\left(\infty, x^{*}(\infty)\right)=0$ for $j \in G(\infty)$. So $\Gamma^{\prime}$ cannot be $>0$ when $p_{\infty}=0$. Hence $\left(p_{\infty}, \sum_{j} \int_{(0, \infty)} \mathrm{d} v^{j}(t), \Gamma^{\prime}\right) \neq 0$ implies $\left(p_{\infty}, \sum_{j} \int_{(0, \infty)} \mathrm{d} v^{j}(t)\right) \neq 0$.

Proof of b) Assume by contradiction that $p_{\infty}=0$.

If $j \in G(\infty)$, then for some arbitrary large $s_{j} \in J_{*}$, we must have $(\mathrm{d} / \mathrm{d} s) g^{j}\left(s_{j}, x^{*}\left(s_{j}\right)\right)=g_{t}^{j}\left(s_{j}, x^{*}\left(s_{j}\right)\right)+g_{x}^{j}\left(s_{j}, x^{*}\left(s_{j}\right)\right) f\left(s_{j}, x^{*}\left(s_{j}\right), u^{*}\left(s_{j}\right)\right) \leq 0$. For $j^{*}>1$, for all $j$, for large $s_{j}$ the left hand side is $\approx \hat{g}_{t}^{j}(\infty)+\hat{g}_{x}^{j}(\infty) f\left(\infty, x^{*}(\infty), u^{*}(\infty)\right)$, i.e. it does not change much if $s_{j}$ is replaced by $s_{1}$. Hence, for $j \in G(\infty)$ (whether $j^{*}>1$ or $=1$ ), for large $s_{1}$, for $\varepsilon$ as in a),

$$
(\mathrm{d} / \mathrm{d} s) g^{j}\left(s_{1}, x^{*}\left(s_{1}\right)\right)=g_{t}^{j}\left(s_{1}, x^{*}\left(s_{1}\right)\right)+g_{x}^{j}\left(s_{1}, x^{*}\left(s_{1}\right)\right) f\left(s_{1}, x^{*}\left(s_{1}\right), u^{*}\left(s_{1}\right)\right) \leq \varepsilon / 4
$$

Using the vectors $v_{s}$ in a), for $s_{1}$ large we then have $\hat{g}_{x}^{j}\left(s_{1}\right)\left(v_{s}-f\left(s_{1}, x^{*}\left(s_{1}\right), u^{*}\left(s_{1}\right)\right)\right) \geq 3 \varepsilon / 4$. Because $\pi C(t, s) \approx \pi I$, when $t \geq s$, $s$ large, we finally get $\hat{g}_{x}^{j}(s) C(t, s)\left(v_{s}-f\left(s, x^{*}(s), u^{*}(s)\right)\right) \geq \varepsilon / 2$ when $s=s_{1}$ is large. When $s$ is large $j \notin G(\infty)$ means $j \notin G(t), \quad t \geq s$. Using $\gamma^{j}=0$ and (22) (which even holds for $\left.v(s)=v_{s}-f^{*}(s), \quad v_{s} \in \operatorname{cof}\left(s, x^{*}(s), U\right)\right)$, for $s \in J_{*}$ large, we get $0 \geq \sum_{j} \hat{g}_{x}^{j}(t) C(t, s) v(s) \mathrm{d} v^{j}(t)=\sum_{j \in G(\infty)} \int_{[s, \infty)} \hat{g}_{x}^{j}(t) C(t, s) v(s) \mathrm{d} v^{j}(t) \geq \sum_{j \in G(\infty)} \int_{[s, \infty)}(\varepsilon / 2) \mathrm{d} v^{j}(t)$. Hence, both for $j \in G(\infty)$ and for $j \notin G(\infty), \quad \int_{[s, \infty)} \mathrm{d} v^{j}(t)=0$ for some (large) $s$.

Next, let $s_{*}=\inf \left\{s: \int_{[s, \infty)} \mathrm{d} v^{j}(t)=0\right.$ for all $\left.j\right\}<\infty$. By contradiction, assume $s_{*}>0$. Let $v_{*}\left(s_{*}\right):=v_{s_{*}}:=\sum_{k} \lambda_{k} f\left(s_{*}, x^{*}\left(s_{*}\right), u^{k}\right) \in \operatorname{cof}\left(s_{*}, x^{*}\left(s_{*}\right), U\right)$, where $v_{s_{*}}$ has the property that $\hat{g}_{t}^{j}\left(s_{*}\right)+\hat{g}_{x}^{j}\left(s_{*}\right) v_{*}\left(s_{*}\right)=: \varepsilon^{\prime}>0$ for $j \in G\left(s_{*}\right)$, see b). By continuity, for any $s<s_{*}$ and close to $s_{*}$,

$$
\hat{g}_{t}^{j}(s)+\hat{g}_{x}^{j}(s) v_{*}(s) \geq 3 \varepsilon^{\prime} / 4 \text { for } j \in G\left(s_{*}\right) .
$$

There exist $s_{j} \in J_{*}, \quad s_{j}<s_{*}$ and arbitrarily close to $s_{*}$, such that for $j \in G\left(s_{*}\right)$, $\mathrm{d} g^{j}\left(s_{j}, x^{*}\left(s_{j}\right)\right) / \mathrm{d} s=\hat{g}_{t}^{j}\left(s_{j}\right)+\hat{g}_{x}^{j}\left(s_{j}\right) f^{*}\left(s_{j}\right) \leq 0$. If $j^{*}>1$, for all $j$, the left hand side is $\approx \hat{g}_{t}^{j}\left(s_{*}\right)+\hat{g}_{x}^{j}\left(s_{*}\right) f^{*}\left(s_{*}\right)$, so for $s_{1}$ close to $s_{*}, \quad \mathrm{~d} g^{j}\left(s_{1}, x^{*}\left(s_{1}\right)\right) / \mathrm{d} s=\hat{g}_{t}^{j}\left(s_{1}\right)+\hat{g}_{x}^{j}\left(s_{1}\right) f^{*}\left(s_{1}\right) \leq \varepsilon^{\prime} / 4$ for all $j \in G\left(s_{*}\right)$ whether $j^{*}>1$ or $j^{*}=1$. Combining this inequality with (63), we get for $s=s_{1} \in J_{*}, \quad s$ close to $s_{*}$ that $\hat{g}_{t}^{j}(s)+\hat{g}_{x}^{j}(s)\left(v_{*}(s)-f^{*}(s)\right) \geq \varepsilon^{\prime} / 2$ for $j \in G\left(s_{*}\right)^{15}$. From this, we finally get, by Lipschitz continuity of $t \rightarrow C(t, s)$ on $\left[0, s_{*}\right]$, uniformly in $s$, that there exists a $s=s^{*}<s_{*}, s^{*} \in J_{*}$ and close to $s_{*}$, such that $g_{x}^{j}\left(s^{*}, x^{*}\left(s^{*}\right)\right) C\left(t, s^{*}\right)\left(v_{*}\left(s^{*}\right)-f^{*}\left(s^{*}\right)\right) \geq \varepsilon^{\prime} / 4$ when $t \in\left[s^{*}, s_{*}\right]$, and $s^{*}$ can be chosen so close to $s_{*}$

\footnotetext{
${ }^{15}$ As an alternative to the left continuity assumption on $f^{*}\left(\right.$.) in Remark 2 (for $j^{*}>1$ ), we may assume that, if $\left(p_{0}, p_{\infty}\right)=0$ in the necessary conditions, then these conditions imply left continuity of $f^{*}($.$) on (0, \infty)$.
} 
that $g^{j}(t)>0$ when $t \in\left[s^{*}, s_{*}\right], \quad j \notin G\left(s^{*}\right)$. The last inequality and (22) then yields $0 \geq \sum_{j} \int_{\left[s^{*}, s_{*}\right.} \hat{g}_{x}^{j}(t) C\left(t, s^{*}\right)\left(v\left(s^{*}\right)-f^{*}\left(s^{*}\right)\right) \mathrm{d} v^{j}(t) \geq \sum_{j \in G\left(s_{*}\right)} \int_{\left[s^{*}, s_{*}\right.}\left(\varepsilon^{\prime} / 4\right) \mathrm{d} v^{j}$, which gives $\int_{\left[s^{*}, s_{*}\right]} \mathrm{d} v^{j}(t)=0$ both for $j \in G\left(s_{*}\right)$ and for $j \notin G\left(s_{*}\right)$ and so $\int_{\left[s^{*}, \infty\right)} \mathrm{d} v^{j}(t)=0$ for all $j$. Evidently we cannot have $s_{*}>0$, so $s_{*}=0$. Thus $p_{\infty}=0 \Rightarrow \sum_{j} \int_{(0, \infty)} \mathrm{d} v(t)=0$, contradicting $\left(p_{\infty}, \sum_{j} \int_{(0, \infty)} \mathrm{d} v(t)\right) \neq 0$. Hence $p_{\infty} \neq 0$.

Proof of Remark 4. We construct an auxiliary problem: assume for given functions $w_{k}(),. k=1, \cdots, k^{*}$, that we want to maximize $\operatorname{ax}(\infty)$ subject to

$$
\dot{x}=f\left(t, x,\left(1-\sum_{k} z_{k}\right) u+\sum_{k} z_{k} w_{k}(t)\right), \quad t \in[0, \infty)
$$

$w_{k}(t) \in U, \quad\left|w_{k}(.)-u^{*}(.)\right|_{\infty} \leq 1, \quad w_{k}($.$) measurable. Here z_{k}, \quad k=1, \cdots, k^{*}$, are auxiliary states, governed by $\dot{z}_{k}=\alpha_{k} 1_{[0,1)}(t), \quad \alpha_{k} \in[0,1], \quad z(0)=0$, where $\alpha_{k}$ are auxiliary controls. Write $\alpha=\left(\alpha_{1}, \cdots, \alpha_{k^{*}}\right) \in[0,1]^{k^{*}}$. For $\varepsilon>0$, define $\hat{A}(t):=\{(t, u): t \in[0,1 / \varepsilon), \quad u \in U \cap B(0,1 / \varepsilon)$, $g^{j}\left(t, x^{*}(t), u\right)>\varepsilon$ if $\left.t \in\left[a_{j}, b_{j}\right], \quad j>j^{*}\right\}, \breve{U}_{\varepsilon}(t):=\{u:(t, u) \in \hat{A}(t)\}$, and let $U_{\varepsilon}(t):=\left\{u^{*}(t)\right\} \cup \breve{U}_{\varepsilon}(t)$.

Below, $\varepsilon$ is so small that $\breve{U}_{\varepsilon}(0) \neq \varnothing$. Given any measurable control functions $\alpha(),. \breve{u}(t), \alpha(t) \in[0,1]^{k^{*}}$, $\breve{u}(t) \in U_{\varepsilon}(t)$, let $x^{\breve{u}(.), \alpha(.)}(t), \quad z_{k}^{\alpha(.)}($.$) be the solutions of (64) and \dot{z}_{k}=\alpha_{k}$ corresponding to $\breve{u}(),. \alpha($.$) .$ For any $\delta$, there exists a $\delta^{\prime} \in\left(0,1 / k^{*}\right]$ such that if meas $(\{t: \alpha(t) \neq 0\}) \leq \delta^{\prime}$ and meas $\left(\left\{t: \breve{u}(t) \neq u^{*}(t)\right\}\right) \leq \delta^{\prime}$, then $\left|\pi x^{\breve{u}(\cdot), \alpha(\cdot)}(t)-\pi x^{*}(t)\right| \leq \delta$ for all $t$, by Lemmas B and C in Appendix, hence, by continuity of $(x, u) \rightarrow g^{j}(t, x, u)$ at $\left(t, x^{*}(t), u^{*}(t)\right)$, uniformly in $t$, for some $\delta^{*} \in\left(0,1 / k^{*}\right]$, $g^{j}\left(t, x^{\breve{u}(\cdot), \alpha(\cdot)}(t),\left(1-\sum_{k} z_{k}\right) \breve{u}(t)+\sum z_{k} w_{k}(t)\right) \geq 0, \quad j>j^{*}$, for $t \in\left\{t: \breve{u}(t) \neq u^{*}(t)\right\} \cap\left[a_{j}, b_{j}\right]$, when meas $(\{t: \alpha(t) \neq 0\}) \leq \delta^{*}$ and meas $\left(\left\{t: \breve{u}(t) \neq u^{*}(t)\right\}\right) \leq \delta^{*}, \quad\left(\delta^{\prime}\right.$ and $\delta^{*}$ perhaps dependent on $\left.\varepsilon\right)$. In the auxiliary problem the constraints are the terminal constraints (4), meas $(\{t: \alpha(t) \neq 0\}) \leq \delta^{*}$, $\left(\left\{t: \breve{u}(t) \neq u^{*}(t)\right\}\right) \leq \delta^{*}, \quad g^{j}\left(t, x^{\breve{u}(.), \alpha(.)}(t)\right) \geq 0$ for all $t \in\left[a_{j}, b_{j}\right]$ for $j \leq j^{*}$,

$g^{j}\left(t, x^{\breve{u}(\cdot), \alpha(\cdot)}(t),\left(1-\sum_{k} z_{k}^{\alpha(\cdot)}(t)\right) u^{*}(t)+\sum z_{k}^{\alpha(\cdot)}(t) w_{k}(t)\right) \geq 0$ for all $t \in\left[a_{j}, b_{j}\right]$ for $j>j^{*}, \quad \breve{u}(t) \in U_{\varepsilon}(t)$.

Hence, if $(\breve{u}(),. \alpha()$.$) is admissible in the auxiliary problem, we have seen that$ $\tilde{u}(t):=\left(1-\sum_{k} z_{k}^{\alpha(\cdot)}(t)\right) \breve{u}(t)+\sum z_{k}^{\alpha(\cdot)}(t) w_{i}(t)$ is admissible in the original problem when meas $\left(\left\{t: \breve{u}(t) \neq u^{*}(t)\right\}\right) \leq \delta^{*}$, meas $(\{t: \alpha(t) \neq 0\}) \leq \delta^{*}$. We assume that $\delta^{*}$ is $\leq \tilde{\delta} / 2 k^{*}$ (for $\tilde{\delta}$ see the beginning of Remark 4, then $\left|\sum z_{k}^{\alpha(\cdot)}(t)\left(w_{i}(t)-u^{*}(t)\right)\right| \leq \tilde{\delta} / 2$ and then, for $\alpha^{*}(.) \equiv 0$, the property related to $X^{\delta}(t)$ in Remark 3 is satisfied in the auxiliary problem for $\left.\delta=\tilde{\delta} / 2 k^{*}\right)$. So, in the auxiliary problem, $\breve{u}^{*}(.) \equiv u^{*}(),. \quad \alpha^{*}(.) \equiv 0$ are optimal in the set of controls $\left\{(\breve{u}(t), \alpha(t)): \alpha(t) \in[0,1]^{k^{*}}\right.$, meas $(\{t: \alpha(t) \neq 0\}) \leq \delta^{*}, \quad$ meas $\left.\left(\left\{t: \breve{u}(t) \neq u^{*}(t)\right\}\right) \leq \delta^{*}, \quad \breve{u}(t) \in U_{\varepsilon}(t)\right\}$. The arguments in the proof of Theorem 2 apply also in the present situation, with one modification: For $j>j^{*}$, the inequality $g^{j} \geq 0$ in 
$\left[s_{i}^{*}, s_{i}^{*}+d \breve{c}_{i}\right]$ for $x=x\left(t, d c, P^{*}\right), \quad z_{k}=z_{k}\left(t, d c, P^{*}\right)$ automatically holds for $d \in\left(0, \delta^{*}\right]$. Hence the arguments in the section between (50) and (51) are not needed ${ }^{16}$ (and do not work) for $j>j^{*}$.

The necessary conditions in Theorem 2 are now applied to this auxiliary system (they apply even when admissible controls are restricted as above, see the inequalities involving $\delta^{*}$, and even for $U$ replaced by $[0,1]^{k^{*}} \times U_{\varepsilon}(t)$, see the end of Remark $3^{17}$. In the auxiliary system, the linearized system is $\dot{q}^{z}=0$, $\dot{q}^{x}=f_{u}^{*}(t) \hat{w}(t) q^{z}+f_{x}^{*} q^{x}$, where $\hat{w}(t)$ is the transposed of $\left(\hat{w}_{1}(r), \cdots, \hat{w}_{k}^{*}(r)\right):=\left(w_{1}()-.u^{*}(),. \cdots, w_{k}^{*}()-.u^{*}().\right)$. The resolvent of the linearized system becomes

$$
\left[\begin{array}{cc}
I & 0 \\
B(t, s) & C(t, s)
\end{array}\right], B(t, s)=\int_{s}^{t} C(t, r) f_{u}^{*}(r) \hat{w}(r) \mathrm{d} r
$$

where $(\mathrm{d} / \mathrm{d} t) B(t, s)=f_{u}^{*}(t) \hat{w}(t)+f_{x}^{*}(t) B(t, s), \quad B(s, s)=0$. From Lemma A in Appendix, we get that

$$
\left|B_{i}(t, s)\right| \leq Q \int_{s}^{t}|\hat{w}(s)| \mathrm{d} s,\left|\dot{B}_{i}(t, s)\right| \leq Q \mathrm{e}^{-\omega t} \int_{s}^{t}|\hat{w}(s)| \mathrm{d} s, i \leq n^{1}
$$

for some constant $Q$, independent of $t$ and $s \leq t$, where $B_{i}$ is the $i$ 'th row of $B$ and $\omega=(1 / 2) \min \left\{a, b-\left(n-n^{1}\right) \kappa\right\} \quad$ (to apply Lemma A, note that for $y=\left(x^{1}, \cdots, x^{n^{1}}\right), \quad z=\left(x^{n^{1}+1}, \cdots, x^{n}\right)$, in an obvious notation, $\quad \dot{q}^{y}=\gamma+f_{y}^{y^{*}} q^{y}+f_{z}^{y^{*}} q^{z}, \quad \dot{q}^{z}=\psi+f_{y}^{z^{*}} q^{y}+f_{z}^{z^{*}} q^{z}, \quad$ where $\quad \gamma=f_{u}^{y^{*}} \hat{w}, \quad \psi=f_{u}^{z^{*}} \hat{w}$, $\left.\left|f_{u}^{y^{*}}\right| \leq A \mathrm{e}^{-a t}, \quad\left|f_{u}^{z^{*}}\right| \leq C \mathrm{e}^{\kappa t}\right)$. Note that (22), or actually (54), applied to the auxiliary system, holds for $s=0$ for the limit point $(\alpha, 0)=\lim _{n} \breve{v}\left(\tau_{n}\right), \breve{v}\left(\tau_{n}\right)=\left(\alpha,(1 /(n+1))\left(f\left(\tau_{n}, x^{*}\left(\tau_{n}\right), \breve{u}\right)-f^{*}\left(\tau_{n}\right)\right)\right), \breve{u} \in U(0)$ given, $\alpha$ any given element in $[0,1]^{k^{*}}$ (see remarks subsequent to (55)). From this we get, for $s=0$ and $\alpha=\left(0, \cdots, 0, \alpha_{k}, 0, \cdots, 0\right), \quad \alpha_{k} \in[0,1]$ and $w()=.\hat{w}_{k}($.$) that$

$$
0 \geq \alpha_{k}\left\{p_{\infty} \int_{0}^{\infty} C(\infty, r) f_{u}^{*}(r) w(r) \mathrm{d} r+\int_{[0, \infty)} \sum_{j=1}^{j^{* *}}\left[\hat{g}_{x}^{j}(t) \int_{0}^{t} C(t, r) f_{u}^{*}(r) w(r) \mathrm{d} r+\hat{g}_{u}^{j}(t) w(t)\right] \mathrm{d} \mu_{j}(t)\right\}
$$

From now on assume $\alpha_{k}=1$ in (67). Moreover, for $\alpha=0, \quad u \in \breve{U}_{\varepsilon}(s)$, from (54) applied to the auxiliary system, we get that (54) holds as written. Finally, $\left(p_{\infty}, \mu\right) \in A, A$ defined below.

Let $A$ consist of all pairs $\left(p_{\infty}, \mu\right)$ such that $p_{\infty}$ satisfies (52) and $\mu:=\left(\mu_{1}, \cdots, \mu_{j^{* *}}\right)$ satisfies $\mu_{j} \geq 0$, $\mu_{j}\left(\left[J^{*}(j, \beta)\right)=0\right.$ for all $\beta>0$, and (56) for $j \leq j^{*}$, with $\left|p_{\infty}\right|+\sum \mu_{j}([0, \infty))=1$. Let $A_{w}^{\varepsilon} \subset A$, $w=w(.) \in W^{*}, \quad \varepsilon>0$, consist of all pairs $\left(p_{\infty}, \mu\right) \in A$ such that (67) holds for the given $w$ and (54) are satisfied for all $u \in \breve{U}_{\varepsilon}(s)$, for a.e. $s$, in particular for $s>0, \quad u \in \breve{U}_{\varepsilon}(s) \cap U^{\prime}, s \in J_{u}$, and $s=0, \quad v_{0}$ any given cluster point of any given sequence $v\left(\tau_{n}\right)$, each $v\left(\tau_{n}\right)$ corresponding to some collection $u_{i}^{\tau_{n}}$ from $U_{\varepsilon}\left(\tau_{n}\right)$.

Let $\Gamma$ consist of all finite set $\Gamma^{\prime}=\left\{w_{1}, \cdots, w_{k^{*}}, \varepsilon_{1}, \cdots, \varepsilon_{k^{*}}\right\}, \quad w_{k} \in W^{*}, \quad \varepsilon_{k}>0$. We have just proved that for each $\Gamma^{\prime}, A_{\Gamma^{\prime}}:=\bigcap_{k} A_{w_{k}}^{\varepsilon_{k}}$ is nonempty, so by compactness $\bigcap_{\Gamma^{\prime} \in \Gamma} A_{\Gamma^{\prime}}$ is nonempty (the weak* topology is applied on the $\mu^{\prime}$ s ). Let $a^{*}:=\left(p_{\infty}, \mu^{*}\right) \in A$ be any given element in the latter intersection. Then, for $a^{*}$, both

\footnotetext{
${ }^{16}$ Thus we don't need (and often don't have!) differentiability of $t \rightarrow g^{j}\left(t, x,\left(1-\sum_{k} z_{k}\right) u^{*}(t)+\sum_{k} z_{k} \omega_{k}(t)\right)$ for $j>j^{*}$.

${ }^{17}$ The growth conditions related to $\left(z^{1}, \cdots, z^{k^{k}}, x^{1}, \cdots, x^{n^{1}}\right)$ are of the same type as those related to $x^{1}, \cdots, x^{n^{1}}$ in problem (1)-(4). Note that the perturbations $u_{i}$ now belong to $\breve{U}_{s}(t)$.
} 
(67) holds for all $w(.) \in W^{*}$, and (54) is satisfied for $u \in U(s)$ for a.e. s. (To obtain this last property, preferably the set $J^{*}$ of point $s$ for which (54) holds should be independent of the $\left(p_{\infty}, \mu\right)$ 's in each $A_{\Gamma^{\prime}}$, one can use that (54) now holds for $a^{*}$, for $u \in U(s) \cap U^{\prime}$ for $s>0, s \in J_{u}$ and hence by earlier limit arguments (54) holds for $a^{*}$, for all $u \in U(t)$, for a.e. $s$ ). We also have that (54) is satisfied by $a^{*}$ for $s=0$ for any given cluster point $v_{0}$ for any given sequence $v\left(\tau_{n}\right)$, each $v\left(\tau_{n}\right)$ corresponding to some $\left\{u_{i}^{\tau_{n}}\right\}_{i, n} \subset U_{\varepsilon}(t)$ for some $\varepsilon>0$.

The proof of $\left(p_{\infty}, \lim _{s \downarrow 0} \hat{\mu}([s, \infty)), \breve{\mu}\right) \neq 0$, is the same as the proof for the analogous condition in the case $j^{*}=j^{* *}$, noting that $v^{*}=\sum \lambda_{i}^{*} f\left(0, x_{0}, u_{i}^{*}\right)$ for some $u_{i}^{*} \in U(0)$ means for some $\varepsilon>0$, $v_{*}(t)=\sum \lambda_{i}^{*} f\left(t, x^{*}(t), u_{i}^{*}\right) \in \operatorname{cof}\left(t, x^{*}(t), U_{\varepsilon}(t)\right)$ for $t$ near 0 (so $\left(p_{\infty}, \lim _{s \downarrow 0} \hat{\mu}([s, \infty)), \breve{\mu}\right)=0$, $\sum_{j \in G(0+)} \mu_{j}(0)>0$ again leads to a contradiction in the same way as before $)^{18}$. Similarly, $\left(\liminf _{s \rightarrow \infty}|p(s)|, \lim _{s \rightarrow \infty} \hat{\mu}([0, s)), \breve{\mu}\right) \neq 0$ has essentially the same proof as before. To show $\breve{\mu} \in L_{\infty}\left([0, \infty), \mathbb{R}^{j^{* *}-j^{*}}\right)$ in case $\left[\hat{g}_{u}^{j}(t) \tilde{w}(t)-\delta\right] 1_{\left[a_{j}, b_{j}\right] \cap J^{*}(j, \beta)} \geq 0$ holds, we now assume $k^{*}=1$, and we replace $w()=.\hat{w}_{k}($.$) by \tilde{w} \phi$ in the definition of $B(t, s)$, where $\phi \in L_{\infty}([0, \infty), \mathbb{R}), \phi \geq 0$. Then from (66), we get

$$
\left|B_{i}(t, 0)\right| \leq K^{\prime} \int_{0}^{\infty} \phi(s) \mathrm{d} s \text { for some constant } K^{\prime}, i \leq n^{1}
$$

Using the inequality

$$
0 \geq\left\{p_{\infty} B(\infty, 0)+\int_{[0, \infty)} \sum_{j=1}^{j^{* *}}\left[\hat{g}_{x}^{j}(t) B(t, 0)+\hat{g}_{u}^{j}(t) w(t)\right] \mathrm{d} \mu_{j}(t)\right\}
$$

(i.e. (67)), we get

$$
\begin{aligned}
& \sum_{i \leq n^{*}}-p_{\infty}^{i} B_{i}(\infty, 0)-\int_{[0, \infty)} \sum_{j=1}^{j^{* *}} \hat{g}_{x}^{j}(t) B(t, 0) \mathrm{d} \mu_{j}(t) \\
& \geq \int_{[0, \infty)} \sum_{j>j^{*}} \hat{g}_{u}^{j}(t) \tilde{w}(t) \phi(t) \mathrm{d} \mu_{j}(t) \geq \sum_{j>j^{*}} \int_{[0, \infty)} \delta \varphi(t) \mathrm{d} \mu_{j}(t)
\end{aligned}
$$

Note that $\hat{g}_{x}^{j}(t) B(t, 0)=\sum_{i \leq n} \hat{g}_{x^{i}}^{j}(t) B_{i}(t, 0)$. So, from (68), (70) and $\sum \mu_{j}([0, \infty)) \leq 1, \quad \sum_{i}\left|p_{\infty}^{i}\right| \leq n$, it follows that $n K^{\prime} \int_{0}^{\infty} \phi(t) \mathrm{d} t+\left[j^{* *} n^{1} \sup _{t, j}\left|\hat{g}_{x}^{j}(t)\right|\right] K^{\prime} \int_{0}^{\infty} \phi(t) \mathrm{d} t \geq \sum_{j>j} \int_{[0, \infty)} \delta \phi(t) \mathrm{d} \mu_{j}(t)$. Then $\sup _{\psi \in L_{\infty}([0, \infty), \mathbb{R}),|\psi|_{1} \leq 1}\left|\int_{[0, \infty)} \psi(t) \mathrm{d} \mu_{j}(t)\right|<\infty, \quad j>j^{*}$ (note the $L_{1}$-norm on $\psi$ ). But then $\mu_{j}, j>j^{*}$, can be represented by nonnegative functions $\mu^{j}$ in $L_{\infty}([0, \infty), \mathbb{R})$, in fact in $L_{\infty}([0, \infty), \mathbb{R}) \cap L_{1}([0, \infty), \mathbb{R})$, because $\mu_{j}$ is bounded.

Let us finally show that $\left(p_{\infty}, \lim _{s \downarrow} \hat{\mu}([s, \infty))\right)=0 \Rightarrow \breve{\mu}=0$ when $\tilde{w}($.$) exists. By (66), for \hat{w}=\tilde{w}$, $\left|\mathrm{d} B_{i}(t, s) / \mathrm{d} t\right| \leq Q(t-s)|\tilde{w}(.)|_{\infty} \mathrm{e}^{-\omega t}, \quad Q$ independent of $t$ and $s \in[0, t], \quad i \leq n^{1}$. So, for $t>T$, $\left(Q|\tilde{w}(.)|_{\infty} / \omega^{2}\right) \mathrm{e}^{-\omega T} \geq\left|B_{i}(t, T)\right|=\left|\int_{T}^{t} C_{i}(t, r) f_{u}^{*}(r) \tilde{w}(r) \mathrm{d} r\right|, \quad i \leq n^{1}$. By this inequality, there exists a $T^{\prime}$ such that, for $j>j^{*}, \quad \sum_{i \leq n} \hat{g}_{x^{i}}^{j}(t) \int_{T^{\prime}}^{t} C_{i}(t, r) f_{u}^{*}(r) \tilde{w}(r) \mathrm{d} r+\hat{g}_{u}^{j}(t) \tilde{w}(t) \geq \delta / 2$ for $t \geq T^{\prime}, \quad t \in\left[a_{j}, b_{j}\right] \cap J^{*}(j, \beta)$. Next, for some $\xi$, for $t \in\left[0, T^{\prime}\right], \quad j>j^{*}, \quad \rho>0, \quad \hat{g}_{x}^{j}(t) \int_{\rho}^{t} C(t, r) f_{u}^{*}(r) \tilde{w}(r) \mathrm{e}^{\xi r} \mathrm{~d} r+\hat{g}_{u}^{j}(t) \tilde{w}(t) \mathrm{e}^{\xi t} \geq \delta \mathrm{e}^{\xi t} / 2$

\footnotetext{
${ }^{18}$ We can again let $\mu_{j}(0)=0, \quad j \leq j^{*}$ also in the present case, (67) does not change for this change of $u_{j}$.
} 
for $t \leq T^{\prime}$. To see this, for $k$ chosen such that $k \geq\left|\hat{g}_{x}(t)\right|, \quad n^{1} \max _{i \leq n^{1}}\left|C_{i}(t, r)\right|,\left|f_{u}^{*}(r)\right|,|\tilde{w}(r)|$ for $t \in\left[0, T^{\prime}\right]$, note that $\int_{0}^{t} k^{4} \mathrm{e}^{\xi r} \mathrm{~d} r \leq k^{4} \mathrm{e}^{\xi t} / \xi=\delta \mathrm{e}^{\xi t} / 2$ when $\xi=2 k^{4} / \delta$. By (67) and the two inequalities involving $\delta$, for

$$
\begin{aligned}
& w=\tilde{w}\left(\mathrm{e}^{\xi\left(t-T^{\prime}\right)} 1_{\left[\rho, T^{\prime}\right)}(t)+1_{\left[T^{\prime}, \infty\right)}(t)\right) \in W^{*} \text {, and for }\left(p_{\infty}, \lim _{s \downarrow 0} \hat{\mu}([s, \infty))\right)=0 \text {, for any } \rho>0 \\
& 0=-p_{\infty} \int_{\rho}^{\infty} C(\infty, r) f_{u}^{*}(r) w(r) \mathrm{d} r-\sum_{j \leq j^{*}} \int_{[\rho, \infty)} \hat{g}_{x}^{j}(t) \int_{\rho}^{t} C(t, r) f_{u}^{*}(r) w(r) \mathrm{d} r \mathrm{~d} \mu_{j}(t) \\
& \geq \sum_{j>j^{*}} \int_{[\rho, \infty)}\left[\hat{g}_{x}^{j}(t) \int_{\rho}^{t} C(t, r) f_{u}^{*}(r) w(r) \mathrm{d} r+\sum_{j>j^{*}} \hat{g}_{u}^{j}(t) w(t)\right] \mathrm{d} \mu_{j}(t) \\
& \geq \sum_{j>j^{*}} \int_{\rho}^{\infty}\left(\delta \mathrm{e}^{-\xi T^{\prime}} / 2\right) \mu^{j}(r) \mathrm{d} r
\end{aligned}
$$

But then $\mu^{j}=0, \quad j>j^{*}$, because $\rho$ was arbitrary. A contradiction of $\left(p_{\infty}, \lim _{s \rightarrow 0} \hat{\mu}([s, \infty)), \breve{\mu}\right) \neq 0$ has arisen, so $\left(p_{\infty}, \lim _{s \downarrow 0} \hat{\mu}([s, \infty))\right) \neq 0$.

\section{Conclusion}

The paper establishes necessary conditions for optimality in a smooth infinite horizon optimal control problem with unilateral state constraints and terminal constraints at the infinite horizon. The necessary conditions include a complete set of transversality conditions at infinity. The specific growth conditions placed upon the system in this paper can easily be modified, but strong growth conditions are in any case needed for the full set of necessary conditions to hold.

\section{Acknowledgements}

I was deeply grateful to the referees. Their detailed comments made it possible to correct omissions and improve the exposition.

\section{References}

[1] Halkin, H. (1974) Necessary Conditions for Optimal Control Problems with Infinite Horizons. Econometrica, 42, 267272. http://dx.doi.org/10.2307/1911976

[2] Michel, P. (1982) On the Transversality Condition in Infinite Horizon Problems. Econometrica, 50, 975-985. http://dx.doi.org/10.2307/1912772

[3] Seierstad, A. and Sydsaeter, K. (2009) Conditions Implying the Vanishing of the Hamiltonian at Infinity in Optimal Control Problems. Optimization Letters, 3, 507-512. http://dx.doi.org/10.1007/s11590-009-0128-7

[4] Aseev, S.M. and Veliov, V.M. (2011) Maximum Principles for Infinite-Horizon Optimal Control Problems with Dominating Discount. Research Report 2011-06 June, Operations Research and Control Systems, Institute of Mathematical Methods in Economics, Vienna University of Technology, Vienna.

[5] Benveneniste, L. and Scheinkman, J. (1982) Duality Theory for Dynamic Optimization Models in Economics. Journal of Economic Theory, 27, 1-19. http://dx.doi.org/10.1016/0022-0531(82)90012-6

[6] Seierstad, A. and K. Sydsaeter (1987) Optimal Control Theory with Economic Applications. Amsterdam, The Netherland.

[7] Seierstad, A. (1999) Necessary Conditions for Non-Smooth Infinite Horizon Control Problems. Journal of Optimization Theory and Applications, 103, 201-229. http://dx.doi.org/10.1023/A:1021733719020

[8] Pereira, F.L. and Silva, G.N. (2011) A Maximum Principle for Constrained Infinite Horizon Dynamic Control Systems. Preprints of the 18th IFAC World-Congress, Milano, 28 August-2 September 2011, 10207-10212.

[9] de Oliveira, V.A. and Silva, G.N. (2009) Optimality Conditions for Infinite Horizon Control problems with State Constraints. Nonlinear Analysis, 71, e1788-e1795.

[10] Aseev, S.M. and Veliov, V.M. (2012) Needle Variations in Infinite-Horizon Optimal Control. Research Report 2012-4, September, Operations Research and Control Systems, Institute of Mathematical Methods in Economics, Vienna University of Technology, Vienna.

[11] Aseev, S.M. and Kryazhimskii, A.V. (2004) The Pontryagin Maximum Principle and Transversality Conditions for a 
Class of Optimal Control Problems with Infinite Time Horizons. SIAM Journal on Control and Optimization, 43, 1094-1119.

[12] Weber, T.A. (2006) An Infinite-Horizon Maximum Principle with Bounds on the Adjoint Variable. Journal of Economic Dynamics and Control, 30, 229-241. http://dx.doi.org/10.1016/j.jedc.2004.11.006

[13] Arutyunov, A.V. and Aseev, S.M. (1977) Investigation of the Degeneracy Phenomenon of the Maximum Principle for Optimal Control with State Constraints. SIAM Journal on Control and Optimization, 35, 930-952. http://dx.doi.org/10.1137/S036301299426996X

[14] Vinter, R.B. and Ferreira, M.M.A. (1994) When Is the Maximum Principle for State Constrained Problems Nondegenerate? Journal of Mathematical Analysis and Applications, 187, 438-467. http://dx.doi.org/10.1006/jmaa.1994.1366

[15] Ferreira, M.M.A. and Fontes, F.A.C.C. (2004) Nondegeneracy and Normality in Necessary Conditions for Optimality: An Overview. Proceedings of the 6th Portuguese Conference on Automatic Control, CONTROLO, Faro, Portugal, 1-9 June 2004.

[16] Vinter, R.B. (2000) Optimal Control. Birkhäuser, Boston.

[17] Arutyunov, A.V., Karamzin, D.Y. and Pereira, F.L. (2011) The Maximum Principle for Optimal Control Problems with State Constraints by R.V. Gamkrelidze: Revisited. Journal of Optimization Theory and Applications, 149, 474493. http://dx.doi.org/10.1007/s10957-011-9807-5

[18] Arutyunov, A.V., Aseev, S.M. and Blagodatskikh, V.I. (1994) First Order Necessary Conditions in the Problem of Optimal Control of a Differential Inclusion with Phase Constraints. Russian Academy of Sciences Sbornik Mathematics, 79, 117-139. http://dx.doi.org/10.1070/sm1994v079n01abeh003493

[19] Arutyunov, A.V. (2000) Optimality Conditions: Abnormal and Degenerate Problems. Kluwer Academic, Dortdrecht. http://dx.doi.org/10.1007/978-94-015-9438-7

[20] Arutyunov, A.V. and Aseev, S.M. (1995) State Constraints in Optimal Control: The Degeneracy Phenomenon. Systems \& Control Letters, 26, 267-273. http://dx.doi.org/10.1016/0167-6911(95)00021-Z

[21] Rampazzo, F. and Vinter, R.B. (1999) A Theorem on the Existence of a Neighbouring Feasible Trajectory Satisfying a State Constraint, with Application to Optimal Control. IMA Journal of Mathematical Control and Information, 16, 335-351. http://dx.doi.org/10.1093/imamci/16.4.335

[22] Rampazzo, F. and Vinter, R.B. (2000) Degenerate Optimal Control Problems with State Constraints. SIAM Journal on Control and Optimization, 39, 989-1007. http://dx.doi.org/10.1137/S0363012998340223

[23] Bettiol, P. and Frankowska, H. (2007) Normality of the Maximum princIple for Nonconvex Constrained Bolza Problems. Journal of Differential Equations, 243, 2565-2569. http://dx.doi.org/10.1016/j.jde.2007.05.005

[24] Fontes, F.A.C.C. (2000) Normality in the Necessary Conditions of Optimality for Control Problems with State Constraints. Proceedings of the IASTED Conference on Control and Applications, Cancun, Mexico. 


\section{Appendix}

Below, for any matrix $A, \quad|A|=\sup _{|x| \leq 1}|A x|$.

Lemma A. Let $y=\left(x^{1}, \cdots, x^{n^{1}}\right), z=\left(x^{n^{1}+1}, \cdots, x^{n}\right), \quad x=(y, z), \quad n^{1}<n$, let

$$
F(t):=\left[\begin{array}{ll}
F_{y}^{y}(t) & F_{z}^{y}(t) \\
F_{y}^{z}(t) & F_{z}^{z}(t)
\end{array}\right], \quad G(t)=\left[\begin{array}{l}
\gamma(t) \\
\psi(t)
\end{array}\right]
$$

where, in $F(t)$, the matrices in the first row are respectively $n^{1} \times n^{1}$ and $n^{1} \times\left(n-n^{1}\right)$ and in the second row $\left(n^{1}-n\right) \times n^{1}$ and $\left(n^{1}-n\right) \times\left(n^{1}-n\right)$, and, in $G(t), \gamma(t)$ is $n^{1} \times 1$ and $\psi(t)$ is $\left(n-n^{1}\right) \times 1$, all entries being measurable functions of $t \in[0, \infty), \quad \gamma$ and $\psi$ locally integrable. Assume that for some positive numbers $A^{*}, B^{*}, C^{*}, a, b, \kappa, \quad\left|F_{y}^{y}(t)\right| \leq A^{*} \mathrm{e}^{-a t}, \quad\left|F_{z}^{y}(t)\right| \leq B^{*} \mathrm{e}^{-b t}, \quad\left|F_{y}^{z}(t)\right| \leq C^{*} \mathrm{e}^{\kappa t}, \quad\left|F_{z}^{z}(t)\right| \leq \kappa, \kappa<b$. Write $\left(q^{y}(t), q^{z}(t)\right)=: q(t) \in \mathbb{R}^{n^{1}} \times \mathbb{R}^{n-n^{1}}$ for the solution $q(t)$ on $[\tau, \infty), \tau \geq 0$, of $\dot{q}=F(t) q+G(t)$ for $q(\tau) \in \mathbb{R}^{n}$ given. Define $\omega=(1 / 2) \min \{a, b-\kappa\}$. Then for some positive number $Q$, only dependent on $A^{*}, B^{*}, C^{*}, a, b, \kappa, \quad\left|q^{y}(t)\right| \leq Q \cdot\left[|q(\tau)|+\int_{\tau}^{t}|\gamma(\rho)| \mathrm{d} \rho+\int_{\tau}^{t}|\psi(\rho)| \mathrm{e}^{-\kappa \rho} \mathrm{d} \rho\right]$, $\left|q^{z}(t)\right| \leq Q \cdot \mathrm{e}^{(\kappa+\omega) t}\left[|q(\tau)|+\int_{\tau}^{t}|\gamma(\rho)| \mathrm{d} \rho+\int_{\tau}^{t}|\psi(\rho)| \mathrm{e}^{-\kappa \rho} \mathrm{d} \rho\right]$. Hence $\left|\dot{q}^{y}(t)\right| \leq\left(A^{*}+B^{*}\right) Q \mathrm{e}^{-\omega t}\left[|q(\tau)|+\int_{\tau}^{t}|\gamma(\rho)| \mathrm{d} \rho+\int_{\tau}^{t}|\psi(\rho)| \mathrm{e}^{-\kappa \rho} \mathrm{d} \rho\right]$.

Lemma B. Let $f^{y}(t, y, z):[0, \infty) \times \mathbb{R}^{n_{*}} \times \mathbb{R}^{m^{*}} \rightarrow \mathbb{R}^{n_{*}}$ and $f^{z}(t, y, z):[0, \infty) \times \mathbb{R}^{n_{*}} \times \mathbb{R}^{m^{*}} \rightarrow \mathbb{R}^{m^{*}}$ be measurable in $t$, and with $y \rightarrow f^{y}(t, y, z), \quad z \rightarrow f^{y}(t, y, z), \quad y \rightarrow f^{z}(t, y, z)$ and $z \rightarrow f^{z}(t, y, z)$ Lipschitz continuous with Lipschitz constants $A^{*} \mathrm{e}^{-a t}, B^{*} \mathrm{e}^{-b t}, C^{*} \mathrm{e}^{\kappa t}$ and $\kappa$, respectively, $\kappa<b$. Write $x=(y, z)$, $\omega=(1 / 2) \min \{a, b-\kappa\}$. There exists a positive number $\Gamma$ such that the following properties hold: let $x(t)=(y(t), z(t))$ and $x_{*}(t)=\left(y_{*}(t), z_{*}(t)\right)$ be two solution on $[0, \infty)$ of $\dot{x}=f(t, x)=\left(f^{y}(t, x), f^{z}(t, x)\right)$, for $x(0)$, respectively $x_{*}(0)$ given, assumed to exist. Then for all $t$, $\left|y(t)-y_{*}(t)\right| \leq \Gamma\left|x(0)-x^{*}(0)\right|$ and $\left|z(t)-z_{*}(t)\right| \leq \Gamma\left|x(0)-x^{*}(0)\right| \mathrm{e}^{(\kappa+\omega) t}$, so $\left|\dot{y}(t)-\dot{y}_{*}(t)\right| \leq\left[\left(A^{*}+B^{*}\right) \Gamma \mathrm{e}^{-\omega t}\right]\left|x(0)-x^{*}(0)\right|$, which implies that $y(\infty)$ exists if $y_{*}(\infty)$ exists.

Note that $x($.$) and x_{*}($.$) do exist whenever f(t, x(0))$ and $f\left(t, x_{*}(0)\right)$ are integrable.

The proofs of the lemmas A and B are of a standard type and omitted in order to save space.

Let $T>0$ be given and let $J_{T}=[0, T]$. Let $\sigma\left(f, f^{*}\right):=\operatorname{meas}\left\{t: f(t, x) \neq f^{*}(t, x)\right.$ for at least one $\left.x\right\}$.

Lemma C. Let $F$ be a family of functions $f(t, x): J_{T} \times \mathbb{R}^{n} \rightarrow \mathbb{R}^{n}$, such that all $f(t, x)$ are Lipschitz continuous in $x$ with a common Lipschitz rank $\beta(t), \quad \beta$ integrable, and with $t \rightarrow f(t, x)$ measurable. Let $x^{*}($.$) be a solution of \dot{x}=f^{*}, \quad x^{*}(0)$ and $f^{*} \in F$ given (assumed to exist). Assume that all $f\left(t, x^{*}(t)\right)-f^{*}\left(t, x^{*}(t)\right), \quad f \in F$ are bounded by a common constant $\alpha$. Then a constant $\Gamma^{*}$ exists such that for any $f_{*} \in F$, for any given $x_{*}^{0}$, a solution of $\dot{x}_{*}=f_{*}, \quad x_{*}(0)=x_{*}^{0}$ exists, and $\left|x^{*}(t)-x_{*}(t)\right| \leq \Gamma^{*}\left[\left|x^{*}(0)-x_{*}(0)\right|+\sigma\left(f_{*}, f^{*}\right)\right]$ for all $t \in J_{T}$.

Proof of Lemma C. Note that $\int_{0}^{t}\left|f_{*}\left(s, x^{*}(s)\right)-f^{*}\left(s, x^{*}(s)\right)\right| \mathrm{d} s \leq 2 \alpha \sigma\left(f_{*}, f^{*}\right)$, so by Gronwall's inequality, $\left|x_{*}(t)-x^{*}(t)\right| \leq\left[\left|x^{*}(0)-x_{*}(0)\right|+2 \alpha \sigma\left(f_{*}, f^{*}\right)\right] \mathrm{e}^{\mathrm{j}_{0}^{t} \beta(s) \mathrm{d} s}$. 
Lemma D. Let $F$ be a family of functions $f(t, x): J_{T} \times \mathbb{R}^{n} \rightarrow \mathbb{R}^{n}$, all Lipschitz continuous in $x$ with a common Lipschitz rank $\beta(t), \beta$ integrable, and with $t \rightarrow f(t, x)$ measurable. Let $f^{*}$ be a given function in $F$ and let $x^{*}($.$) be a solution of \dot{x}=f^{*}, x^{*}(0)$ given (assumed to exist). We assume that all $f\left(t, x^{*}(t)\right)-f^{*}\left(t, x^{*}(t)\right), \quad f \in F$, are bounded by a common constant $\alpha$. We also assume that $x \rightarrow f^{*}(t, x)$ is differentiable at $\left(t, x^{*}(t)\right)$ for a.e. $t$. For $\left(f, q_{0}\right) \in F \times \mathbb{R}^{n}$, let $q()=.q^{f, q_{0}}($.$) be a solution$ of $\dot{q}=f_{x}^{*}\left(t, x^{*}(t)\right) q+f\left(t, x^{*}(t)\right)-f^{*}\left(t, x^{*}(t)\right), \quad q(0)=q_{0}, \quad q_{0}$ given. Then, for some $K^{*}>0$, for all $t$, $\left|q^{f, q_{0}}(t)\right| \leq K^{*}|d|$, when $\sigma\left(f, f^{*}\right) \leq d, \quad\left|q_{0}\right| \leq d$. Moreover, for some $K_{*}$ and some second order term $o_{*}(d)$, for all $t$, all $x^{f}(0)$, all $q_{0} \in B(0, d)$, all $f$ such that $\sigma\left(f, f^{*}\right) \leq d$, $\left|x^{f}(t)-x^{*}(t)-q^{f, q_{0}}(t)\right| \leq\left|x^{f}(0)-x^{*}(0)-q_{0}\right| K_{*}+o_{*}(d)$, where $x^{f}($.$) is the solution of \dot{x}=f, \quad x^{f}(0)$ given (it does exist).

Proof of Lemma D. The proof of $\left|q^{f, q_{0}}(t)\right| \leq K^{*} d$ follows from Lemma C. Let $z(t)=q^{f, q_{0}}(t)+x^{*}(t)$ and let $f=f^{*}$ on $C M \times \mathbb{R}^{n}$, meas $(M) \leq d$. We have that $\left|f^{*}(t, z(t))-f^{*}\left(t, x^{*}(t)\right)-f_{x}^{*}\left(t, x^{*}(t)\right) q^{f, q_{0}}(t)\right| \leq 2 \beta(t)\left|q^{f, q_{0}}(t)\right| \leq 2 \beta(t) K^{*} d$, and, by differentiability of $x \rightarrow f$ at $\left(t, x^{*}(t)\right)$ we have that $\left|f^{*}(t, z(t))-f^{*}\left(t, x^{*}(t)\right)-f_{x}^{*}\left(t, x^{*}(t)\right) q^{f, q_{0}}(t)\right| \leq o_{t}^{*}\left(\left|q^{f, q_{0}}(t)\right|\right) \leq o_{t}^{*}\left(d K^{*}\right)$ for some second order increasing term $r \rightarrow o_{t}(r) \leq 2 \beta(t) r$, so for

$$
\begin{aligned}
& \gamma(t):=f(t, z(t))-\dot{x}^{*}-\dot{q}^{f, q_{0}}=f(t, z(t))-f^{*}\left(t, x^{*}(t)\right)-f_{x}^{*}\left(t, x^{*}(t)\right) q^{f, q_{0}}(t)-f\left(t, x^{*}(t)\right)+f^{*}\left(t, x^{*}(t)\right) \\
&|\gamma(t)| \leq \mid 1_{M}\left[f(t, z(t))-f\left(t, x^{*}(t)\right)-f_{x}^{*}\left(t, x^{*}(t)\right) q^{f, q_{0}}(t)\right] \\
&+\left(1-1_{M}\right)\left[f^{*}(t, z(t))-f^{*}\left(t, x^{*}(t)\right)-f_{x}^{*}\left(t, x^{*}(t)\right) q^{f, q_{0}}(t)\right] \mid \\
& \leq 1_{M} 2 \beta(t)\left|q^{f, q_{0}}(t)\right|+o_{t}^{*}\left(\left|q^{f, q_{0}}(t)\right|\right) \leq 1_{M} 2 \beta(t) K^{*} d+o_{t}^{*}\left(d K^{*}\right)=: \gamma_{*}(t, d) \leq 4 \beta(t) K^{*} d
\end{aligned}
$$

for a.e. $t$. Dividing by $d$, we get

$$
|\gamma(t)| / d \leq \gamma_{*}(t, d) / d \leq 4 \beta(t) K^{*}
$$

By Lebesgue’s dominated convergence theorem, $\gamma^{*}(t, d):=\int_{0}^{t}(1 / d) \gamma_{*}(s, d) \mathrm{d} s \leq \gamma^{*}(T, d) \downarrow 0$ when $d \downarrow 0$. As

$$
\begin{aligned}
& \left|f\left(t, x^{f}(t)\right)-f^{*}\left(t, x^{*}(t)\right)-\dot{q}^{f, q_{0}}(t)\right| \\
& =\left|f\left(t, x^{f}(t)\right)-f(t, z(t))+\gamma(t)\right| \leq \beta(t)\left|x^{f}(t)-z(t)\right|+|\gamma(t)|
\end{aligned}
$$

then

$$
\begin{aligned}
\left|x^{f}(t)-x^{*}(t)-q^{f, q_{0}}(t)\right| \leq & \left|x^{f}(0)-x^{*}(0)-q_{0}\right|+d \gamma^{*}(t, d) \\
& +\int_{0}^{t} \beta(s)\left|x^{f}(s)-x^{*}(t)-q^{f, q_{0}}(t)\right| \mathrm{d} s
\end{aligned}
$$

and then by Gronwall's inequality,

$$
\left|x^{f}(t)-x^{*}(t)-q^{f, q_{0}}(t)\right| \leq\left|x^{f}(0)-x^{*}(0)-q(0)\right| \mathrm{e}^{\mathrm{j}_{0}^{t} \beta(s) \mathrm{d} s}+o(t, d)
$$

where $o(t, d)$, a second order term in $d$, equals $d\left[\gamma^{*}(t, d)+\int_{0}^{t} \gamma^{*}(s, d) \beta(s) \mathrm{e}^{\int_{s}^{t} \beta(r) \mathrm{dr}} \mathrm{d} s\right] \leq d \gamma^{*}(T, d) \mathrm{e}^{\int_{0}^{T} \beta(r) \mathrm{d} r}$.

Lemma E. In the situation of Lemma $\mathrm{B}$, let $x \rightarrow f(t, x)$ be differentiable at $\left(t, x^{*}(t)\right)$, where 
$x^{*}()=.\left(y_{*}(),. z_{*}().\right)$ is a given solution on $\left[t_{0}, \infty\right)$ of $\dot{x}=f(t, x), \quad x^{*}\left(t_{0}\right)$ given, assumed to exist. For each $d \in\left(0, d_{*}\right]$, let $x^{d}()=.\left(y^{d}(),. z^{d}().\right)$ be a solution on $\left[t_{0}, \infty\right), \quad t_{0} \geq 0$, of $\dot{x}=f(t, x), \quad x^{d}\left(t_{0}\right)$ given (it does exist), and let $q($.$) be a solution of \dot{q}=f_{x}\left(t, x^{*}(t)\right) q, q\left(t_{0}\right)$ given. Assume that, for some $K$, $\left|x^{d}\left(t_{0}\right)-x^{*}\left(t_{0}\right)-d q\left(t_{0}\right)\right| \leq o_{*}(d)$ for all $q\left(t_{0}\right) \in B(0, K), \quad o_{*}($.$) a second order term in d$. Then for some second order term $o(d), \int_{t_{0}}^{\infty}\left|\dot{y}^{d}(t)-\dot{y}_{*}(t)-d \dot{q}^{y}(t)\right| \mathrm{d} t \leq o(d)$.

Proof of Lemma E. By Lemma D, for some term $o(t, d)$ being of the second order in $d$, $\left|x^{d}(t)-x^{*}(t)-d q(t)\right| \leq o(t, d)$, when $x^{d}\left(t_{0}\right)-x^{*}\left(t_{0}\right)-d q\left(t_{0}\right)$ is of the second order. For some $d^{\prime}>0$, $\left|x^{d}\left(t_{0}\right)-x^{*}\left(t_{0}\right)\right| \leq\left|d q\left(t_{0}\right)\right|+d \leq d(K+1)$ for $d \in\left(0, d^{\prime}\right]$. Hence, by Lemma $\mathrm{B}$, for some constants $\Gamma^{*}, Q^{*}$, we have that $\left|y^{d}(t)-y_{*}(t)\right| \leq d \Gamma^{*}, \quad\left|z^{d}(t)-z_{*}(t)\right| \leq d \Gamma^{*} \mathrm{e}^{(\kappa+\omega) t}, \quad\left|q^{y}(t)\right| \leq Q^{*}, \quad\left|q^{z}(t)\right| \leq Q^{*} \mathrm{e}^{(\kappa+\omega) t}$, so

$$
\begin{aligned}
& \left|f^{y}\left(t, x^{d}(t)\right)-f^{y}\left(t, x^{*}(t)\right)-d \dot{q}^{y}(t)\right| / d \leq A^{*} \mathrm{e}^{-a t}\left|y^{d}(t)-y_{*}(t)\right| / d+B^{*} \mathrm{e}^{-b t}\left|z^{d}(t)-z_{*}(t)\right| / d \\
& +A^{*} \mathrm{e}^{-a t}\left|d q^{y}(t)\right| / d+B^{*} \mathrm{e}^{-b t}\left|d q^{z}(t)\right| / d \leq\left(A^{*}+B^{*}\right) \Gamma^{*} \mathrm{e}^{-\omega t}+\left(A^{*}+B^{*}\right) Q^{*} \mathrm{e}^{-\omega t}
\end{aligned}
$$

By Lebesgue's dominated convergence theorem the conclusion in the lemma follows if we can prove that for each $t,(1 / d)\left|f^{y}\left(t, x^{d}(t)\right)-f^{y}\left(t, x^{*}(t)\right)-d \dot{q}^{y}(t)\right| \rightarrow 0$ when $d \rightarrow 0$. To obtain the latter fact, let $z^{d}(t)=x^{*}(t)+d q(t)$, and note that

$$
\begin{aligned}
& (1 / d)\left|f^{y}\left(t, x^{d}(t)\right)-f^{y}\left(t, x^{*}(t)\right)-d \dot{q}^{y}(t)\right| \\
& \leq(1 / d)\left|f^{y}\left(t, x^{d}(t)\right)-f^{y}\left(t, z^{d}(t)\right)\right|+\left|f^{y}\left(t, z^{d}(t)\right)-f^{y}\left(t, x^{*}(t)\right)-f_{x}^{y} d q(t)\right| \\
& \leq\left\{\left(A^{*}+B^{*}\right)\left|x^{d}(t)-z^{d}(t)\right|+o_{t}^{y}(|d q(t)|)\right\} \\
& \leq(1 / d)\left\{o(t, d)+o_{t}^{y}\left(d Q^{*} \mathrm{e}^{\kappa t}\right)\right\},
\end{aligned}
$$

where the second order term $o_{t}^{y}(r)=\sup _{\left|x^{\prime}\right| \leq r}\left|f^{y}\left(t, x^{*}+x^{\prime}\right)-f^{y}\left(t, x^{*}(t)\right)-f_{x}^{y}\left(t, x^{*}(t)\right) x^{\prime}\right|$

Proof of Lemma 1.

Consider the map $z \rightarrow w(z):=\pi x^{*}(\infty)-\pi x\left(\infty, c(z), P^{*}\right)+\pi q^{*}\left(\infty, c(z), P^{*}\right)+d y$ for $z \in B(d y, d \delta), \quad d$ any number in $(0,1]$. Let $T=\max \left\{s_{i}^{*}\right\}+1$, and note that, by Lemma $C, x\left(T, c(z), P^{*}\right)$ and $q^{*}\left(T, c(z), P^{*}\right)$ are continuous in $z \in B(d y, d \delta)$. Then, by Lemma $B, \pi x\left(\infty, c(z), P^{*}\right)$ and $\pi q^{*}\left(\infty, c(z), P^{*}\right)$ are continuous in $z \in B(d y, d \delta)$. Let $C$ be a Lipschitz rank of $c(z)$ and $\lambda(z)$. For $\kappa^{*}=|y|+\delta$, let the second order term $o^{*}(d)$ satisfy

$$
\left|\pi x^{*}(\infty)-\pi x\left(\infty, c, P^{*}\right)+\pi q\left(\infty, c, P^{*}\right)\right| \leq o^{*}(d) \text {, when } c \in E_{m^{*}},|c| \leq \kappa^{*} C d,
$$

for the existence of $o^{*}(d)$ see Lemmas $\mathrm{D}$ and E. Recall that $\pi q^{*}(\infty, c, P)=\pi q^{* *}(\infty, c, P)$ and that $\left|\pi q^{* *}(\infty, c, P)-\pi q(\infty, c, P)\right| \leq o^{* *}(d)$ when $|c| \leq C \kappa^{*} d$ for some second order term $o^{* *}($.$) , see an argument$ preceding (44) and Lemma A. Hence, for $o(d)=o^{*}(d)+o^{* *}(d)$,

$\left|\pi x^{*}(\infty)-\pi x\left(\infty, c, P^{*}\right)+\pi q^{*}\left(\infty, c, P^{*}\right)\right| \leq o(d)$ when $c \in E_{m^{*}}, \quad|c| \leq \kappa^{*} C d$.

Note that for $z \in B(d y, d \delta)$, we have $w(z) \in \operatorname{cl} B(d y, o(d))$, as $|c(z)| \leq C|z| \leq \kappa^{*} C d$, and for $d$ small $(0<d \leq \rho$ for some $\rho>0), \operatorname{cl} B(d y, o(d)) \subset B(d y, d \delta)$. Fix such a $d>0$. Now, $w(z)$ is continuous in $\operatorname{cl} B(d y, o(d))$ and has a fixed point $z^{d}$ here, by Brouwer's fixed point theorem. As $w\left(z^{d}\right)=z^{d}$ and 
$z^{d}=\sum_{i} \lambda_{i}\left(z^{d}\right) q^{i}+\pi q^{*}\left(\infty, c\left(z^{d}\right), P^{*}\right), \quad 0=\pi x^{*}(\infty)-\left(\sum_{i} \lambda_{i}\left(z^{d}\right) q_{i}+\pi x\left(\infty, c\left(z^{d}\right), P^{*}\right)\right)+d y$, $\left|c\left(z^{d}\right)-c(d y)\right| \leq C\left|z^{d}-d y\right| \leq \operatorname{Co}(d), \quad\left|\lambda\left(z^{d}\right)-\lambda(d y)\right| \leq \operatorname{Co}(d)$. Then let $q^{d}=\sum_{i} \lambda_{i}\left(z^{d} / d\right) q^{i}$, $c^{d}=c\left(z^{d} / d\right)$ to obtain Lemma 1.

Observation A. On the space of continuous real-valued functions on $[0, \infty)$ with compact support, furnished with the sup-norm, $\mu_{j}$ can be represented by a nondecreasing bounded function $\mu^{j}$ such that $\int_{[0, \infty)} \phi(t) \mathrm{d} \mu^{j}(t)=\int_{[0, \infty)} \phi(t) \mathrm{d} \mu_{j}(t)$ for all bounded continuous $\phi$ with compact support. In fact, we can let $\mu^{j}(s)=\inf \left\{\mu_{j}\left(\left[0, s^{\prime}\right]\right): s^{\prime}>s\right\}, \quad \mu^{j}(0)=0,\left(\mu^{j}\right.$ right continuous for $\left.s>0\right)$. Let $J_{*}$ be the continuity points in $(0, \infty)$ of $s \rightarrow \int_{[0, s]} \mathrm{d} \mu_{j}$. Then $\mu_{j}([0, s]) \leq \mu^{j}(s)=: \int_{[0, s]} \mathrm{d} \mu^{j}(t)=: \mu^{j}([0, s]) \leq \mu_{j}\left(\left[0, s^{\prime}\right]\right)$ for any $s^{\prime}>s$, hence for $s \in J_{*}, \quad \mu_{j}([0, s])=\mu^{j}([0, s])$ and $\sup _{s^{\prime \prime}<s} \mu^{j}\left(\left[0, s^{\prime \prime}\right]\right)=\sup _{s^{\prime \prime}<s, s^{\prime \prime} \in J_{*}} \mu^{j}\left(\left[0, s^{\prime \prime}\right]\right)=\sup _{s^{\prime \prime}<s, s^{\prime \prime} \in J_{*}} \mu_{j}\left(\left[0, s^{\prime \prime}\right]\right)=\mu_{j}([0, s])=\mu^{j}([0, s]), \quad$ so $\quad \mu^{j} \quad$ is also left continuous at $s$. For piecewise constant functions $\phi$ with bounded support, jumping only at points in $J_{*}$, evidently $\int_{[0, \infty)} \phi(t) \mathrm{d} \mu^{j}(t)=\int_{[0, \infty)} \phi(t) \mathrm{d} \mu_{j}(t)$. By approximating continuous function (or even piecewise continuous functions jumping only at points in $J_{*}$ ) by such piecewise constant functions, one see that the same equality holds for continuous functions (or such piecewise continuous functions) $\phi$ with bounded support.

Note that if $\phi_{n} \rightarrow \phi$ uniformly ( $\phi_{n}, \phi$ continuous, with a common bounded support), and $s_{n} \rightarrow s \in J_{*}$, then $\int_{\left[s_{n}, \infty\right)} \phi_{n}(t) \mathrm{d} \mu_{j}(t) \rightarrow \int_{[s, \infty)} \phi(t) \mathrm{d} \mu_{j}(t)$ : Assuming for simplicity (say) $s_{n} \downarrow s$, this follows from

$$
\begin{aligned}
\left|\int_{\left[s_{n}, \infty\right)} \phi_{n}(t) \mathrm{d} \mu_{j}(t)-\int_{[s, \infty)} \phi(t) \mathrm{d} \mu_{j}(t)\right| & =\left|\int_{\left[s_{n}, \infty\right)} \phi_{n}(t) \mathrm{d} \mu_{j}(t)-\int_{\left[s_{n}, \infty\right)} \phi(t) \mathrm{d} \mu_{j}(t)+\int_{\left[s_{n}, \infty\right)} \phi(t) \mathrm{d} \mu_{j}(t)-\int_{[s, \infty)} \phi(t) \mathrm{d} \mu_{j}(t)\right| \\
& \leq\left|\phi_{n}-\phi\right|_{\infty} \mu_{j}([0, \infty))+|\phi|_{\infty} \mu_{j}\left(\left[s, s_{n}\right)\right) .
\end{aligned}
$$

Let $\varepsilon>0, \quad \varepsilon$ arbitrary. For $T>T^{*}, T^{*}$ large, $0 \leq \mu_{j}([T, \infty))-\mu_{j}(\infty) \leq \varepsilon$, hence, for any $S>T>T^{*}$, $\mu_{j}([T, S)) \leq 2 \varepsilon$. Thus for $T \in J_{*}, T>T^{*}$,

$2 \varepsilon \geq \sup _{S} \mu_{j}([T, S))=\sup _{S \in J_{*}} \mu_{j}([T, S))=\sup _{S} \mu^{j}([T, S))=: \mu^{j}([T, \infty))=: \int_{[T, \infty)} \mathrm{d} \mu^{j}$. Hence, $\mu^{j}([T, \infty)) \rightarrow 0$, when $T \rightarrow \infty$. If $\phi$ is bounded and continuous, but with unbounded support, evidently, by the last inequality, $\left|\int_{[s, S]} \phi(t) \mathrm{d} \mu_{j}(t)-\int_{[s, T]} \phi(t) \mathrm{d} \mu_{j}(t)\right| \leq \int_{(T, S+1)}|\phi|_{\infty} \mathrm{d} \mu_{j}(t) \leq|\phi|_{\infty} 2 \varepsilon$, so $\lim _{T \rightarrow \infty} \int_{[s, T]} \phi \mathrm{d} \mu_{j}$ exists. As also $\mu^{j}([T, S)) \leq 2 \varepsilon \quad\left(\mu^{j}([T, S)) \leq \mu_{j}\left(\left[T^{\prime}, S^{\prime}\right)\right), T^{*}<T^{\prime}<T, S<S^{\prime}\right)$, exactly the same argument works for $\lim _{T \rightarrow \infty} \int_{[s, T]} \phi \mathrm{d} \mu^{j}$, the latter limit written $\int_{[s, \infty)} \phi \mathrm{d} \mu^{j}$.

$$
\text { Proof of }(10)(\gamma) \Rightarrow\left(p_{\infty}, \lim _{s \rightarrow 0} \sum_{j \in G(0+)} \mu_{j}([s, \infty))\right) \neq 0 \text {. }
$$

Assume again that $a x(\infty)$ is maximized and postulate the conditions in Theorem 2 (allow even for the conditions in Remark 3), in particular postulate (10) ( $\gamma$ ). For simplicity, assume $a_{j}=0$ for $j \leq j^{\prime}, a_{j} \geq 2$ for $j>j^{\prime}, \quad \tilde{k}=2$. We want to replace each condition $g^{j} \geq 0, j \leq j^{\prime}$ by two conditions, $g^{j} \geq 0$ for $t \leq 2$, $g^{j} \geq 0$ for $t \geq 2$. It can be done by requiring that $g^{j} \geq 0, j \leq j^{\prime}$ holds for $t \leq 2$ and, by adding new constraints $g^{j *+j} \geq 0$ required to hold on $[2, \infty)$, where $g^{j *+j}=g^{j}$ for $j \leq j^{\prime}$. We now first assume both that $g^{j}, j \leq j^{\prime}$ is independent of $t$, and that $f$ is independent of $t$ for $t \in[0,2]$. Assume that there exist $v_{i} \in U$, $i=1, \cdots, i^{*}, \quad \lambda_{i}>0, \quad \sum \lambda_{i}=1$ (see (9)) such that

$$
7 \beta^{*}:=\min _{j \in G(0+)} g_{x}^{j}\left(0, x_{0}\right) \sum_{i} \lambda_{i} f\left(0, x_{0}, v_{i}\right)>0
$$


Then, for some $\delta>0, \varepsilon \in(0,1]$, both

$$
j \notin G(0+) \Rightarrow g^{j}\left(0, x^{*}(t)\right)>0 \text { for } t \in(0,2 \varepsilon)
$$

and, for $0 \leq s \leq t \leq \varepsilon$,

$$
\min _{j \in G(0+), x \in B\left(x_{0}, \delta\right)} g_{x}^{j}(0, x) C(t, s) \sum_{i} \lambda_{i} f\left(0, x, v_{i}\right) \geq 6 \beta^{*}
$$

Let $M$ have the property that $\max _{i}\left|f\left(0, x, v_{i}\right)\right| \leq M$ and $\left|f\left(0, x, u^{*}(t)\right)\right| \leq M$ for $x \in B\left(x_{0}, \delta\right)$. Let $O(\breve{\gamma})$ be a first order term (i.e. $O(\breve{\gamma}) \downarrow 0$ when $\breve{\gamma} \downarrow 0$ ), such that $O(\breve{\gamma}) \geq\left|g_{x}^{j}(0, x) f\left(0, x, v_{i}\right)-g_{x}^{j}\left(0, x^{\prime}\right) f\left(0, x^{\prime}, v_{i}\right)\right|$ when $x, x^{\prime} \in B\left(x_{0}, \breve{\gamma}\right)$ for all $j \leq j^{\prime}, i$. As we did in connection with (43), let $a_{0}=1 / 2$ and define inductively $a_{j}=a_{j-1}-\left(a_{j-1}\right)^{2} \quad\left(a_{j} \downarrow 0\right)$. Let $A_{j}=\left[a_{j}, a_{j-1}\right)$. Choose a partition $M_{j}^{i}=\left[a_{j}^{i}, b_{j}^{i}\right), \quad i=1, \cdots, i^{*}$, of $A_{j}$ such that $\left(b_{j}^{i}-a_{j}^{i}\right)=\lambda_{i}$ meas $\left(A_{j}\right)$. Define $\bar{u}(t)=\sum_{i, j} v_{i} 1_{M_{j}^{i}}(t)$. It is easily seen that for $t \in(0,1 / 2)$,

$$
\left|\int_{0}^{t} f(0, x, \bar{u}(t)) \mathrm{d} t-t \sum_{i} \lambda_{i} f\left(0, x, v_{i}\right)\right| \leq 8 t^{2} M
$$

for any $x \in B\left(x_{0}, \delta\right)$. To see this, note that for $t=a_{j}$ the left hand side vanishes, while for $t \in\left(a_{j}, a_{j-1}\right)$ $\left(\Rightarrow t \geq a_{j-1}\left(1-a_{j-1}\right) \geq a_{j-1} / 2\right)$, the left hand side is smaller that $2\left(t-a_{j}\right) M \leq 2\left(a_{j-1}-a_{j}\right) M=2 a_{j-1}^{2} M \leq 8 t^{2} M$. Let $j^{\prime \prime}=j^{*}+j^{\prime}$. Define $\Gamma:=\max _{t \geq s \geq 0}\left|g_{x}\left(t, x^{*}(t)\right) C(t, s)\right|$, $M^{\prime \prime}:=\sup _{t \geq s \geq 0}|\pi C(t, s)|, \quad \Theta:=\max _{j}\left|g_{x}^{j}\left(0, x_{0}\right)\right|$. Let $\alpha \in(0, \delta / 2 M]$ be any given number, $\alpha \leq \varepsilon / 2$, $\alpha \leq \beta^{*} / M\left(2 \Gamma+M^{\prime \prime}\right)$, such that $O(M \alpha) \leq \beta^{*}$ and $\left(\Theta+2 \Gamma+M^{\prime \prime}\right) 8 t^{2} M \leq t \beta^{*}$ when $t \in[0, \alpha]$.

Let $\tau \in(0, \varepsilon / 2], \quad \tau<\delta / 2 M$. Moreover, let $\bar{x}(t)=x^{*}(\tau)+\int_{\tau}^{t} f(0, \bar{x}(r), \bar{u}(r)) \mathrm{d} r, \quad t \in(\tau, 2]$. Then $\left|\bar{x}(t)-x^{*}(\tau)\right| \leq M \hat{\gamma}$ when $t-\tau \in[0, \hat{\gamma}], \quad \hat{\gamma} \leq \min \{1 / 2, \delta / 2 M\}$, as $x^{*}(\tau) \in B\left(x_{0}, \delta / 2\right)$. So $\left|g_{x}^{j}(0, \bar{x}(t)) f(0, \bar{x}(t), \bar{u}(t))-g_{x}^{j}\left(0, x^{*}(\tau)\right) f\left(0, x^{*}(\tau), \bar{u}(t)\right)\right| \leq O(M \hat{\gamma})$ when $|t-\tau| \leq \hat{\gamma}$.

Consider the following auxiliary control problem on $[-1-\alpha+\tau, \infty)$. Define $\tilde{u}(t)=\bar{u}(t+\alpha-\tau)$, $t \in[-\alpha+\tau, \tau] . \quad$ Let $\quad \tilde{f}=y f(0, x, \tilde{u}(t)) 1_{[-\alpha+\tau, \tau)}(t)+(1-\alpha y) f(0, x, u) 1_{[\tau, 1+\tau)}(t)+f(t, x, u) 1_{[1+\tau, \infty)}(t)$, and introduce the two state equations $\dot{x}=\tilde{f}, \quad x(-1-\alpha+\tau)=x^{*}(\tau), \quad \dot{y}=w 1_{[-1-\alpha+\tau,-\alpha+\tau)}, \quad y(-1-\alpha+\tau)=0, \quad y(\infty)$ free, and let $u \in U$ and $w \geq 0$ be the controls. We require $g^{j}(t, x) \geq 0$ on $[\tau, 2]$ for $j \leq j^{\prime}$ and $g^{j}(t, x) \geq 0$ on $[2, \infty)$ for $j>j^{\prime}$, and $y(t) \leq \xi>0, \xi$ described below. The end conditions on $x(\infty)$ are as before. Then $y \equiv w \equiv 0$ and $u()=.u^{*}$.) (on $[\tau, \infty)$ ) are optimal in this problem, see below. Applying Theorem 2 to this problem, with $p$ and $p_{y}$ as costates corresponding to $x$ and $y$, gives $\mu_{j} \geq 0$, $\mu_{j}([-1-\alpha+\tau, \tau))=0, \quad \mu_{j}\left([\tau, \infty) \cap \complement J^{*}(j, \beta)\right)=0$ for all $\beta>0, \quad \mu_{j}([\tau, 2))=0$ for $j>j^{\prime}$, $\mu_{j}((2, \infty))=0, \quad j \leq j^{\prime}$, and the maximum condition (22), i.e. (a.e.)

$$
p(s)\left(f\left(s, x^{*}(s), u\right)-f^{*}(s)\right) \leq 0, \quad s \geq \tau, \quad p_{y}(s) w \leq 0, \quad s \in[-1-\alpha+\tau,-\alpha+\tau],
$$

where

$$
\begin{gathered}
p(s)=p_{\infty} C(\infty, s)+\sum_{j \int_{[s, \infty)}} \hat{g}_{x}^{j}(t) C(t, s) \mathrm{d} \mu_{j}(t), \\
\dot{p}_{y}(t)=-p(t) f\left(0, x^{*}(\tau), \tilde{u}(t)\right) 1_{[-\alpha+\tau, \tau]}(t)+\alpha p(t) f^{*}(t) 1_{[\tau, 1+\tau)}(t),
\end{gathered}
$$


$p_{y}(\infty)=p_{y}(1+\tau)=0$. In particular, because $\inf _{t \in[\tau, \tau+\varepsilon]} g^{j}\left(0, x^{*}(t)\right)>0$ when $j \notin G(0+), \quad j \leq j^{\prime}$, see (72), then

$$
j \notin G(0+) \Rightarrow \mu_{j}([\tau, \tau+\varepsilon])=0 .
$$

Assume by contradiction both that $\sum_{j \leq j^{*}+j^{\prime}} \mu_{j}([\alpha+\tau, \infty)) \leq \gamma \alpha \leq \gamma$ and that $\left|p_{\infty}\right| \leq \gamma \alpha$, where $\gamma:=\sum_{j \leq j^{*}+j^{\prime}} \mu_{j}([\tau, \alpha+\tau))=\sum_{j \in G(0+)} \mu_{j}([\tau, \alpha+\tau))$, which by necessity means that $\gamma>0$. Then, fo $\mathrm{r}$

$$
\begin{gathered}
\kappa(s):=\sum_{j \in G(0+)} \int_{[s, \alpha+\tau)} g_{x}^{j}\left(0, x^{*}(r)\right) C(r, s) \mathrm{d} \mu_{j}(r), \\
\kappa^{*}(s):=\int_{[s, \infty)} \sum_{j} g_{x}^{j}\left(r, x^{*}(r)\right) C(r, s) \mathrm{d} \mu_{j}(r),
\end{gathered}
$$

$p(s)=p_{\infty} C(\infty, s)+\kappa^{*}(s)$ for $s \geq \tau+\alpha, \quad p(s)=p_{\infty} C(\infty, s)+\kappa(s)+\kappa^{*}(\tau+\alpha)$ for $s \in[\tau, \alpha+\tau)$, $p(s)=p(\tau)$ for $s<\tau$, which gives $\left|\kappa^{*}(s)\right| \leq \alpha \gamma \Gamma$ and $|p(s)| \leq \alpha \gamma\left(\Gamma+M^{\prime \prime}\right)$ for $s \geq \alpha+\tau$, and $|p(s)| \leq \gamma \Gamma+\alpha \gamma\left(\Gamma+M^{\prime \prime}\right) \leq \gamma\left(2 \Gamma+M^{\prime \prime}\right)$ for $s \in[\tau, \tau+\alpha]$. Also, $\kappa(\tau) \alpha \sum_{i} \lambda_{i} f\left(0, x^{*}(\tau), v_{i}\right) \geq 6 \alpha \beta^{*} \gamma$, by (73). As $p_{y}(\tau+1)=p_{y}(\infty)=0$, using (74), we have, for $t \in[-2 \alpha+\tau,-\alpha+\tau]$,

$$
\begin{aligned}
p_{y}(t)= & p_{y}(\tau+1)+\int_{-\alpha+\tau}^{\tau} p(\tau) f\left(0, x^{*}(\tau), \tilde{u}(t)\right) \mathrm{d} t-\int_{\tau}^{\tau+1} \alpha p(t) f\left(0, x^{*}(t), u^{*}(t)\right) \mathrm{d} t \\
= & p(\tau)\left[\int_{-\alpha+\tau}^{\tau} f\left(0, x^{*}(\tau), \tilde{u}(t)\right) \mathrm{d} t-\alpha \sum_{i} \lambda_{i} f\left(0, x^{*}(\tau), v_{i}\right)\right] \\
& +\left[p_{\infty} C(\infty, \tau)+\kappa^{*}(\tau+\alpha)\right] \alpha \sum_{i} \lambda_{i} f\left(0, x^{*}(\tau), v_{i}\right)+\kappa(\tau) \alpha \sum_{i} \lambda_{i} f\left(0, x^{*}(\tau), v_{i}\right) \\
& -\int_{\tau}^{\tau+\alpha} \alpha p(t) f\left(0, x^{*}(t), u^{*}(t)\right) \mathrm{d} t-\int_{\tau+\alpha}^{\tau+1} \alpha p(t) f\left(0, x^{*}(t) u^{*}(t)\right) \mathrm{d} t \\
\geq & -\gamma\left(2 \Gamma+M^{\prime \prime}\right) 8 \alpha^{2} M-\alpha M\left|p_{\infty}\right||\pi C(\infty, \tau)|-\alpha M\left|\kappa^{*}(\tau+\alpha)\right|+6 \alpha \beta^{*} \gamma \\
& -\int_{\tau}^{\tau+\alpha} \alpha \gamma\left(2 \Gamma+M^{\prime \prime}\right) M \mathrm{~d} t-\int_{\tau+\alpha}^{\tau+1} \alpha^{2} \gamma\left(\Gamma+M^{\prime \prime}\right) M \mathrm{~d} t \\
\geq & -\alpha \gamma \beta^{*}-\alpha \gamma \beta^{*}-\alpha \gamma \beta^{*}+6 \alpha \gamma \beta^{*}-\alpha \gamma \beta^{*}-\alpha \gamma \beta^{*} \geq \alpha \gamma \beta^{*}>0,
\end{aligned}
$$

contradicting $p_{y}(s) w \leq 0$, for $s \in(-2 \alpha+\tau, \alpha+\tau)$ (as $w \equiv 0$ is optimal).

The optimality of $w \equiv 0, x^{*}(),. u^{*}($.$) follows from the following argument: Let x(),. y(),. u(),. w($.$) be an$ arbitrary admissible quadruple in the auxiliary problem. Let $y=y(-\alpha+\tau)$. If $y>0$, let $x^{y}(t)=$ $x^{y}(t)=x(\tau+(t-\tau) / y), \quad u^{y}(t)=\tilde{u}(\tau+(t-\tau) / y)$ for $t \in[-\alpha y+\tau, \tau)$, let $x^{y}(t)=x(\tau+(t-\tau) /(1-\alpha y))$, $u^{y}(t)=u(\tau+(t-\tau) /(1-\alpha y))$ for $t \in[\tau, 1-\alpha y+\tau)$, and let $x^{y}(t)=x(t+\alpha y), \quad u^{y}(t)=u(t+\alpha y)$ for $t \geq 1-\alpha y+\tau$. Next, let $x_{y}(t)=x^{y}(t-\alpha y), \quad u_{y}(t)=u^{y}(t-\alpha y), \quad t \in[\tau, \infty)$. Then

$$
\begin{aligned}
\dot{x}^{y}(t)= & \dot{x}(\tau+(t-\tau) / y) / y 1_{[-\alpha y+\tau, \tau)}(t) \\
& +\dot{x}(\tau+(t-\tau) /(1-\alpha y)) /(1-\alpha y) 1_{[\tau, 1-\alpha y+\tau)}(t)+\dot{x}(t+\alpha y) 1_{[1-\alpha y+\tau, \infty)}(t) \\
= & f\left(0, x^{y}(t), u^{y}(t)\right) 1_{[-\alpha y+\tau, 1-\alpha y+\tau]}(t)+f\left(t+\alpha y, x^{y}(t), u^{y}(t)\right) 1_{[1-\alpha y+\tau, \infty)}(t)
\end{aligned}
$$

and hence $\dot{x}_{y}(t)=f\left(t, x_{y}(t), u_{y}(t)\right)$. Moreover, $x_{y}(\tau)=x^{*}(\tau)$.

So $x_{y}(t), \quad y>0$, is a solution in the original system evidently satisfying the end restrictions, and $0 \leq g^{j}(t, x(t))=g^{j}\left(t, x_{y}(t)\right)$ for $t \geq 2, \quad j>j^{\prime}$. Because $g^{j}(0, x(t)) \geq 0, \quad t \in[\tau, 2]$ for $j \leq j^{\prime}$, $g^{j}\left(0, x^{y}(t)\right) \geq 0, \quad t \in[\tau, 2-\alpha y]$, and then $g^{j}\left(t, x_{y}(t)\right) \geq 0$ for $t \in[\tau+\alpha y, 2], \quad j \leq j^{\prime}$, Finally, on $[\tau-\alpha y, \tau] \quad g^{j}\left(0, x^{y}(t)\right) \geq 0, j \leq j^{\prime}$ as we shall see in a moment, so $g^{j}\left(0, x_{y}(t)\right) \geq 0$ for $t \in[\tau, \alpha y+\tau)$, hence $g^{j}\left(t, x_{y}(t)\right) \geq 0$ for all $t \in[\tau, 2], j \leq j^{\prime}$. For $y=0$, let $x^{y}(t)=x_{y}(t)=x(t)$, in which case $x_{y}($. is automatically an original solution.

From (74) and $y \int_{-\alpha+\tau}^{-\alpha+\tau+t} f\left(0, x^{*}(\tau), \tilde{u}(s)\right) \mathrm{d} s=\int_{-\alpha y+\tau}^{(-\alpha+t) y+\tau} f\left(0, x^{*}(\tau), u^{y}(\hat{t})\right) \mathrm{d} \hat{t}$, for $t \in(0, \alpha]$, we get 


$$
\left|\int_{-\alpha y+\tau}^{(-\alpha+t) y+\tau} f\left(0, x^{*}(\tau), u^{y}(\hat{t})\right) \mathrm{d} \hat{t}-t y \sum_{i} \lambda_{i} f\left(0, x^{*}(\tau), v_{i}\right)\right| \leq y 8 t^{2} M
$$

Using $x^{y}(-\alpha y+\tau)=x^{*}(\tau)$, when $t \in[0, \alpha]$, for $j \leq j^{\prime}$,

$$
\begin{aligned}
& g^{j}\left(0, x^{y}(-\alpha y+t y+\tau)\right)-g^{j}\left(0, x^{*}(\tau)\right) \\
& =\int_{-\alpha y+\tau}^{(-\alpha+t) y+\tau} g_{x}^{j}\left(0, x^{y}(r)\right) f\left(0, x^{y}(r), u^{y}(r)\right) \mathrm{d} r \\
& \geq \int_{-\alpha y+\tau}^{(-\alpha+t) y+\tau} g_{x}^{j}\left(0, x^{*}(\tau)\right) f\left(0, x^{*}(\tau), u^{y}(r)\right) \mathrm{d} r-\operatorname{ty} O(M \alpha) .
\end{aligned}
$$

Then, by (72), (73), (76), for all $y \in(0,1]$, all $t \in[0, \alpha]$, all $j \in G(\tau)$,

$$
\begin{aligned}
& g^{j}\left(0, x^{y}(-\alpha y+t y+\tau)\right)-g^{j}\left(0, x^{*}(\tau)\right) \\
& \geq-\operatorname{ty} O(M \alpha)+\int_{-\alpha y+\tau}^{(-\alpha+t) y+\tau} g_{x}^{j}\left(0, x^{*}(\tau)\right) f\left(0, x^{*}(\tau), u^{y}(r)\right) \mathrm{d} r \\
& \geq-\operatorname{ty} O(M \alpha)+t y g_{x}^{j}\left(0, x^{*}(\tau)\right) \sum_{i} \lambda_{i} f\left(0, x^{*}(\tau), v_{i}\right)-y \Theta 8 t^{2} M \\
& \geq \operatorname{ty}\left(6 \beta^{*}-\beta^{*}-\beta^{*}\right) \geq 0 .
\end{aligned}
$$

As $g^{j}\left(0, x^{*}(\tau)\right) \geq 0$, then $g^{j}\left(0, x^{y}((-\alpha+t) y+\tau)\right) \geq 0 \quad$ for $t \in[0, \alpha], \quad j \in G(\tau)$. Moreover, for $\xi^{*}=\min _{j \notin G(\tau)} g^{j}\left(0, x^{*}(\tau)\right)>0$, for some positive $\xi$ small enough, $g^{j}\left(0, x^{y}((-\alpha+t) y+\tau)\right) \geq 0$ when $y \leq \xi, \quad t \in[0, \alpha]$. (For $y=0$ there is nothing to prove.)

Now, $\pi x_{y}(t)=\pi x(t)$ for $t \geq 2$, so $\left(x_{y}(),. u_{y}().\right)$ belongs to the set of admissible pairs in the original problem. We have $a x(\infty)=a x_{y}(\infty) \leq a x^{*}(\infty)$, hence $w(.) \equiv 0, u^{*}($.$) are optimal in the auxiliary problem { }^{19}$.

Let now $\tau=\tau^{n} \in(0, \varepsilon / 2], \quad \tau^{n}<\delta / 2 M$, where $\tau^{n} \downarrow 0$, and let $p_{\infty}^{n}$ and $\mu_{j}^{n}$ be corresponding multipliers, satisfying the normalization $\left|p_{\infty}^{n}\right|+\sum_{j} \mu_{j}^{n}\left(\left[\tau_{n}, \infty\right)\right)=1$. We put $\mu_{j}^{n}\left(\left[0, \tau_{n}\right)\right)=0$. Now, $\left(p_{\infty}^{n}, \mu^{n}\right)$ has a cluster point $\left(p_{\infty}, \mu\right)$ satisfying (22), (55), and (56). Assume now that $\left(p_{\infty}, \mu([\alpha, \infty))\right)=0$. Then there exist some $n$ such that $\left|p_{\infty}^{n}\right| \leq \alpha / 4 \leq 1 / 4, \quad \sum_{j} \mu_{j}^{n}\left(\left[\tau_{n}+\alpha, \infty\right)\right) \leq \alpha / 4 \leq 1 / 4$, and hence $\gamma=\sum_{j} \mu_{j}^{n}\left(\left[\tau_{n}, \tau_{n}+\alpha\right)\right)=\sum_{j \in G(0+)} \mu_{j}^{n}\left(\left[\tau_{n}, \tau_{n}+\alpha\right)\right) \geq 1 / 2$. Hence, $\alpha / 4 \leq \alpha \gamma$, which leads to a contradiction, as was shown above. Thus, $\left(p_{\infty}, \mu([\alpha, \infty))\right) \neq 0$.

We can extend this result to problems that are nonautonomous on [0,2], by using $t$ as a new state variable, say $z$, governed by $\dot{z}=1$, with $x$ governed by $\dot{x}=\breve{f}(t, x, z, u)=f(z, x, u) 1_{[0,2)}(t)+f(t, x, u) 1_{[2, \infty)}(t)$, provided that $f(t, x, u)$ is jointly differentiable in $(t, x)$ at $\left(t, x^{*}(t)\right)$ for all $t$, and that $g^{j}(t, x)$ is differentiable in $(t, x)$ at $\left(t, x^{*}(t)\right)$ uniformly in $t \in[0,2]$, with a derivative at this point bounded uniformly in $t \in[0,2]$.

\footnotetext{
${ }^{19}$ In case we have constraints $g^{j}(t, x, u) \geq 0, \quad j>j^{*}$, (in which case $g^{j}(t, x(t), u(t)) \geq 0, \quad t \geq 0$ is required), then for $\tau$ and $\alpha$ small, for $s \in[\tau, \tau+y \alpha), g^{j}\left(t, x_{y}(s), u_{y}(s)\right) \geq \min _{i} g^{j}\left(t, x_{y}(s), v_{i}\right) \min _{i} g^{j}\left(t, x_{0}, v_{i}\right)>0$, as $v_{i} \in U(0)$.
} 